The Origin of the Information System in the RNA/Protein World: A Simulation Model of the Evolution of Translation and the Genetic Code

\author{
Sankar Chatterjee \\ Department of Geosciences \\ Museum of Texas Tech University, Box 43191 \\ Lubbock, Texas 79409, USA \\ Surya Yadav \\ Rawls College of Business, Box 42101, \\ 703 Flint Avenue, Texas Tech University, Lubbock, Texas 79409, USA
}




\section{CONTENTS}

$\underline{\text { Chapters }} \quad \underline{\text { Page }}$

$\begin{array}{ll}\text { Abstract } & 4\end{array}$

1. Introduction and Background 5

2. The Emergence of Macromolecules in the Hydrothermal Crater-Lakes $\quad 17$

2.1. Cell Membranes 18

2.2. The Selection of Homochirality 19

2.3. Polymerization 20

2.4. RNA/Protein World 20

3. The Age of Information 23

4. Temporal Order of the Emergence of the Translation machinery Systems 26

4.1. Translation Machinery Complex $\quad 27$

4.2. Selection of Amino Acids

4.3. The Origin of RNA 33

4.4. The Origin of Ribozyme 36

4.5. The Origin of Transfer RNA 39

4.6. The Origin of Aminoacyl-tRNA Synthetases 44

4.7. The Origin of Messenger RNA and Translation 48

4.8. The Origin of Ribosomes $\quad 54$

4.9. Protein Synthesis 63

5. The Origin and Evolution of the Genetic Code 67

5.1. Origin of the Genetic Code 71

5.2. Early Stage of Code Evolution: GNC Code 76

5.3. Transitional Stage of Code Evolution: SNS Code 77

5.4. Final Stage of Code Evolution: Universal of genetic Code 78

6. Coevolution of the Translation Machines and the Genetic Code 81

$\begin{array}{lr}\text { 6.1. Biological Information Systems } & 85\end{array}$

6.2. Evolution of Biological Information System $\quad 88$

6.2. Pre-tRNA/pre-aaRS/pre-mRNA Translation Machine 92

6.3. tRNA/aaRS/mRNA Translation Machine 93

6.4. tRNA/aaRS/mRNA/ribosome Translation Machine 94

7. Design of Translation Machines and the Genetic Code:
A Model-View-Controller Architecture

7.2. The Logic of the Genetic Translation Machines 96

7.3. Simulation of Translation Machines and Cells 98

7.4. Reading the Message of $m R N A \quad 100$

7.5. Genetic Code Vs. Binary Code 103 
7.6. Conversion of Three Letter Codons into Numerical Codons 105

7.7. Algorithmic Design of CATI 109

8. Simulation and Visualization of the Translation Machinery Pathways $\quad 110$

8.1. Stage I. Visualization-pre-aaRS-pre-tRNA-pre-mRNA Machine Complex 111

8.2. Stage II. Visualization-aaRS-tRNA-mRNA Machine Complex 113

8.3. Visualization-Stage III. aaRS-tRNA-mRNA-Ribosome Machine Complex 114

9. Discussion and Conclusion 116

$\begin{array}{lr}\text { Acknowledgments } & 120\end{array}$

$\begin{array}{lr}\text { References } & 121\end{array}$

$\begin{array}{lr}\text { Figure Captions } & 132\end{array}$

$\begin{array}{ll}\text { Supplemental Materials } & 141\end{array}$ 


\title{
The Origin of Information System in the RNA/Protein World: Simulation Model of the Evolution of Translation and the Genetic Code
}

\author{
Sankar Chatterjee ${ }^{1, *}$ and Surya Yadav $^{2}$ \\ 1 Department of Geosciences, Museum of Texas Tech University, Box 43191, $33014^{\text {th }}$ \\ Street, Lubbock, Texas 79409, USA \\ 2 Rawls College of Business, Box 42101, 703 Flint Avenue, Texas Tech University, \\ Lubbock, Texas 79409, USA \\ Correspondence: sankar.chatterjee@,ttu.edu; Tel: +1-806-787-4332
}

\begin{abstract}
The Late Heavy Bombardment Period (4.1 to 3.8 billion years ago) of heightened impact cratering activity on young Earth is likely the driving force for the origin of life. During the Eoarchean, asteroids such as carbonaceous chondrites delivered the building blocks of life and water to early Earth. Asteroid collisions created innumerable hydrothermal crater lakes in the Eoarchean crust which inadvertently became the perfect cradle for prebiotic chemistry. These hydrothermal crater lakes were filled with cosmic water and the building blocks of life. forming a thick prebiotic soup. The unique combination of exogenous delivery of extraterrestrial building blocks of life, and the endogenous biosynthesis in hydrothermal impact crater lakes very likely gave rise to life. A new symbiotic model for the origin of life within the hydrothermal crater lakes is here proposed. In this scenario, life arose around four billion years ago through five hierarchical stages of increasing molecular complexity: cosmic, geologic, chemical, information, and biological. During the prebiotic synthesis, membranes first appeared in the hydrothermal crater lakes, followed by the simultaneous origin of RNA and protein molecules, creating the RNA/protein world. These proteins were noncoded protein enzymes that facilitated chemical reactions. RNA molecules formed in the hydrothermal crater basin by polymerization of the nucleotides on the montmorillonite mineral substrate. Similarly, the initial synthesis of abiotic protein enzymes was mediated by the condensation of amino acids on pyrite surfaces. The regular wet-dry cycles within the crater lakes assisted further concentration, condensation, and polymerization of the RNAs and proteins. Lipid membranes randomly encapsulated amino acids, RNA, and protein molecules from the prebiotic soup to initiate a molecular symbiosis inside the protocells, this led to the hierarchical emergence of several cell components. As the role of protein enzymes became essential for catalytic process in the RNA/protein world, Darwinian selection from noncoded to coded protein synthesis led to translation systems and the genetic code, heralding the information stage. In this stage, the biochemical pathways suggest the successive emergence of translation machineries such as tRNAs, aaRS, mRNAs, and of ribosomes for protein synthesis. The molecular attraction between tRNA and amino acid led to the emergence of translation machinery and the genetic code. tRNA is an ancient molecule that created mRNA for the purpose of storing amino acid information like a digital strip. Each mRNA strand became the storage device for genetic information that encoded the amino acid sequences in triplet nucleotides. As information became available in the digital languages of the codon within mRNA, biosynthesis became less random and more organized and directional. The original translation machinery was simpler before the emergence of the ribosome than that of today. We review three main concepts on the origin and evolution of the genetic code: the
\end{abstract}


stereochemical theory, the coevolution theory, and adaptive theory. We believe that these three theories are not mutually exclusive, but are compatible with our coevolution model of translations machines and the genetic code. We suggest biosynthetic pathways as the origin of the translation machine that provided the framework for the origin of the genetic code. During translation, the genetic code developed in three stages coincident with the refinement of the translation machinery: GNC code with four codons and four amino acids during interactions of pre-tRNA/pre-aaRS /pre-mRNA, SNS code consisting of 16 codons and 10 amino acids appeared during the tRNA/aaRS/mRNA interaction, and finally the universal genetic code evolved with the emergence of the tRNA/aaRS/mRNA/ribosome machine. The universal code consists of 64 codons and 20 amino acids, with a redundancy that minimizes errors in translation. To address the question of the origin of the biological information system in the RNA/protein world, we converted letter codons into numerical codons in the Universal Genetic Code Table. We developed a software called CATI (Codon-Amino Acid-Translator-Imitator) to translate randomly chosen numerical codons into corresponding amino acids and vice versa, gaining insight into how translation might have worked in the RNA/protein world. We simulated the likely biochemical pathways for the origin of translation and the genetic code using the ModelView-Controller (MVC) software framework, and the translation machinery step-by-step. We used AnyLogic software to simulate and visualize the evolution of the translation machines and the genetic code. We conclude that the emergence of the information age from the RNA/protein world was a watershed event in the origin of life about four billion years ago.

Keywords: hierarchical origin of life; RNA/protein world; biological information system; translation and the genetic code; coevolution of translation machine and the genetic code; MVC architecture pattern and biological information; AnyLogic software for computer simulation of translation machine

\section{Introduction and Background}

The origin of life has fascinated humanity for millennia and is one of the great unsolved scientific problems of our age. Recent evidence suggests that life may have emerged on young Earth about four billion years ago through the spontaneous interaction of biomolecules in a steaming hydrothermal environment, but the actual pathways of biogenesis are still shrouded in mystery. The exquisitely intricate biochemical machinery of cells hints at a long evolutionary process. Through a historical reconstruction of the early Earth environment, we present a comprehensive theory of the origin of life, unveiling new and revised biochemical steps that describe the emergence of an extraordinary information system in the prebiotic world. 
We have used a bottom-up biogenetic approach for reconstructing the origin of life from simple chemicals to more complex. The emergence of the first cells was the culmination of a long history of prior cosmic, geologic, chemical and information processes. The earliest history, called the Hadean Eon $(\sim 4.6$ to $4 \mathrm{Ga})$ is barely recorded in the geologic record because asteroids were continuously battering the early Earth. The heat generated from those intense impacts led to large-scale melting of the Earth surface, forming a vast molten globe that obliterated most traces of early history [1]. During the beginning of the Eoarchean, as the frequency of impacts slowed down, the molten Earth cooled, clouds formed, and the crust hardened, blanketing the entire planet with dark basaltic rocks. The geologic record indicates that Earth was a water-covered planet about four billion years ago, or earlier, with scattered sialic protocontinents [2]. Almost a billion years before the onset of plate tectonics, it was the Late Heavy Bombardment of young Earth (4.1 to $3.8 \mathrm{Ga})$ that is most likely the driving force for the origins of oceans, continents, and life [3].

Life arose through a long succession of chemical steps under the extreme conditions that existed on Earth four billion years ago. A new symbiotic model, describing the origin of life occurring in hydrothermal crater lakes, has been recently proposed $[3,4]$. In this scenario, life originated through five hierarchical stages of increasing molecular complexity: cosmic, geologic, chemical, information, and biological (Fig. 1). Each stage gave birth to a new sequence of biomolecules, with new characteristics, and increasing complexity that led to the emergence of the first cells. Each stage merged smoothly and continuously with the following stage. This multilayered hierarchy provided a kind of quality control at each assembly stage.

$<$ Figure 1 about here $>$ 
In the cosmic stage ( $\geq 4.6 \mathrm{Ga})$, life's first building blocks had their origin in the tiny icy grains of interstellar space, the result of supernova explosion of a nearby star. Some of these interstellar dusts formed into chondritic bolides that would bring the newly synthesized biomolecules to the Earth. During the intense meteoritic impacts of young Earth, carbonaceous chondrites delivered both the building blocks of life and water to the planetary surface creating innumerable crater basins $[5,6,7]$. Surface bombardment produces a unique thermal and chemical environment that was very likely the source of abiotic synthesis. The meteorite collisions that created hydrothermal crater lakes in the Eoarchean crust filled with cosmic water, organic molecules, and various hydrothermal fluids, gases, and energy; these inadvertently became the perfect crucibles for prebiotic chemistry [5].

Multiple lines of geological evidence point to a rapidly cooled Earth, blanketed with a liquid water ocean, dotted with felsic protocontinental islands, around four billion years ago $[8,9]$. This geologic stage begs the question 'Where did life begin on Eoarchean Earth?' There are currently two competing hypotheses for the site at which the first RNA/protein world emerged: hydrothermal vents in the deep ocean or hydrothermal crater lakes on land. Submarine hydrothermal vents are the currently reigning theory for the habitat of the origin and early evolution of life $[10,11]$. This theory suffers from the 'dilution problem' of the precise organic compounds [4]. The cosmic ingredients would be dispersed and diluted, rather than necessarily concentrated, in the vastness of the global Eoarchean ocean, preventing them from assembling into the complex molecules of life. A sufficient and necessary concentration of reactants is difficult to imagine in the open oceans. A protective barrier for the cradle of life is needed for prebiotic synthesis. Moreover, the low water activity in the hydrothermal crater vents allowed for an evaporative surface for polymer condensation; this mechanism rules out the open ocean as a 
viable environment. One crucial precondition for the origin of life is that comparatively simple biomolecules must have the opportunity to develop into more complex molecules through the segregation and concentration of chemical compounds, sequestered within the high crater rims. Because most chemical reactions proceed faster at high temperatures, hydrothermal crater lakes would be the ideal crucible for prebiotic synthesis. In the open ocean cosmic and terrestrial chemicals could never have mixed, concentrated, selected, or organized into more complex molecules. Moreover, submarine hydrothermal vent environments lack wet and dry cycles, which are well known to promote polymerization of nucleic acids and proteins [7].

Recent work suggests that the chemistry of modern cells still mirrors the original environment in which life first evolved [12]. The chemical nature of terrestrial hydrothermal vents resembles the cell's composition of its cytoplasm more closely the open ocean environment. Thus, protocells must have evolved in habitats with a high $\mathrm{K}^{+} / \mathrm{Na}^{+}$ratio and relatively high concentrations of $\mathrm{Zn}, \mathrm{Mn}$, and phosphorous compounds as in terrestrial environments. Geochemical reconstructions show that the ionic composition conducive to the origin of the cells could not have existed in marine settings but is rather compatible with emissions from vapor-dominated zones of inland geothermal systems such as hydrothermal ponds and crater lakes. From these observations, Mulkidjanian et al. [12] concluded it is unlikely that life evolved in the sea; instead, they champion the terrestrial, hydrothermal origin.

There is growing evidence against the theory of submarine hydrothermal vent origins in recent years, which suggest that the lipid membranes and nucleotides could not have been formed in that environment during prebiotic synthesis [13]. Amphiphilic compounds, such as fatty acids, readily form membranous vesicles when dispersed in terrestrial aqueous phases. Recent experiments suggest that these vesicles, precursors of protocells, are stable in 
hydrothermal hot spring water but are unable to assemble in seawater. In terrestrial setting, these vesicles can encapsulate nucleic acids such as RNA and DNA. These experiments clearly suggest that biogenesis started in terrestrial hydrothermal vent environments, not in the submarine hydrothermal vent setting. Similarly, nucleobases delivered by meteorites and interstellar dust particles to early Earth could only be polymerized into RNA in terrestrial environments such as warm little ponds or craters because of wet and dry cycles, a process that could not occur in deep ocean [14].

There are also major tectonic problems with the submarine vent hypothesis. The hypothesis of the submarine hydrothermal vents as a likely incubator is difficult to explain in a one-plate Eoarchean Earth. How did submarine hydrothermal vents, such as the black smokers or the Lost City, originate without plate tectonics? Today they occur along or near the axis of the spreading ridge. But since the plate tectonics did not start before $3 \mathrm{Ga}$ [15], there was no spreading ridge in the oceans for hydrothermal vent formation; we have to seek for alternative hydrothermal systems on land. Heavy Bombardment was the prime force in the early history of our planet before the onset of plate tectonics, creating thousands of craters on the crust of the young Earth.

Hydrothermal crater lakes are the most compelling environment for the cradle of life on early Earth during the Late Heavy Bombardment period [3, 4, 16-20]. This model provides a unique union of Heaven and Earth: from outer space the pristine building blocks of life were delivered to the Eoarchean crust through incoming asteroids, creating myriads of hydrothermal crater lakes, that became the crucibles in the origin of life. Thus, life is made of stardust, concentrated by Earth. The hydrothermal crater basins offered numerous possible niches for prebiotic chemistry: (1) continental crater lakes expose a variety of rock types including sialic, 
mafic, and ultramafic which provide a mineral-rich fluid to the hydrothermal soup, along with variable temperatures, magmas, and $\mathrm{pH}$ conditions; (2) terrestrial crater basins with low ionic compositions are compatible with that of modern cells thus favoring the likely site for life origin; (3) the prolonged convective circulation of heated water, as well as the temperature gradient allowed for the mixing and concentration of biomolecules; (4) sequestered high crater rims provided a physical compartmentalization for the biomolecules, a sequestered place where cosmic ingredients could concentrate and be thoroughly mixed with terrestrial hydrothermal chemicals, in time forming a thick, enriched solution of prebiotic soup; (5) alternate wet and dry cycles on the lake surface facilitated a hydration/dehydration phase which concentrated solutes through evaporation; (6) mineral substrates such as montmorillonite clay and pyrite in the crater floor aided in polymerization of nucleic acids and proteins; and finally; (7) biopolymers, such as polynucleotides and proteins concentrated in the nanopores of the mineral substrate from which lipid cells would encapsulate them into protocells (Fig. 2). The emergence of life was greatly facilitated by these seven prebiotic mechanisms that concentrated the chemical activities of the biomolecules in sequestered basins. The hydrothermal crater-basin itself served as the primordial cradle, providing the ideal conditions for multiple prebiotic chemical reactions and diversification, leading to the rise of living systems.

$<$ Figure 2 about here $>$

The greatest advantage of the cratering hypothesis is that it invokes the most probable scenario for the young Earth. Because of Late Heavy Bombardment, impact cratering was the most prevalent landform on the Archean crust. The surface of the oldest Greenstone belts, as the Vaalbara supercontinent, was probably pocked with thousands of craters, like the surface of the Moon and Mercury. Unlike our planetary neighbors however, the crater basins of Eoarchean 
Earth were filled with water and biomolecules and developed a complex network of hydrothermal systems (Fig. 3) [20]. Erosion, volcanism, and plate tectonics have erased almost all evidence of Archean impact history on early Earth.

$<$ Figure 3 about here $>$

In the chemical stage, geochemistry in conjunction with cosmic delivery of biomolecules that were present on early Earth must have defined not only the types of molecules that were present on hydrothermal crater lake environment, but also the molecular composition of early chemical systems and by extension that of protocells and contemporary cells. Furthermore, the hydrothermal vent environmental conditions must have defined the potential reactivity of the compounds. In the vent environment, cosmic and terrestrial chemicals were mixed, concentrated, and linked together by convective currents in these sequestered crater lakes, powered by hydrothermal, solar, tidal, and chemical energies; here, life began to brew (Fig. 2).

Oxidation/reduction (redox) reactions are key to supporting chemosynthesis within the vent environment. The hydrothermal lake water is abundant with potential electron acceptors (anions of various oxides). In contrast, abundant ferrous oxides and manganese oxides are reduced. High-temperature fluid/rock interaction forms reduced gases $\left(\mathrm{H}_{2} \mathrm{~S}, \mathrm{H}_{2}\right.$, and $\left.\mathrm{CH}_{4}\right)$ which dissolved into hydrothermal fluids. There was an abundance of chemical energy, such as ATP and phosphorous in the vent environment [29]. The cosmic biomolecules in the hydrothermal vents, entered into a complex series of 'far from equilibrium' chemical reactions. Abiotic chemical reactions in the hydrothermal vent produced a diverse array of molecules, from which only a few would be selected for the life building process. This selection may have occurred at different hierarchical levels, from small molecules to biopolymers to different protocells. Both the chemicals and the energy found in these hydrothermal crater lakes fueled most of the chemical 
reactions necessary for prebiotic synthesis and the resulting emergence of life. Monomers such as nucleotides and amino acids were selected from random assemblies of molecular pools and then polymerized on the pores and pockets of the mineral substrate to create RNAs and proteins to form the RNA/protein world [4, 21, 22, 23]. The establishment of a symbiotic relationship between proteins and nucleic acids was a landmark threshold in the evolution of life. These two biopolymers, with distinct structures and functions, became codependent. This interdependence of RNA for separate genetic functions, and protein for catalytic functions, provided the unique opportunity for enhanced feedback mechanisms, unavailable to single polymeric system such as RNA world. Most likely, pores and crevices of the mineral substrate of the crater floor acted as receptacles for concentrations of simple RNA and protein molecules [4, 24]. These macromolecules - specifically RNAs and proteins - are uniquely suited for the genetic, structural, and catalytic functions required for life. They are the information- and functioncarrying molecules of life on Earth. The prebiotic proteins in the RNA/protein world were mostly enzymes, adapted to the high temperature vent environment, capable of catalyzing the chemical reactions in the prebiotic soup.

In the chemical stage, the ability of the lipid membranes to encapsulate various monomers and biopolymers was crucial in terms of efficiency, stability, and molecular symbiosis. Encapsulation further insured the concentration and protection of life-encouraging ingredients from the vent environment, enhancing further biosynthesis [4]. Molecular symbiosis among membranes, RNAs, amino acids, and proteins was the driving force for the origin of complex cellular components. Lipid membranes randomly encapsulated RNA and protein molecules from the mineral substrates of the crater floor to initiate a molecular symbiosis inside 
the protocells that led to the hierarchical emergence of several cell components and their functionalities: first plasma membranes, then transfer RNAs, and finally messenger RNAs.

The chemical stage led gradually to the information stage. The putative transitions must have been involved to convert a dynamic, molecular chemical environment into a coherent, interconnected network of chemical processes, leading ultimately to the information age. The emergence of information carrier molecules such as the various RNAs became proactive and began to interact with peptides and proteins. In this information stage, replication and information storage were needed for a self-sustaining system to survive. These information processing functions were the culmination of the RNA/protein world. The molecular attraction between different species of RNA and corresponding amino acids led to the beginnings of the information stage. In this stage, the emergence of information system led to the synthesis of proteins by the translation of encoded RNA. This radical transformation in the prebiotic synthesis took place incrementally step by step creating the genetic code. Three classes of RNA molecules, mRNA, tRNA, and rRNA were important players in the expression of genetic information: mRNA was the initial storage molecule of genetic information, tRNA was the carrier of specific amino acids, and rRNA was the essential constituent of the protein producing ribosomes. The interactions between diverse RNA molecules and the myriad of amino acids and enzymes led to the gradual evolution of translation and the genetic code. As these two molecules began to develop in concert, the mRNA specified, in triplet code, the amino acid sequence of proteins. RNA molecules and amino acids began to communicate in different languages via bilingual enzymes that allowed biomolecules to cooperate with each other, leading to information systems and translation. 
The question of the origin of life turns out to be the question of the origin of biological information. The information systems probably arose in the RNA/protein world during the encapsulation of these biopolymers when rudimentary translation system and genetic code began to emerge [4]. The RNA/protein partnership performed two major functions during the origin of translation: storing information and catalyzing chemical reaction. They also began recognizing and utilizing different types of information system as the information system likewise evolved with them. The key processes of the Central Dogma (information flow) of molecular biology emerged simultaneously and naturally from the coevolution of RNA and enzymes [21]. With the emergence of ribosomes, mRNAs and newly synthesized proteins acquired information processing capabilities in digital and analog formats respectively. As information was stored in symbolic languages of nucleotides and amino acids, biosynthesis became less random and more organized and directional. With the advent of DNA, genetic information began to flow from DNA, to mRNA, to protein by a two-step process: transcription and translation.

In the biological stage, DNA replication was central to the binary fission and reproduction of the first cell. Reproduction was the culmination of the long journey of biogenesis that began with the cosmic gift of biomolecules. With the onset of binary fission, the population of primitive cells grew fast in the hydrothermal vent environment, evolved into cyanobacteria that invaded the oceans. Once life emerged, it evolved, thrived, and diversified across the Earth.

The hierarchical stages of the origin of life have been discussed in detail elsewhere and will not be repeated here except for background information $[3,4,14,15]$. In this paper we discuss primarily the background of prebiotic synthesis to address the origin of the information system, which is arguably the central and most difficult problem in the study of the origin of life. The purpose of this article is to offer new views on the origin of an information system in the 
prebiotic world during the emergence of translation and the genetic code. Although it is difficult to define what makes life so distinctive and remarkable, there is a general agreement that its informational aspect is a key property, perhaps the key property [25]. Since the discovery of DNA, biologists assign an ever-increasing importance to the role information plays in living systems. The Central Dogma summarizes the flow of information: DNA codes for mRNA, which in turn codes for proteins.

Recently, Walker and Davies [26] claimed that the genetic software provides a singular definition about what life is. They postulate that life emerged in that instant when information gained control over the biomolecules. According to them, life is fundamentally a phenomenon of information. Nucleic acids specialize in the use of chemical complementarity to encode information. The information-directed protein synthesis is a unique signature of life. According to Walker and Davies [26], biological information separates life from nonlife.

We agree with their view of an algorithmic origin of life. We suggest that life is more sophisticated than a digital computer where the software/hardware dichotomy is blurred and integrated. We find the computer analogy too simplistic. Both the informational and functional biopolymers in the translational machinery can be viewed as highly mobile molecular nanobots, which are fully equipped with both the information and the material needed to accomplish their tasks. These nanobots 'know' how to put themselves together by self-assembly or by cooperation with other molecules. It is our proposition that these complex molecular characteristics of life actually appeared before first life. These molecular nanobots are complex, self-replicating and self-managing information systems in themselves, analogous to the 'Universal Constructor' (UC) conceived by von Neumann [28]. UC is a self-replicating machine in a Cellular Automata (CA) environment. We expand on this idea later in the paper. 
The hydrothermal vents were incredibly crowded places, filled with exquisite nanobots moving around, bumping against each other, communicating, and doing specific jobs during the prebiotic synthesis. In the crowded RNA/protein world, a single mRNA nanobot serves as datastorage systems which receives another, charged tRNA nanobot; the two together then transmits digital instructions to the ribosome (a more complex nanobot) that in turn manufactures protein molecules in an analog format. Similarly, enzymic nanobots make, break, and rearrange the bonds holding other molecules together. The living cells have developed the extraordinary capabilities to store and process information, which are more efficient by many orders of magnitude than electronic digital computations, with respect to both information density and energy consumption.

To begin to understand how nature invented highly complex and specialized information systems from the vast array of disparate possibilities, we began this quest by running computer simulations of the major biosynthetic steps that might help explain the emergence of the system. In this paper, we use the Model-View-Controller (MVC) architecture [27] to reconstruct the molecular translation machinery; building our model from the components known to have existed in the hydrothermal vents, such as amino acids, nucleotides, and various enzymes. Nature must have developed a blueprint that described the order of these components in the synthesis of protein. That blueprint was stored in a nucleotide sequence in the mRNA. In information systems, the MVC architectural pattern has been used for consolidating information together, processing it into a model, isolating it from its manipulation (controller), and then presenting the component (the view) that determines the output form of the product (the artifact). Simply speaking, a 'view' relates to the logic (code) that produces the 'output.' The 'model' directly manages the information, its associated logic, and its rules of application. The third component, 
the 'controller,' accepts input (the signal) and acts as a monitoring coordinator that mediates between the tasks of the view and the model. The major premise of the pattern is modularity and distribution of processing. MVC separates the three different aspects of information processing: the data (the model), the visual representation of the data (the view), and the interface between the view and the model (the controller). Here we use the MVC paradigm to trace the origin and evolution of the translation and the genetic code by computer simulation, mimicking possible biochemical pathways.

\section{The Emergence of Macromolecules in the Hydrothermal Crater lakes}

Terrestrial hydrothermal impact crater lakes have been proposed as the most plausible cradle of life's origin on Earth [4,16-20]. Chemical energy such as ATP, amino acids, sugars, phosphorous, hydrogen, and other compounds were available in the hydrothermal vent environments [29]. The building-block molecules such as amino acids, nucleotides, fatty acids, and sugars, delivered from cosmic sources, began to accumulate in the crater basins. These natural caldrons were powered by the hydrothermal convection current, in which formed a complex solution of thick, concentrated prebiotic soup. The soup was recharged with a host of chemicals from the hydrothermal vents that aided in the catalysis. Reactive vent molecules such as $\mathrm{H}_{2} \mathrm{~S}, \mathrm{CH}_{4}, \mathrm{NH}_{3}$, and $\mathrm{H}_{2} \mathrm{~S}$, as well as metabolic energy such as ATP mediated the energy flow in the chemical evolution.

Various prebiotic processes produced a bewildering diversity of molecules from the cosmic and vent ingredients, out of which limited molecules were selected to serve as essential starting materials for life, such as lipids, amino acids, nucleotides, ribose sugars, and phosphates, 
and a variety of protein enzymes. For such a mixture to move in the direction of life, selforganization would be necessary. That process would increase the concentration of some components of the mixture either at the expense of others or by new synthesis from raw materials available in the vent environment. A fundamental property of life is the high degree of molecular selectivity and organization. The emergence of life occurred within the prebiotic chemical process, involving the handiwork of natural selection in a hierarchical fashion. Natural selection is the driving force behind the appearance of macromolecules in the vent environment, leading to biochemical pathways of biosynthesis.

Three critical macromolecules—cell membranes, nucleic acids, and noncoded protein catalysts - were synthesized in the chemical stage. This is likely a point in time at which biology did not yet exist, but instead pure chemistry defined the protocellular reaction network. These three macromolecules were radically different, their chemistries seemingly incompatible, each arising from a different precursor; yet they would come together in cooperation and eventually give birth to the genetic code. The order of emergence of these complex biomolecules, long before the first cells, is one of the most controversial topics in the origins of life story. Most likely, membranes arrived first, in a freeze-dried state, delivered to young Earth during the Heavy Bombardment. The simultaneous origins of nucleic acids and noncoded proteins followed some time later [4]. During the emergence of these biopolymers, information entered into the system of biomolecules. Here we reconstruct the likely scenarios for the order of emergence of these biomolecules in the hydrothermal vent environment.

\subsection{Cell Membranes}


Lipid cell membranes were first to form from the cosmic ingredients through a system of self-assembly [30]. In the prebiotic hydrothermal crater basins, a secure boundary is needed to separate evolving living system from nonlife. The cell membrane maintains this division of the world into pockets of life, and the physical environment in which they must sustain themselves. Deamer et al. [7] found that organic compounds from the Murchison meteorite would form membranous vesicles when they interacted with water. The hollow spheres extracted from the Murchison meteorites mimic the cell membranes. These fatty molecules don't dissolve in water, but spontaneously form tiny vesicles which become much more stable in terrestrial water by forming bilayers. They have enough mechanical strength, to have both contained other cell components such as nucleic acids and proteins and to have given these components some protection from the outside. Lipids can undergo spontaneous self-organization into larger structures such as micelles and bilayers. The formation of the membranes is crucial to the development of cellular life. This process of membrane formation is driven by the cycles of hydration and dehydration within the fluctuating hydrothermal crater basins [31].

\subsection{The Selection of Homochirality}

Living cells are composed of pure left-handed amino acids and right-handed sugars, a property called chirality. The process by which life became homochiral is a deep clue to the origin of life which occurred in the chemical stage. Some critical biomolecules in the prebiotic soup such as amino acids and sugars produced chiral molecules in 50:50 mixtures. In an early stage of chemical evolution, left-handed (L) amino acids and right-handed (D) sugars were selected from heterogeneous populations for molecular complementarity [33]. Crystal faces of 
certain common minerals such as quartz, feldspar, diopside, and calcite might have been involved to separate chiral molecules from racemic mixtures [24,34].

\subsection{Polymerization}

The polymerization of monomers is the next key event in the chemical evolution that funneled large assortments of biomolecules into a few selected biopolymers. The catalytic nature of the mineral surface of the crater floor played an important role in the polymerization of RNA and protein molecules $[3,24]$. Experiments have shown that these condensation reactions occur readily when growing polymers stick to small mineral particles [24,34]. Several mineral catalysts for polymerization, such as montmorillonite clay and pyrites, abounded at the hydrothermal vent environments. These minerals provided the scaffolding for monomers such as amino acids and nucleotides. L-amino acids were linked on the pyrite surface to form proteins of different lengths. Similarly, nucleotides were linked on the montmorillonite surface with the D-sugarphosphate backbone forming RNAs. Molecular adhesion forces between the microscopic layers of minerals brought these monomers close together and linked them to form polymers [4]. The prebiotic monomers were activated by the loss of water molecules, or by the addition of phosphate for polymerization [27]. Condensation or loss of water (dehydration) between two monomer molecules facilitates linking them into long chains of polymers. The mineral surfaces and porous matrices at the floor of the crater basin could also encourage concentrations of lipid membranes that fostered encapsulation of biopolymers such as RNA and protein molecules forming primitive protocells. 


\subsection{RNA/Protein World}

Among several competing hypotheses for how life arose on Earth, the 'RNA world' model is widely accepted [31-40]. The RNA world has become the main paradigm in the current origin of life research in which RNA assumed informational and functional roles. RNA is similar to DNA, except that it primarily exists in single-stranded form. So, instead of forming an inert double helix structure like DNA, some RNA molecules can fold into a complicated threedimensional structure reminiscent of proteins - this allows them to be chemically active. Moreover, these RNA molecules such as ribozymes, can act as catalysts for chemical reactions between other RNA molecules. The discovery of catalytic RNAs, and the revelation that the ribosome is in fact a ribozyme, together added strong circumstantial evidence for the RNA world theory [41].

Despite the conceptual elegance of the RNA world, this hypothesis faces formidable difficulties, primarily, the immense challenge of RNA synthesis under plausible prebiotic conditions $[24,32,42]$. The RNA molecule is inherently fragile in the natural environment and constantly degrades into smaller fragments through hydrolysis, preventing faithful reproduction. During the polymerization of nucleotides, a steady input of proteins is essential for RNA replication. Conversely, amino acids could be polymerized easily on the mineral surface to form protein molecules [34]. Information in RNA is stored in digital format, but it is processed in an analog format through protein synthesis. If RNA plays both genetic and catalytic roles, there is no possibility of separating the information storage from the information processing; it contradicts the organization of information flow in living systems [43]. There is a growing consensus that RNAs and proteins appeared simultaneously during prebiotic synthesis, and their 
collaboration was crucial to the origin of life [4, 21-23]. The RNA world paradigm is shifting to an RNA/protein world paradigm. There is increasing evidence that RNA and protein molecules interacted very early on in the origin and evolution of life (rather than RNA and RNA worlds giving rise to proteins), even short proteins had significant catalytic capabilities. RNA and protein, two complementary molecules existing in the prebiotic environment, mingled and interacted to form a dynamic system; one cannot exist without the other. Given that life depends on a diversity of molecule types in a symbiotic effort, each interacting with the other in complicated ways, it is hard to imagine that it would have started with just a single type of molecule. Starting with the spontaneous emergence of small autocatalytic sets of various molecule types such as short RNAs and proteins, these reaction networks would then have evolved and become more complex, creating more efficient biomolecules ready to incorporate information system.

The duality of replication and metabolism is the intrinsic property of life and must have appeared simultaneously before the origin of the DNA [33]. RNAs provide instructions to build proteins with the help of various enzymes. The establishment of symbiotic relationships between proteins and RNAs was a fundamental threshold in the evolution of life. These two biopolymers with distinct structures and functions, became codependent. In the RNA/protein world, protein enzymes catalyze chemical reaction, stitch RNA strands together, transport molecules around the protocell, and control what enters and leaves through the membranes. Proteins aid the replication process of RNAs and ultimately enable structure and function — bringing genes to life.

Obviously, it is advantageous for the RNAs to replicate the helpful proteins along the way, and in so doing boost their own replication. The encapsulated hydrothermal environment of protocells was very likely densely crowded with small molecules, monomers, and functional and 
non-specific polymers. Crowding enhanced concentration through the replication of RNA, the selection of symbionts for endosymbiosis, and the evolutionary innovation through autocatalysis. RNA and proteins worked in tandem to expand their informational, structural, and functional repertoires. The fundamental property of life, replication and metabolism, is believed to have evolved in the RNA/protein world, where RNA stores genetic information and protein enzymes function as catalysts [33]. Proteins are like a box of Legos: By changing the sequence of amino acids, any shape of enzyme can be constructed. Using the trial and error of linking amino acids in vent environment, myriads of enzymes have evolved, each custom-made to catalyze a particular reaction. Protein enzymes are obviously superior to RNA as catalysts because of their greater repertoire of chemical moieties and structural flexibility. Conversely, proteins are vastly inferior to RNA for the storage of genetic information because of absence of mechanisms for template-directed replication. The direct evolution of inherited genetic information coupled to encoded functional proteins, as is observed in real-world molecular biology, is far more plausible than any scenario in which there was as initial RNA world of ribozymes sophisticated enough to operate a genetic code [21]. Both RNAs and protein enzymes worked cooperatively to jumpstart the life assembly, each complementing the other. The dual systems of RNA and proteins were linked by a genetic code to begin communicating when an information system emerged. The transition from the chemical to the information stage is the most important step in the origin of life, because life is chemistry plus information.

\section{The Age of Information}


Despite the astonishing diversity and complexity of living systems, they all share five common hallmarks: compartmentalization, growth and division, information processing, energy transduction, and adaptability. Information processing arose as an emergent property in the RNA/protein world, an outcome of the complexity of the chemical stage (Fig. 1). In the hydrothermal vent environment, a large number of biomolecules began to interact locally, without a central control, yet the system as a whole began to perform sophisticated global information processing that would give rise to translation and the genetic code of living systems. Such global information processing, arising only from a local interaction in the RNA/protein world without a central control, is generally referred to as emergent computation. It is still not fully understood how such emergent computation was achieved in the information age, or how this ability evolved over time in biological systems.

Various protocells appeared in the information stage. A protocell should contain three components: a compartment (cell membrane), an information system (various RNA species), and catalytic machinery (noncoding protein enzyme). Another type of catalyst, such as metal ions of $\mathrm{Fe}, \mathrm{Mn}, \mathrm{Zn}$, and $\mathrm{Cu}$ were also available in the vent environment. Gradients of metal ions drive metabolism, and metal often centers in the active sites of enzymes. These components of protocells should work in an interconnected, symbiotic fashion to achieve the prolonged activity necessary for the protocell evolution. The primitive bilayer of lipid membranes was somewhat permeable, harvesting geothermal and chemical energy such as ATP from the environment [30]. The uptake and transduction of energy is essential for the emergence and evolution of protocells.

The informational and functional molecules such as RNAs and proteins have a high degree of specific complexity. The age of information introduces molecular communication and complementarity - the lock-and-key relationship — between RNA and proteins. The base pairing 
of RNA and its replication played a crucial role in building the information system. Different species of RNA would evolve to specify different functions in the information system.

Life is information stored in a symbolic language. Information is the currency of life. In all living cells, DNA is the ultimate repository of genetic information. There is a built-in, algorithmic information system in all cells. A genetic message, unique to that individual, is recorded in the digital sequences of the DNA of every living organism. The message in the genetic system is segregated, linear, and digital. The genetic information is essentially a digital data, plus meaning [28]. The base sequences of mRNA provide the data, and the meaning is the translation of the data into a functional protein. The flow of information system probably arose in the RNA/protein world during the encapsulation of these biopolymers when the rudimentary translation system and genetic code began to emerge.

A system that could store, reproduce, and translate the information required for protein synthesis was a critical event for protocells on through to living cells. The Central Dogma of molecular biology posits that information flows from DNA to RNA to proteins; transfer of information from proteins back to nucleic acids does not occur frequently in biological systems [44]. Koonin [45] provides a novel explanation for the irreversibility of the translation process. He suggests that the nucleic acids are a digital information repository, whereas proteins process information in an analog format. Koonin argues that the unique unidirectional route of information transfer represents the shift from digital to analog encoding information. In other words, the flow of information occurred from one-dimensional (digital) information contained in nucleic acids to the three-dimensional, analog form of information embodied in proteins. This irreversible translation process is the explanation for the non-heritance of acquired characters in evolutionary theory at the molecular level. 
The genetic code is the set of rules by which information encoded within mRNA sequences is translated into proteins. The genetic code maps the 64 nucleotide triplets (codons) to 20 amino acids. It can be seen as a dictionary that translates the four-letter language of the nucleic bases - A, U, G, and C of mRNA into 20-letter language of the amino acids. Translation is transferring information from the language of mRNA to the language of proteins. During translation, mRNAs serve as a data-storage system, transmitting digital instruction to molecular machines, the ribosomes, that manufacture protein molecules. RNAs are essential in encoding information. Three kinds of RNA molecules play major roles in translation: The messenger RNAs (mRNAs) carry genetic information to the ribosomes where proteins are synthesized. The transfer RNAs (tRNAs) function as adaptors between amino acids and the codons in mRNA during translation. The tRNAs also carry the specific amino acids to the ribosome during protein synthesis; they are the handler by which the mRNA is pulled through the ribosome via-codon anticodon interactions in the course of translocation. The ribosomal RNAs (rRNAs) are structural and catalytic components of the ribosomes. Arguably, the ribosomes are the most intricate and sophisticated nanomachines in nature that translate nucleotide sequences of mRNAs into amino acid-sequences of proteins.

The RNA-based information system depends mostly on enzymic proteins for replication and translation of the nucleic acids. However, the specificity of the enzyme depends on their amino acid sequences, which are determined by the sequences of nucleotides in RNAs. In the beginning, amino acids were utilized by ribozymes as cofactors, developing complex interactions between different RNAs and amino acids that led to the origin of translation and genetic code. Several enzymes were essential for protein synthesis including ribozymes, peptidyl transferase, and aminoacyl-tRNA synthetase (aaRS). 


\section{Temporal Order of Emergence of the Translation Machinery Systems}

Polymers are a fundamental component of life. Biopolymers-specifically, RNAs and protein enzymes - form the genetic and catalytic species, which evolved and changed over time - during the information stage. The oligomerization of monomers such as amino acids and nucleotides likely preceded the emergence of evolving biopolymers such as proteins and RNAs. In our model, proteins and RNAs were interdependent even in ancestral forms; they may be the products of evolutionary processes that acted on protobiopolymers that are no longer extant in living cells $[3,4]$. The coevolution of symbiotic relationships between multiple polymeric systems could be facilitated by the encapsulation of protocells, allowing the retention of successful outcomes while remaining separate from competitors within the hydrothermal vent. Some of the protobiopolymers such as pre-tRNA, pre-mRNA and pre-aaRS drove further polymerization and achieved informational competency and catalytic ability for initiating rudimentary translation. The processes of early macromolecular chemical evolution were intrinsically linked to energy transduction, especially to mineral-driven condensation processes in the hydrothermal environment.

\subsection{Translation Machinery Complex}

We have now some idea about the primitive milieu in the hydrothermal crater vent environment in which the genetic code originated in the RNA/protein world. The prebiotic soup was a rich collection of biomolecules in a highly reactive environment, owing to the constant 
input of hydrothermal energy. Some of these biomolecules were selected and encapsulated in protocells. The origin of the translation system is hotly contested before the origin of DNA: it is the central and the most difficult problem in the study of the origin of life $[35,43,45,46]$. We are of the opinion that the transition from noncoded protein to coded protein in the RNA/protein world might have given rise to the translation system and the genetic code. This remarkable development could have occurred incrementally by natural selection as the demand for more and more efficient and specific protein enzymes became greater than the supply for protocellular functions. The solution that arose was the translation system - the recipe for making custommade proteins.

In the RNA/protein world, protein enzymes played significant roles in accelerating the chemical reactions, by lowering activation energy. Enzymes catalyze metabolic processes in living systems. In the prebiotic synthesis, ancient protein enzymes adapted to the hot hydrothermal vent environment, where they jump-started essential chemical reactions and ensured efficient catalytic rates. As a protein, an enzyme has a unique three-dimensional shape, and that shape determines which chemical reaction the enzyme catalyzes. Enzymes differ from most other catalysts by being more specific about what substrates they bind and then catalyze. Since the substrate must fit into the active site of the enzyme before catalysis can occur, only properly designed molecules can serve as substrates for a specific enzyme; in many cases an enzyme will react with only one naturally occurring molecule. The specificity of each enzyme results from its unique sequence of the 20 amino acids, thus giving it unlimited diversity. Each enzyme is specific to a substrate, each complements the other. Thus, there was a coevolution of enzyme and its substrate (such as RNA) during prebiotic synthesis that gave rise to more and more complex molecules. RNA and proteins worked in tandem to expand their informational, 
catalytic, structural, and functional repertoires. This is why wide range of enzymes were required from the beginning to catalyze array of chemical reactions. The role, availability, and refinement of enzymes for a wide range of protocellular functions must be a key characteristic.

Amino acids are the fundamental building blocks of proteins, which are the main catalysts that support life. How amino acids were produced under early prebiotic conditions is an essential question to address the origin of noncoded proteins. Ten proteinogenic amino acids were abiotically synthesized under hydrothermal conditions. However, through the linking of natural amino acids, the prebiotic processes gave rise to a limited variety of random, noncoded enzymes; most were useless, without a suitable substrate, but a few were specifically selected for their matching substrate. The need for both specific and a wide range of enzymes became essential in the RNA/protein world. Darwinian selection provided the driving force for the evolution of specific coded protein over noncoded protein, so that protein synthesis became essential to the protocell function. These coded proteins were custom-made by translation machines consisting of a repertoire of RNA and protein molecules, and were highly specified for protocellular functions. The evolution of coded protein was a long evolutionary process driven by the incremental advances of the translation machinery which facilitated the transition from random, simple, noncoded proteins produced through an abiotic process, to the eventual production of specific, complex, coded proteins by RNA-directed protein synthesis. Here we propose that the transition from noncoded to coded protein synthesis may well have led to the emergence of the information stage where the translation systems and the genetic code evolved concurrently with the also evolving translation machines. The proposed transition mechanism from noncoded to coded proteins, aided by the translation machines, provided a continuity of functions so that each subsequent step was an improvement. 
The synthesis of coded protein in the chemical stage was the missing link for the development of the genetic code and provided a viable mechanism for its origin. A protein is a long, linear chain of amino acids containing more than 20-30 residues. Shorter amino acid chains are called polypeptides. The genetic code initially began from ten amino acids which were present in the vent environment. The abundance of amino acids in the prebiotic environment, and the known catalytic activities of short peptides, make it logical to look for the early involvement of amino acids during the evolution of the coded protein. The first set of amino acids later served as precursors for the formation of the second set of amino acids. During this evolution of the coded proteins, various components of the translation machine such as tRNA, aaRS, mRNA, and ribosomes would first appear sequentially and work in unison. An important aspect of the specificity between an amino acid and its corresponding tRNA and aaRS is that once the specificity is established, a mechanism for 'memorizing', or encoding the variations in the sequence of mRNA becomes possible. We will discuss in a later section how ten more, completely new amino acids were synthesized from their precursors during the coevolution of the translation, genetic code, and the coded proteins, along the prebiotic pathways. Thus, amino acids and their final products, the coded proteins, are the two end points in the long chain of translation and the genetic code.

The evolution of complex information systems must consist of plausible, elementary steps, each conferring a distinct advantage on the evolving ensemble of genetic elements. Here we map the emergence of potential informational and catalytic oligomers, derived from the assembly of building blocks in the crater vent environment, and reconstruct the probable steps leading to the translation machinery and the genetic code. The most important steps include: base pair complementarity, the origin of ribozyme, the origin of tRNA, the origin of aminoacyl-tRNA 
synthetase, the origin of mRNA, the origin of ribosome, the synthesis of protein, and the origin of the genetic code and translation. At the end, we apply the Model-View-Controller architecture, simulating the possible biochemical pathways in the design and development of the information systems of life.

The translation apparatus is an extremely complicated hierarchy of complex macromolecules that are related to one another in complex ways. Yet the whole functions with astounding precision. The ubiquity, conservation, and importance of a translation system provides a window to understand how RNAs and peptides became codependent. Protein synthesis is the most complicated and fundamental biochemical processes by which individual cells build their specific proteins. It is well-known that modern protein synthesis proceeds with the participation of 20 amino acids, ribozymes, tRNA, various enzymes including aminoacyl tRNA synthetase (aaRS), mRNA, ribosomal RNA, ribosomal proteins, ribosome, a considerable number of proteinous factors, ATP, GTP, etc. More than 120 species of RNAs and proteins are involved in the process of protein synthesis [47]. The origin and early evolution of the genetic code cannot be discussed without the evolution of these components - especially amino acids, tRNA, aaRS, mRNA, and ribosomes - as these components must have sequentially coevolved with the genetic code.

The translation system is ancient and highly conserved and must have started with protobiopolymers [46]. It is most likely that the translation system employed by the cell today has undergone a most extensive and involved evolution; but we don't know process because the transitional stages have been lost in time. Modern translation requires at least five kinds of macromolecules and amino acids: the set of tRNAs, the set of activating enzymes, the set of amino acids, mRNAs, and ribosomes. Most likely the evolutionary beginnings of translation 
could not have involved the interaction of all these components. Thus, the first assumption we need to make is that the beginning of translation involved a plausibly, small number of ancestral macromolecules with similar functional capabilities [48]. In our view the ancestral forms of tRNAs, mRNAs, and aaRS along with amino acids were used in the initial stage of translation. Most likely, the ribosome was the last molecular component to appear in the translation machine assembly.

Here we start the translation system with two distinct evolutionary precursor macromolecules: pre-tRNA and pre-aaRS that would design and tailored small pre-mRNA molecules for storage information and initiate translation. Eventually these macromolecules would evolve into tRNA, mRNA, and aaRS. Finally, as the fidelity of translation was refined, ribosomes appeared on the scene, making the protein synthesis more efficient.

We identify seven major stages for the origin and evolution of the translation machinery complex and the genetic code leading to the protein synthesis. A crucial initial step was the chemical bonding of particular amino acids to small RNA molecules with specific base sequences. Initially, ribozymes utilized amino acids as cofactors that led to the emergence of different species of RNA molecules [51]. A stereochemical relation between some amino acids and cognate anticodons/codons is likely to have been an important step in the earliest assignments of amino acids. These possible biochemical pathways are: (1) the selection of amino acids; (2) the origin of RNA; (3) the origin of ribozyme; (4) the origin of transfer RNA; (5) the origin of messenger RNA; (6) the origin of ribosome; and finally, (7) protein synthesis. During the emergence of these biochemical pathways, the genetic code and translation system evolved and refined concurrently in the hydrothermal vent environment. 


\subsection{Selection of Amino Acids}

Amino acids contain amine (-NH 2$)$ and carboxyl (-COOH) functional groups, along with a side chain (R group) specific to each amino acid. All amino acids have the same general structure: a central carbon, bounded to $\mathrm{NH}_{2}$ - an amino functional group, a carboxyl functional group, a hydrogen atom, and a side chain or R-group. The 20 amino acids, used in protein synthesis, are different because their R-groups are different. The R groups of amino acids are highly diverse. The 20 amino acids can be sorted into 3 groups according to whether the R-group is nonpolar, polar, or electrically charged. The nonpolar R-groups are hydrophobic, and do not react with water. In contrast, amino acids with polar or charged R-groups react easily with water and are termed hydrophilic. These features make amino acids the most diverse and versatile of building blocks in nature and their preferential selection of tRNA would trigger the information age step-by-step. The hydrophobic or hydrophilic nature of amino acids would play an important role in the evolution of the genetic code.

Carbonaceous chondrites carry a large number of amino acids and were probably the major source of naturally-occurring amino acids in the hydrothermal crater lakes. A large pool of amino acids was available in the crater vent environment. Pizzarello and Cronin [49] identified 70 amino acids in Murchison meteorite. It is likely that similar numbers of amino acids were present in the prebiotic environment. Similarly, Miller's experiments have produced more than 40 different amino acids [50]. Out of 70 amino acids likely present in the crater-lake environment, only ten L-amino acids, which were most easily formed in the primordial soup, were selected and recruited through molecular recognition, by tRNA and its corresponding aminoacyl-tRNA synthetase; these ten amino acids were precursors for the formation of other ten 
amino acids along prebiotic pathways. The choice of ten primordial amino acids from prebiotic soup for synthesis of noncoded protein enzymes may have been the first product of molecular selection in the information age.

\subsection{The Origin of RNA}

The basic constituents of RNA molecules such as D-ribose, phosphate, and the four bases - adenine (A), guanine $(\mathrm{G})$, cytosine $(\mathrm{C})$, and uracil $(\mathrm{U})$, along with unused nucleotides were delivered to the hydrothermal crater lakes by meteorites. Polymerization of RNA molecules occurred by mineral catalysis. Nucleotide monomers were linked on the montmorillonite clay substrates of the crater floor in an ATP-rich environment $[4,24,30]$. The accumulation of phosphates in the vent environment was an important requirement for making the sugarphosphate backbone of RNA. Nucleotides are joined together by a phosphodiester linkage between $5^{\prime}$ and $3^{\prime}$ carbon atoms to form nucleic acids. The polymerization reaction involves the formation of a bond between the phosphate group of one nucleotide and hydroxyl group of sugar component of another molecule. The result of this condensation reaction is called a phosphodiester bond. In the prebiotic environment, nucleotides underwent spontaneous polymerization with the loss of water. The resulting product was a mixture of polynucleotides that were random in length and sequence.

Six hypothetical stages for the formation of the RNA molecules in the crater vent environment are shown in Fig. 6. It seems unlikely that the prebiotic soup in the vent environment produced only the four bases found in RNA - A, U, G, and C - which formed the polynucleotide chain. Certainly, there were other nucleotides (including hypothetical $\mathrm{F}$ and $\mathrm{N}$ 
bases in Fig. 4), which were incapable of Watson-Crick base pairing. Initially, all these mononucleotides were polymerized randomly into short oligonucleotides of different lengths by peptide bonding [42]. The process was mediated by natural selection and RNA replication. Natural selection led to the elimination of useless random oligonucleotide sequences during base pairing. From these chaotic assemblages of oligonucleotides, only four bases such as A, U, C, and $\mathrm{G}$ were selected by exploiting the properties of the Watson-Crick base pairing, whereas hypothetical $\mathrm{F}$ and $\mathrm{N}$ bases were eliminated. The four standard bases are better than 2 or 6 based on estimates of arbitrary catalysis and the actual pairing energy of standard bases [51]. The four nucleotides were strung together to produce short pieces of oligonucleotide and RNA molecules, which could replicate with the aid of peptide enzyme. Replication selected prebiotic RNA molecular bases from overwhelmingly large assortment of mononucleotides. These RNA molecules were random and noncoded, a jumble assortment of nucleotide bases.

$<$ Figure 4 about here. $>$

Once some rudimentary template-dependent synthetic mechanism allowing base-pairing was in place, molecules rich in A, U, C, and G then were progressively selected and amplified. These bases joined to form primordial RNA strands of different lengths, which began to selfreplicate through a process of base pairing. Short sequences of nucleotides are normally better replicators than long sequences. Longer sequences suffer from an important evolutionary disadvantage; it takes longer to replicate a long sequence than a short once. If a pool of nucleotide sequences containing a range of length is left to code and replicate, short sequences will dominate, and long ones will become extinct [52]. The base pairing principle would give rise later to codon-anticodon hybridization, the origin of messenger RNA, transcription, and replication. 
RNA is generally single-stranded and an informational molecule. Self-replication of RNA molecules occurs through a process of base pairing and dissociation. When one RNA strand is made in the vent environment, a second strand would form automatically through base pairing in such a way that cytosine always pairs with guanine, while adenine always pairs with uracil. Consequently, pairing is always between purine and pyrimidine. Because the hydrothermal vents in the crater basins were hot, double-stranded RNA, formed by base-pairing, came apart through dissociation of the two chains. When the strands separate, the cycle repeats with another round of base pairing leading to two more double-stranded RNA molecules, one of which contains the original strand, containing its exact copy. By exploiting the properties of nucleotide base-pairing, coupled with the high temperatures of hydrothermal vent in the crater basin, short pieces of RNA replicated without the aid of any other molecules. Such complementary templating mechanisms lie at the heart of RNA replication, producing a large, more diverse population of RNA molecules (Fig. 4).

Because RNA contains a sequence of bases analogous to the letters in a word, it can function as an information containing molecule. Moreover, RNA being a single chain, is free to take any kind of shape; the structure it can achieve by morphing its shape is wide-ranging, similar to protein. From this basic architecture of a single-stranded RNA molecule, different species of RNAs such as ribozymes, transfer RNA (tRNA), messenger RNA (mRNA), and ribosomal RNA (rRNA) evolved inside protocells, with a supply of information, distinct in attribute and configuration in response to amino acids. There was a molecular choreography of different RNAs in the prebiotic world that led to the rudimentary translation. The advent and multifunction of different species of RNA molecules signal the transition from the age of chemistry to the age of information. 


\subsection{The Origin of Ribozyme}

The RNA molecule has a secondary structure. It can form a localized double-stranded RNA stem by base pairing, and a terminal loop to form a hairpin structure. In the stem, adenine forms a bond with uracil, and cytosine pairs with guanine to form double-stranded RNA. The resulting hairpin structure is a key building block of many RNA secondary structures such as ribozyme and transfer RNA (tRNA) (Fig. 5). As an important secondary structure of RNA, an RNA hairpin can direct RNA folding, determine interactions in a ribozyme, protect structural stability for messenger RNA (mRNA), provide recognition sites for RNA binding proteins, and serve as a substrate for enzymatic reaction [53]. Structurally, RNA hairpins can occur in different positions within different types of RNAs; they differ in the length of the stem, the size of the loop, the number and size of the bulges, and in the actual nucleotide sequence.

Ribozymes are RNA molecules that are capable of catalyzing specific biochemical reaction, similar to the action of protein enzymes. Ribozymes, assembled in the hydrothermal vent environment, could replicate themselves. There are different classes of ribozymes, but all appear to be associated with metal ions, such as potassium or magnesium. Different ribozymes catalyze different reactions, but almost all ribozymes are involved in catalyzing the cleavage of RNA chains or the formation of bonds between RNA strands.

Most likely, the chemical bonding of a particular amino acid to a small RNA hairpin structure led to the origin of ribozyme in the vent environment. We assume that different kinds of RNA, protein enzymes, nucleotides, oligonucleotides, and amino acids were available in the hydrothermal soup. The single-stranded nature of RNA molecule can be bent back on itself, in a 
hairpin loop, where the stems of the loops are maintained by base pairing to form threedimensional structure, just like a protein molecule to act as an enzyme. In some stem-loop configurations, two ends of the stem might remain free, containing the $3^{\prime}$ and $5^{\prime}$ ends. This $3^{\prime}$ end might function as an acceptor stem to form a covalent attachment to a specific amino acid (Fig. 5). This small hairpin RNA molecule with specific terminal base sequences acquired corresponding amino acid as a 'cofactor' to improve the catalytic range and efficiency to become initial ribozymes [51]. Many enzymes act with the help of one or more cofactors. Binding of amino acids to a ribozyme resulted in an enhancement of catalytic activity.

Any specific binding between two molecules involves information, as if two molecules 'recognize' each other. An amino acid can be linked to an oligonucleotide with three bases by an activating enzyme; the charged oligonucleotide is then bound on the surface of a ribozyme by base pairing and delivers the appropriate amino acid (Fig. 4). In this way, ribozymes are capable of producing short peptide chain. Overtime, the original peptide forming ribozymes will specialize as amino acid specific adaptors. In the RNA/protein world, different kinds of protein enzymes were available. Initially, one kind of an amino acid and one kind of hairpin would be catalyzed by an activating enzyme, perhaps precursor to the aminoacyl transfer tRNA synthetase (pre-aaRS). Each pre-aaRS is specific for an amino acid and for its corresponding ribozyme. Later, a second amino acid, attached to a different hairpin by a different ribozyme, would be added, and so on to create a chain of protein. It is our contention that the interacting union of a hairpin ribozyme with a specific amino acid is the cornerstone for the origin of information, transfer RNA, translation, genetic code, and protein synthesis.

$<$ Figure 5 about here $>$ 
A ribozyme has a well-defined tertiary structure that enables it to act like a protein enzyme in catalyzing biochemical and metabolic reactions. The relevance of ribozyme for the origin of tRNA is enormous. Ribozymes, assembled in the prebiotic vent environment, could not only replicate themselves but would catalyze the formation of specific proteins. The adaptor ribozymes are the precursors of tRNA molecules and play critical roles in the building of ribosomes. Ribosomal RNA functions as a peptidyl transferase in ribosomes to link the amino acids in protein synthesis, but the framework of transferase is provided by the ribosomal proteins.

\subsection{The Origin of Transfer RNA}

Any model for the development of protein synthesis must necessarily start with direct interactions between RNAs and amino acids. Chemical considerations suggested that direct interactions between the amino acids and the codons in mRNA were unlikely. The protein and mRNA languages seem unrelated. Amino acids do not read their codons. Thus, Crick [38] proposed that some kind of an adaptor molecule must mediate the specification of amino acids by codons in mRNAs during protein synthesis. The adaptor molecules were soon identified by other researchers as transfer RNAs (tRNAs), which serve as a reading device of mRNA through base pairing. The tRNA molecule binds to amino acids, associates with mRNA molecules, and interacts with ribosomes to decipher and translate the code of mRNA.

It is generally believed that the first RNA gene, the $U r$-Gen, was a precursor of modern tRNA [54]. tRNA is the ancestor of all RNAs. It is an ancient molecule that has evolved very 
little over time. The phylogeny of ribosomes suggests that tRNA is an ancient component of ribosomes that arose in the early prebiotic world [22].

A tRNA molecule is short, typically 76 to 90 nucleotides in length, that serves as the physical link, a cipher, between the messenger RNA (mRNA) and the amino acid sequences of proteins [35]. Although the tRNA molecule is short, both its primary structure and its overall geometry are undoubtedly more complex than those of any other RNA species [55]. The translation of a message carried in mRNA into the amino acid language of proteins requires an interpreter. The amino acids themselves cannot recognize the codons in mRNA. The tRNA matches appropriate amino acids to the appropriate codons. To convert three-letters words (codons) of nucleic acids to the one-letter, amino acids of proteins, tRNA molecules serves as the interpreters during translation. Each amino acid is joined to the correct tRNA by a special enzyme, aminoacyl-tRNA synthetase (aaRS).

tRNA participates in two clearly distinct steps in the translation process. The first step comprises the reactions leading to the charging of the tRNA molecule with an amino acid. The second step comprises the complex reactions in which tRNA transfers its amino acids into a growing protein chain, in response to a specific codon. Chemical reaction catalyzed by the tRNA is simple - the joining of amino acids through peptide linkages. It performs the remarkable task of choosing the appropriate amino acid to be added to the growing protein chain by reading successive mRNA codons. The actual step of translation from mRNA into protein language occurs when amino acids and tRNAs are matched and joined. The translators that do this job are the aminoacyl-tRNA synthetases (aaRS). These enzymes are the only bilingual elements in the cell: they can recognize both amino acid and the corresponding tRNAs. They are the key element of translation, the links between the worlds of proteins and nucleic acids. The activation of tRNA 
occurs when a synthetase uses energy from ATP hydrolysis to attach an amino acid to a specific tRNA. There are twenty such synthetases, one for each amino acid. Together they make up the complete dictionary for protein synthesis in a cryptic form that relies on tRNAs for decoding into the anticodon language. Each type of amino acid can be attached to only one type of tRNA, so each type of organism has many types of tRNA, and more than 20 amino acids. There might be a coevolutionary process in which the anticodons and the corresponding amino acids were progressively mediated by natural selection. As ribosomes appear, tRNAs transport amino acids to ribosomes, where the amino acids are assembled into proteins.

Because of its molecular complexity, the origin of tRNA is controversial. The modern tRNA structure with its complex configuration and multiple functions might have originated from a simpler form, such as pre-tRNA molecules to select specific abiotic amino acids in the vent environment (Fig. 6A-6D). The pre-tRNA molecules with hairpin structures (stem and loop) might have evolved in some evolutionary stages of protein synthesis, originating from a linear chain of RNA [56]. The tRNA has a secondary and tertiary structure. In solution, the secondary structure of tRNA resembles a cloverleaf with three hairpin loops (Fig. 6E, 6F). One of these hairpin loops contains a sequence of three nucleotides called the anticodon that forms base pairs with the mRNA codon. The other two loops of the cloverleaf form a D-arm and a T-arm. The unlooped stem contains the free $3^{\prime}$ and $5^{\prime}$ ends of the chain. The CCA sequence at the $3^{\prime}$ end of the acceptor stem forms a covalent attachment to the amino acid that corresponds to the anticodon sequence. The CCA sequence of the acceptor stem offered a binding site for the amino acid. The $5^{\prime}$ terminal contains a phosphate group. Both the anticodon and acceptor stem sequence correlate with the role of amino acids in folded proteins [57]. The secondary structure tRNA molecule may provide some clue to its ancestral molecular configuration. The cloverleaf- 
configuration of tRNA can be derived from a folded ribozyme with a single loop and an attachment site for the amino acid at the end of a stem (Fig. 6E).

The most plausible scenario of the origin of the tRNA molecule is based on RNAs that exhibited simple catalytic functions such as ribozymes. Perhaps the precursor of tRNA started as a simple ribozyme with a hairpin structure (Fig.6A, 6B). This ribozyme acquired amino acids at its $3^{\prime}$ end as a cofactor (Fig. 5): that is, an amino acid was attached to a ribozyme and made it a more efficient catalyst [58]. One way of attaching an amino acid to a particular point on the surface of the ribozyme is at the end of a single-stranded unlooped stem of the hairpin, which is charged and begins to bind amino acid that enhances the catalytic function of the ribozyme. With the stabilization of the catalytic reactions, these ribozymes began to participate in the first catalytic cycles. This configuration of a ribozyme linking an amino acid at the end may be the starting point for the origin of tRNA, where the unlooped stems contain the free $3^{\prime}$ and $5^{\prime}$ ends of the chain. This amino acid attachment to ribozymes by specific assignment enzyme first occurred to make cofactors more efficient catalysts [51].

Many studies have suggested that the modern cloverleaf structure of tRNA may have arisen from a single ancestral gene by duplication of half-sized hairpin-like RNAs by passing through some intermediate structures $[56,58-63]$. The linkage of an amino acid with a ribozyme at the end with a hairpin loop might be the starting point for the origin of tRNA, a quarter size of the modern tRNA molecule [51]. The relevance of ribozymes in the origin of tRNA is enormous. The equivalent effect of gene duplication might be accomplished by a simple ligation of two identical hairpins of folded ribozymes to create double hairpins, a D-hairpin and a T-hairpin with an anticodon at the stem bases [64]. RNA ligation is a powerful driving force for the emergence of tRNA, joining two hairpin loops of ribozyme (Fig. 6C). During the evolutionary transitions of 
the pre-tRNA molecule, the double hairpin structure with the D-hairpin and the T-hairpin formed in the ancient prebiotic world, with both the anticodon and the terminal CCA sequence adjacent to the D-hairpin (Fig. 6D) [62].

$<$ Figure 6 about here $>$

The function of tRNA molecules depends on their precise three-dimensional structure. The cloverleaf tRNA folds into a more compact L-shaped tertiary structure, but each has a distinct anticodon and attached amino acid (Fig. 6H). One arm of the L-shaped tRNA structure has a minihelix with a single-stranded CCA end used for attaching a single amino acid; the other arm forms an anticodon loop, with three unpaired bases that may bind with the complementary codon of mRNA. Each tRNA molecule can carry one of the 20 different amino acids at its CCA minihelix end. Each type of amino acid has its own type of tRNA, which binds it and carries it to the growing end of a protein chain during the decoding of mRNA. The CCA end of the minihelix interacts with the large ribosomal subunit to form a peptide bond, and the loop end interacts with the small ribosomal subunit for decoding mRNA triplets through codon-anticodon interactions [57].

We suggest that this half-sized hairpin structure of the pre-tRNA molecule acquired some functional capacity for translation before the emergence of tRNA (Fig. 6C, 6D). The pre-tRNA molecule is the evolutionary precursor of the tRNA molecule. The contemporary full-length tRNA molecules could have been formed by direct duplication, or by the ligation of half-sized, hairpin-like structures - the pre-tRNA molecule (Fig. 6E). The acceptor stem bases and the anticodon stem/loop bases in tRNA in tRNA $5^{\prime}$-half and $3^{\prime}$-half fit together with the doublehairpin folding; this suggests that the primordial double-hairpin RNA molecules could have evolved to the structure of modern tRNA by gene duplication, with subsequent mutations to form 
the familiar overleaf structure [56,61]. In other words, two pre-tRNA molecules somehow fused together to form a tRNA molecule.

The half-sized pre-tRNA molecule with two loops (D-hairpin and T-hairpin) on one side, and anticodon and acceptor stem region of CCA end on the other side, is structurally and functionally independent and is more ancient than the other-half of the tRNA molecule [61]. This short, self-structured strand of pre-tRNA molecule possesses a template domain, which is chargeable through interaction with specific amino acids, is probably the predecessor of tRNA (Fig. 6C). This pre-tRNA molecule binds with high specificity to the amino acid corresponding to its anticodon; this reaction is catalyzed by a specific pre-aminoacyl-tRNA synthetase (preaaRS). tRNA evolution is closely linked to aminoacylation. There is a separate tRNA for each amino acid that carries a triplet sequences of nucleotides for anticodon. Later, anticodon of pretRNA will guide the codon formation of the pre-mRNA.

It should be apparent that tRNA molecules must contain a great deal of specificity despite their small size. Not only do they (1) have the correct anticodon sequences, so as to respond to the right codons, but they must also (2) be recognized by the correct aaRS, to be activated by the correct amino acids, and (3) bind to the appropriate sites on the ribosomes to carry out their adaptor functions.

An important aspect of the specificity between amino acids and pre-tRNA is that once this specificity is established, a mechanism for 'memorizing' or encoding variations in the sequence of pre-tRNA molecules becomes possible [65]. These pre-selected biomolecules of amino acids emerged from the existing prebiotic soup of the crater vent environments. Among the many essential components of the translation process, assignment enzymes evolved to bind a specific amino acid to a pre-tRNA molecule, (Fig. 6). 


\subsection{The Origin of Aminoacyl-tRNA Synthetase}

Aminoacyl-tRNA synthetases (aaRSs) are a superfamily of enzymes responsible for creating the pool of correctly charged aminoacyl-tRNAs, that are necessary for the translation of the genetic information (mRNA) through the ribosome. aaRSs are very ancient enzymes that are present in all organisms, and are one of the pioneer molecules formed by the polymerization of amino acids. The catalytic nature of mineral surfaces must have played an important role for polymerization and the linking of amino acids into protein molecules. Mineral-induced peptide formations are well known on pyrite surfaces because peptide-forming reactions involve a relatively simple mechanism [34]. The polymerization of proteins from amino acids requires the dehydration and condensation mechanism precisely found in the fluctuating hydrothermal crater basins. In the hydrothermal crater vent environment, various kinds of protein enzymes appeared through the polymerization of amino acids, but few were selected. The aminoacyl tRNAsynthetase (aaRS) enzyme is one of the primordial enzymes that was synthesized abiotically on the pyrite substrate of the hydrothermal crater vent environment. Each enzyme catalyzes the activation of a specific amino acid and recognizes a specific tRNA for binding.

Aminoacyl-tRNA synthetases plays a crucial role in translation by ensuring that amino acids are bound to their cognate tRNAs. The linkage of an amino acid to tRNA is important for two reasons. First, the attachment of a given amino acid to a particular tRNA establishes the genetic code. When an amino acid is linked to an tRNA, it will be incorporated into a growing protein chain at a position dictated by the anticodon of tRNA. Second, the formation of a peptide bond between amino acids is not thermodynamically favorable. The amino acid must first be 
activated for protein synthesis to proceed. The activation intermediates in protein synthesis are amino acid esters.

The activation reaction is catalyzed by specific aaRS. The first step is the formation of an aminoacyl adenylate with an amino acid and an ATP. The next step is the transfer of the aminoacyl group to a particular tRNA molecule to form aminoacyl-tRNA, or a charged tRNA. The mechanism of aaRS formation is well-known [57]. It reveals insight into how and why tRNA molecule creates its own bilingual enzyme aaRS that can then connect it with the appropriate amino acid. It enhances the selection and sorting of appropriate amino acids from the prebiotic soup for protein synthesis. Each aaRS is highly specific for a given amino acid. It has a highly discriminating amino acid activation site. Both amino acids and ATP were available in the hydrothermal vent, facilitating a reaction with tRNA to form aminoacyl-tRNA synthetase. Moreover, the proofreading ability by aaRS increases the fidelity of protein synthesis.

How do aaRS choose their tRNA partners? aaRS recognizes the anticodon loops and stems of tRNA molecules. This enormously important step is the point at which translation takes place - at which the correlation between the amino acid and nucleic acid is made. In a sense, aaRSs are the only molecules in the prebiotic world that recognize the emerging genetic code. Their precise recognition of tRNAs is important for protein synthesis as well as the accurate selection of amino acids. They are the matchmakers between a specific amino acid and its cognate tRNA. They ensure that proper amino acids are used to build proteins.

aaRSs come in twenty flavors, each one specific to an amino acid and tRNA. These twenty enzymes are widely different, each optimized to function with its own particular amino acid and the set tRNA molecules appropriate to that amino acid. They can be divided into two classes, termed class I and class II. We speculate that the precursor of aaRS was pre-aaRS, a 
hypothetical primordial ancestor that gave rise to two classes of aaRS, both are multidomain proteins. Each aaRS uses different mechanisms of aminoacylation. In our model, the original aminoacylation enzymes were pre-aaRS, a simpler version of aaRS, which must have featured a strong linkage to the anticodon of a pre-tRNA molecule. This linkage must have featured a codon-like, trinucleotide binding site for the adaptor's anticodon, on the pre-aaRS. Thus, we propose that the primordial synthetase is a protein enzyme, including an anticodon, plus a domain capable of binding and activating an amino acid and transferring to the pre-tRNA. Carter and Wills [21] called this ancestral form of aaRS 'urzymes.' pre-aaRS or urzymes from Class I and Class II aaRS are roughly $10-30 \%$ the size of full-length enzymes. Pre-aaRS contain two domains for catalytic and anticodon-binding functions respectively.

Enzymes promote chemical reactions by bringing substrates together in an optimal orientation, thus creating an ideal chemical environment. The enzyme's active site binds to the substrate. The enzyme will always return to its original state at the completion of the reaction. The aaRS, also called tRNA-ligase, is an activating enzyme that attaches the appropriate amino acid onto its tRNA. It does so by catalyzing the esterification of a specific cognate amino acid or its precursor to each one of the compatible cognate tRNAs to form an aminoacyl-tRNA. The aaRS can recognize both amino acids and the corresponding tRNA from a large pool of similar biomolecules. It is the linchpin of translation, the communication link between the worlds of proteins and nucleic acids.

Each amino acid has its own aminoacyl-tRNA synthetase, which recognizes its cognate amino acid and all of the cognate tRNAs for that amino acid. There are twenty different aaRS, one for each type of amino acid. There are two unrelated classes of these enzymes with distinct active site topologies, each class encompasses 10 of the amino acids. There are twenty such 
enzymes, one for each amino acid. Each enzyme has two binding sites, one for a tRNA, the other for an amino acid; both are situated in the vicinity of a catalytic site in such a way that the amino acid is attached to the tRNA. The aaRS is bilingual and can translate the nucleotide language of RNA into the amino acid language of protein. It has imprinted in its structure one line of a genetic dictionary, with all synonyms included. The aaRS serves simultaneously in activation (energy) and in translation (information). aaRS catalyzes the tRNA charging. It accomplishes its energetic function by using ATP to join amino acids with an tRNA molecule in such a manner that the energy of the created bond supports the peptide bond formation [66].

As adaptors between codons and amino acids, tRNAs are directly responsible for deciphering the genetic code. It is therefore critical that each tRNA is charged with its correct amino acid. Thus, synthetases have a twofold challenge: they must recognize cognate tRNAs and they must recognize cognate amino acids. Without aaRS, the link between amino acid and tRNA molecule could not be established. The evolutionary emergence of aaRS is pivotal to the beginning of translation. As we will come to, the aminoacyl-tRNA is the direct substrate for the peptide bond formation by the ribosome.

\subsection{The Origin of Messenger RNA and Translation}

In living cells, the genes in DNA encode protein molecules, which carry out all the functions necessary for life. In the first step, a segment of a single DNA strand is transferred to a mRNA molecule by transcription, where mRNA can specify the amino acid sequence of the protein products of the gene. There are two haunting questions regarding the genesis of mRNA: (1) how mRNAs first appeared in the prebiotic environment, before the emergence of DNA, and 
(2) how they evolved in the sequence of nucleotides, with the function of specifying amino acids as the fundamental components for the origin of the genetic code. The primordial mRNA was lost long ago in the information stage of biogenesis, leaving no trace of its origin. While existing evidence suggests that the genetic code was influenced by physico-chemical interactions between individual amino acids and strings of nucleic acids $[67,68]$, researchers have yet to piece together the stepwise mechanisms by which it evolved over time. Here we suggest a new model for the origin of an mRNA specially tailored by tRNA.

In the prebiotic world, different species of RNA evolved through cooperation, each with a different function. Although random RNA strands grow during prebiotic synthesis by base pairing, in which some portion of the strand may show codon-like arrangement of nucleobases, they did not contain any genetic information (Fig. 4). Moreover, the strings of nucleotide may be interrupted haphazardly by stop and start signals. A fundamental property of protein synthesis is that the amino acids are not added in haphazard fashion. Their sequence is rigorously imposed by mRNA, which is itself formed by tRNA incrementally. Each mRNA must be specially made, specific to each protein.

Here we propose a new model for synthesis of custom-made mRNA by tRNA. The evolution of non-random coding mRNA served as the first medium for genetic information that coincided with the development of the genetic code and protein synthesis. As the tRNA molecules began to recognize and react with certain amino acids, they need a separate storage device for safe keeping the information of amino acid assignment. Because the selection of mRNA depends exclusively on codon-anticodon interaction, tRNA begins to make a specific strand of mRNA for storage of amino acid information (otherwise, it is difficult to see how else mRNA molecules could have become involved with coding the strings of amino acids in a 
specific manner). Here we suggest the origin of a new generation of ancestral mRNAs - premRNAs, were created by ancestral tRNAs or pre-tRNAs step-by-step. At this stage, the information begins to flow upstream from pre-tRNAs to pre-mRNAs. These newly synthesized pre-mRNAs have direct preferences for amino acids they tend to encode. Woese [55] suggested that amino acids in ancient systems associated with mRNA directly in the course of translation, following their intrinsic physicochemical properties. This association may reveal clues of complementary interactions between mRNAs and their cognate proteins.

We suggest that pre-tRNA molecules begin to select codons via base pairing with their anticodons; these short codon segments are linked to create longer strand of pre-mRNA step-bystep for storing genetic information. In the pre-tRNA molecule, the site of attachment of the appropriate amino acid is proximate to the anticodon, making communication between two active sites easier (Fig. 7A, 7B). The physical proximity of the anticodon and the acceptor stem in ancestral pre-tRNA molecules is relevant to a long-sought goal - deriving amino acid/codon pairing rules from an ancestral nucleotide-based receptor-ligand recognition system [48]. A crucial aspect of the origin of pre-mRNA is that codon units are not just added randomly. Instead, the anticodon of pre-tRNA acts as a template to select the matching codon of a premRNA strand. Using the base pairing mechanism, each anticodon of a charged pre-tRNA molecule begins to attract corresponding nucleotides from the prebiotic pool by base pairing (Fig. 7D). After hybridization with anticodons, these triplet nucleotides begin to cluster and link together to form small chains of oligonucleotide with codon bases. Several small oligonucleotide chains begin to link to form a longer strand of a pre-mRNA molecule which becomes a database for storing the information of several amino acids (Fig. 7E). This coded pre-mRNA became the binding partners for pre-tRNA, enhancing mutual stability and instant cognition. This is a turning 
point in the origin of translation when a pre-mRNA molecule becomes a digital strip for the storage of genetic information in a separate device in the nucleotide language. Translation is easier to evolve, logically as well as chemically, if there is already a triplet-amino acid assignment present. Eventually, several strands of pre-mRNA are joined to form a longer strand of pre-mRNA. These pre-mRNA genes are very short, no longer than 30 to 80 nucleotides. The main feature of pre-mRNA is its heterogeneity for information content. A triplet code sequence with random codon assignment has very high information content for protein synthesis. With different combinations of codons and varied lengths of pre-mRNA strand, a wide range of amino acid information could be stored for synthesis of longer protein chain (Fig. 7F).

$<$ Figure 7 about here $>$

With the emergence of pre-mRNA, the information of anticodon assignment of large pretRNA populations can be transferred and stored in a codon message, along the strand of a premRNA molecule. Along the linear strand of a pre-mRNA molecule, digital information for coding amino acids emerged symbiotically with the help of the anticodon of pre-tRNA molecules. Biological information was not only concentrated but also specified along the strand of a pre-mRNA molecule. Charged pre-tRNA becomes the carrier of a specific amino acid that attached to the matching codon of pre-mRNA.

During the interaction of charged pre-tRNA with pre-mRNA, each aminoacyl pre-tRNA (aa-pre-tRNA) molecule transported and selected specific amino acids for protein synthesis. This is how information enters into the codon of pre-mRNA molecule in a storage format for a specific amino acid via the anticodon. This leads to the beginning of the information stage. The information is laid down in the sequences of pre-mRNA, whose quantity is expressed by the 
lengths of those sequences. These base-pairing attachments between charged pre-tRNA and premRNA provided the structural basis for translation.

The aa-pre-tRNA brings this specific amino acid to this pre-mRNA site during translation, where its anticodon binds to the complementary codon. Initially four short oligonucleotides, each with a specific codon, were formed and joined in different combinations, specifying four amino acids such as valine, alanine, aspartic acid, and glycine [69]. This is the first stage of the origin of the primitive genetic code, involving four amino acids, in which a small number of amino acids were coded by a small number of triplets (Fig. 7D). These four amino acids were readily available from the prebiotic vent environment. These oligonucleotides with codons are linked together by random combinations to form a pre-mRNA strand with a coded message (Fig. 7E). Once the base sequence of pre-mRNA is stored for a number of amino acids, a rudimentary translation begins to initiate between pre-tRNA and pre-mRNA to synthesize the protein products that provide some modest catalytic, structural, and binding feature in the RNA/protein world. Most likely, the code assignments and the translation mechanism evolved together [55]. Pre-mRNA molecules, customized by pre-tRNA, multiplied in the vent environment and linked into longer strands of pre-mRNA to become a genetic reservoir, a digital recipe for proteins synthesis. However, at this stage, pre-mRNA can contain limited genetic information for four amino acids or their multiplied combinations.

During the initial translation process, each pre-tRNA carries its corresponding amino acid on its end (Fig. 7F). When a charged pre-tRNA recognizes and binds to its corresponding codon of pre-mRNA, then the growing amino acid chain transfers to the single amino acid of the pretRNA. The pre-tRNA molecule begins to translate the codon of the pre-mRNA molecule in the $5^{\prime}$ to $3^{\prime}$ direction (5' and 3' refer to carbons on sugar subunits of RNA). The codon for the first 
amino acid in the chain (the amino end of the protein) is always at the $5^{\prime}$-end of the pre-mRNA. Likewise, the codon for the last amino acid in the chain is at the $3^{\prime}$-end of the pre-mRNA. As the translation began along the strand of pre-mRNA, the triplet GUC coded for the amino acid valine. An aminoacyl pre-tRNA entered the site where it hybridized the codon. Here, a ribozyme, the precursor to peptidyl transferase of ribosome, performed two critical functions. First, it detached the valine from its pre-tRNA, which was ready to make a growing amino acid chain, and released the pre-tRNA. Second, it catalyzed the formation of a peptide bond between that amino acid and the one attached to the next codon site. The first pre-tRNA, carrying the amino acid glycine, paired with the codon GCC. With the arrival of the second pre-tRNA, carrying valine, the first pre-tRNA, like a runner in a relay race, passed its glycine to the next, linking with valine and was ejected. The third pre-tRNA with anticodon CUC hybridized with the next codon, GAC, bearing the aspartic acid, and picked up the link of glycine and valine. The next step repeats when a new aminoacyl pre-tRNA prepares to attach to the next codon site CGG for alanine. Here it would receive the newly formed polypeptide link of valine-glycine-aspartic acids. To this link, alanine would be added. This is the way a string of bases of pre-mRNA is translated into a sequence of amino acids. The released amino acids chain of valine, glycine, aspartic acid, and alanine, are joined together by a peptide bond to form a newly synthesized protein (Fig. 7F). Ribozymes functioned as a catalyst to break the acyl bond holding the growing amino acid chain on the pre-tRNA, and link the new incoming amino acid to the protein chain by a peptide bond. Those ribozymes involved in the protein synthesis were the precursors for the peptidyl transferase of the larger unit of the ribosomes.

The various synthetic proteins produced through primitive translation accumulated inside the protocells for added protection. The association between amino acids and codons - for 
example between GUC and valine-is called the code. In this way, the genetic code begins to translate in a rudimentary form, as the short chain of proteins is built according to the instruction from the linear order of codons on the pre-mRNA. The process continues until the pre-tRNA molecule reaches the last codon in the pre-mRNA strand. It stops because there are no more codons to match. The completed protein chain is clipped off by the ribozyme. Once the complete protein is made, the pre-tRNA was discarded, and the pre-mRNA was broken down and its nucleotides recycled. The newly synthesized proteins functioned as enzymes for specific catalysis.

This initial code-programming and storage operation of the pre-mRNA by the pre-tRNA must have occurred within the protective environment of the protocells (Fig. 7C). By pairing with the anticodons of the pre-tRNAs, the codons of the pre-mRNA not only selected the appropriate amino acids; they also help to immobilize the pre-tRNAs. To initiate primitive translation, the pre-mRNA strand needed a substrate where pre-tRNA molecules would sequentially bind one codon after another. In the absence of the ribosomes, the inner surface of the membrane would have served as a substrate for holding the pre-mRNA in position for pairing with the anticodon.

The availability of several groups of new enzymes enlarged both the structural and the functional capabilities of the pre-mRNA and pre-tRNA molecules, evolving into the more efficient mRNA and tRNA. This evolutionary transformation was characterized by a progressive refinement of the translation system and an increase of the genetic code. As more and more pretRNA guided pre-mRNA molecules began to emerge, they continuously replicated, increasing their population in the prebiotic pool, linking together in various combinations to form longer strands of mRNA molecules. tRNA and mRNA outnumber their precursors pre-tRNA and pre- 
mRNA through base pairing and replication. These longer mRNA genes arose as replication increased in accuracy. Each mRNA contained about 100 to 200 nucleotides (Fig. 7E).

\subsection{The Origin of Ribosomes}

Translation needs one more piece of the molecular machine to continuously make protein in an assembly line - the ribosome. Ribosomes link amino acids together in the order specified by mRNA molecules. They provide the environment for controlling the interaction between codons of mRNA and anticodons of aminoacyl-tRNA in the creation of proteins. The ribosome can be thought of as a giant conglomerate of RNA and protein molecules, hundreds of times larger than typical enzymes. It is the nexus of codependence between rRNAs and r-proteins. It behaves like a small moving factory that travels along the mRNA template, engaging in rapid cycles of peptide bond synthesis. It represents one of nature's most sophisticated biochemical nanomachines in the cells. The translation of encoded information of mRNA and the linking of amino acids selected by tRNAs are at the heart of the protein production process. Ribosomes can link amino acids together at a rate of 200/minute. Therefore, small proteins can be made fairly quickly. Once a new protein chain is manufactured, the ribosome is released from protein synthesis to enter a pool of free ribosomes that are in equilibrium with separate small and large subunits [57].

The ribosome is composed of two-thirds of RNA and one-third protein. It is made of about 50 ribosomal proteins (r-protein) wrapped up with 4 ribosomal RNAs (rRNA) and is therefore a ribonucleoprotein (Fig. 8). Although ribosomal proteins greatly outnumber ribosomal RNA, the rRNAs account for more than half the mass of the ribosome. A bacterial cell may 
contain as many as 20,000 ribosome complexes, which enable continuous production of several thousand different proteins, both to replace degraded proteins and to make new ones for daughter cells during cell division. A ribosome physically moves along an mRNA strand, reads the codon sequences of mRNA, catalyzes the assembly of amino acids into protein chains using the genetic code. It uses tRNAs to mediate the process of translation from the nucleotide language of mRNA into the amino acid language of proteins with help of various accessory molecules. Each ribosome can bind one mRNA and up to three tRNAs. Central to the development of ribosomes are RNAs that spawn the tRNAs, and a symmetrical region deep within the large ribosomal RNA, where the peptidyl transferase reaction occurs [57, 70-71].

Recent bacterial ribosomes shed light on the origin, evolution, morphology, and composition of primitive ribosome that emerged in the RNA/protein world. The bacteria have smaller ribosomes, termed 70S ribosomes, which are composed of two major subunits of unequal size, called the large (50S) and the small (30S) subunits; each consists of one or two RNA chains and scores of proteins (Fig. 8). The small subunit (SSU) is where mRNA and tRNA molecules interact to read the genetic code, and the large subunit (LSU) is where the growing protein chain is synthesized from the amino acids attached to tRNAs. Thus, the small subunit is mainly decoding mRNA, but the large subunit has mainly a catalytic function. In the large subunit, rRNA performs the function of an enzyme and is termed as a ribozyme. In prokaryotic ribosomes, the small subunit, $30 \mathrm{~S}$, is made of one ribosomal RNA and 21 ribosomal proteins, while the large subunit, 50S, is made of two ribosomal RNAs and 31 ribosomal proteins. The two subunits fit snugly in a slot, through which a strand of the mRNA molecule runs between them, after the fashion of a tape through a cassette player. The ribosome glides through the mRNA tape, which then carries out its instructions bit by bit, linking amino acids together, one 
by one in a specified sequence, until an entire protein has been synthesized. The ribosomal RNAs are programmed to recognize the codon as it appears on mRNA. When the production of a specific protein is finished, the two subunits of ribosome drift apart [70,71]. Ribosomes have only a temporary existence. The large and small subunits of the ribosome undergo a cycle of association and dissociation during each round of translation. Similarly, once the protein is made, mRNA is broken down and the nucleotides recycled.

$<$ Figure 8 about here $>$

The ribosome evolved prior to the emergence of DNA and the cellular life in the RNA/protein world. Ribosome evolution is intricately linked to the prior evolution of mRNA, tRNA, and primitive form of the genetic code and translation. The origins and evolution of ribosomes remain printed in the biochemistry of extant life and in the structure of the ribosome. Most theories propose that the ribosome was a functional takeover of a primitive RNA-based translation system in a coordinated series of chemical reactions. RNA is thought to be responsible for the bulk of a ribosome's work. Recent structures of ribosomes have shown unambiguously that the essential functions of the ribosome, such as decoding, peptidyl transfer, and translocation, all appear to be mediated by RNA [72]. Harish and Caetano-Anolles [22] documented how accretionary tRNA building blocks gave rise to functional rRNA. The decoding center where mRNA is located in the small subunit and is primarily formed from $16 \mathrm{~S}$ rRNA. The rRNAs are folded into highly compact, precise three-dimensional structures that form the core of the ribosome. The rRNAs give the ribosome its overall shape. Thus, the widely popular concept of 'the ribosome is a ribozyme' was born; ribozymes must have preceded coded protein synthesis [41]. 
In recent times, the role of proteins in the origin of ribosomes is gaining currency, implying that the ribosome may have first originated in an RNA/protein world where both amino acids and a variety of enzymes were available in the hydrothermal crater vent environment $[4,11,21,22,73]$. Ribosomal proteins are not passive contributors to ribosome function. They are generally located on the surface, where they fill the gaps and crevices of the folded rRNA. The main role of the ribosomal proteins seems to fold and stabilize the rRNA core, while permitting the changes in rRNA conformation that are necessary for this RNA to catalyze efficient protein synthesis. The ribosomal proteins provide the structural framework for the $23 \mathrm{~S}$ rRNA which actually carries out the peptidyl transferase reaction. In the absence of ribosomal proteins $23 \mathrm{~S}$ rRNA is unable to serve as a peptidyl transferase activity. Assembly of large and small subunits dependents upon ribosomal proteins [21,73]. Several ribosomal proteins assist in the assembly of the large subunit by providing unstructured, highly positively charged protein sequences that bind amino RNA segments together and extend to the center of the subunit [74]. These extensions fold cooperatively with ribosomal proteins to produce the small subunit.

Why would an RNA structure evolve to make proteins if the protein did not already exist that would confer a selective advantage on the ribosomes capable of synthesizing them? The availability of even simple proteins could have significantly enlarged the otherwise limited catalytic function of RNA. Many prebiotic protein enzymes carried out several key functions in the primitive translation system. Moreover, before the origin of ribosome, the production of simple proteins had already commenced through the interactions of mRNA/tRNA/aaRS (Fig. 7). Perhaps ribosomal proteins were synthesized during the primitive translation system, which were then recruited to build the ribosome step-by-step. RNAs and proteins developed a symbiotic relationship to create ribosomes in the RNA/protein world [21]. These r-proteins took an active 
part in stabilizing the evolving ribosomes and in interacting with many rRNA sequences. Because the number of proteins greatly exceeded the number of RNA domains, it can hardly come as a surprise that every rRNA domain interacted with multiple proteins in ribosomes [74]. Ribosomes are not entirely ribozymes, but more accurately ribonucleoprotein (RNP), a complex that can have as many as 62 r-proteins with only 3 rRNA molecules (Fig. 8). Virtually all rproteins are in contact with the rRNA. So, it makes sense that this assemblage is a result of a long and complicated process of gradual coevolution of rRNAs and r-proteins. Both the assembly and synthesis of the ribosomal components must occur in a highly coordinated fashion [22]. Their phylogenetic analysis reveals that the ribosomal protein/rRNA coevolution manifested throughout the prebiotic synthesis process, but the oldest protein (S12, S17, S9, L3) appeared together with the oldest rRNA substructures that were responsible for both the decoding and ribosomal dynamics 3.3-3.4 Ga. Although protein synthesis is largely carried out by different kinds of RNA molecules within the ribosome such as mRNA, tRNA, rRNA, and peptidyl transferase, aminoacyl synthetases (aaRS) played a crucial role as a protein enzyme that attached the appropriate amino acid onto its tRNA during protein synthesis. The synthetases is equal in importance to the tRNAs in the decoding process because it is the combined action of synthetases and tRNAs that allows each codon in the mRNA molecule to associate with its proper amino acid. The synthetases could not emerge in a pure RNA world. Similarly, both rRNA and the 50S subunit proteins are necessary for the peptidyl transferase activity during the peptide bond formation, but the actual act of catalysis is a property of the ribosomal RNA of the larger subunit (Fig. 8). The cumulative conclusion that seems to be most in accord with biochemical evidence is that the RNA/protein world preceded ribosome. 
The accretion model describes the origin and evolution of ribosomes [22]. Given that the ribosome is quite ancient, it is likely that rRNAs and r-proteins coevolved to build this complex nanomachine. Ribosomes, like the rings of a tree, contain the record of their history, spanning 4 billion years. Like rings in the trunk of a tree, the ribosome contains components that functioned on in its early history. It accreted to grow bigger and bigger over time. But the older parts froze after they accreted, like the rings of a tree (Fig. 8). Recent phylogenetic work on ribosomal history suggests that both RNAs and proteins contributed to the formation of the ribosome core through accretion, recursively adding expanding segments [22, 23]. Ribosomes contains life's most ancient and abundant polymers, the oldest fragments of RNA and protein molecules. It most likely a molecular relic of the RNA/protein world [4].

Both ribosomal subunits have separate functions. Peptide bond formation occurs at the peptidyl transferase center (PTC) of the large subunit, whereas mRNA sequences are decoded on the small subunit. mRNA decoding contributes to the specificity of protein synthesis on the ribosome. In isolation, both subunits can perform their respective functions (Fig. 8). By itself, the large subunit will catalyze the formation of peptide bonds between aminoacyl-tRNA-like substrates. By itself, the small subunit binds mRNA, and when mRNA is bound, it will bind tRNAs in a codon-specific manner. In an RNA world scenario, the ribosome originated in the peptidyl transferase center of the large ribosomal subunit $[75,76]$. There are no r-proteins close to the reaction site for protein synthesis. This suggests that the protein components of the ribosome do not directly participate in the peptide bond formation catalysis, but rather the proteins act as a scaffold that may enhance the ability of rRNA to synthesize protein. Ribosomes themselves, although fundamentally ribozymes in nature, still require r-proteins to fold their 
rRNAs into biologically active conformations and to optimize the speed and accuracy of their functions [70]. The ribosomal surface is an integrated patchwork of rRNAs and r-proteins.

Currently, there is a debate about the origin of the ribosomal subunits: which unit came first, the small or the large subunit? It is likely that the PTC of large ribosomal subunit evolved from pre-tRNA molecules by duplication of the minihelix [61]. In this view, the simple function of peptide bond formation at the PTC site came first, and the specifications based on the codon sequence came later. In other words, the large subunit of the ribosome came first, followed by the addition of the small unit. However, these proposals do not link the protein synthesis to RNA recognition and do not use a phylogenetic comparative framework to study ribosomal evolution.

A contrasting view of the origin of ribosomal subunits has been proposed by other authors who favor the small unit of ribosome as the first. Harish and Caetano-Anolles [22] studied the phylogeny of ribosome and reconstructed its likely history. They found that components of the small ribosomal subunit evolved earlier than the catalytic peptidyl transferase center of the large ribosomal subunit. They suggested that ribosomal RNA and proteins coevolved tightly, starting with the oldest proteins (S12 and S17) and the oldest rRNA helix in the small subunit (the ribosomal ratchet responsible for ribosomal dynamics) ending with the modern multi-subunit ribosome. They hypothesized a major transition in the evolution of ribosomes around $4 \mathrm{Ga}$ brought independently evolving subunits together by infolding intersubunit contacts and interaction with full cloverleaf tRNA structures.

In our view, both the small subunit and the large subunit of the ribosome appeared simultaneously and worked together, because the decoding of mRNA and the peptide bond formation were both essential components during protein synthesis. These two subunits might have coevolved to join during translation and separate after protein synthesis. The rRNAs are 
folded into highly compact, precise three-dimensional structures to form the core of the ribosome, whereas r-proteins are generally located on the surface, where they fill the gaps and crevices of the folded RNA and act to fold and stabilize the core [74]. As these two subunits expanded through accretion, eventually arriving at the size of the bacterial ribosome, accretion stopped, they then bound together during protein synthesis, and finally spilt apart when the ribosome finished reading its mRNA molecule (Fig. 8).

If the fundamental functions of the ribosome are based on rRNA, why are there so many ribosomal proteins, some of which are highly conserved? One explanation is the rRNA does not fold into its functional state in the absence of r-proteins. Another reason for the presence of proteins in ribosomes is that they improve the efficiency and accuracy of translation [75]. Both rRNAs and r-proteins work cooperatively in ribosomes to perform the multitask of protein synthesis. Harish and Caetano-Anolles suggested that functionally important and conserved regions of the ribosome were recruited and could be relics of an ancient RNA/protein world [22]. The corollary is that a fully functional biosynthetic mechanism responsible for primordial peptides and ancient r-proteins must have existed that in time was superseded by the ribosome.

According to this accretionary model, very early in ribosomal evolution, rRNA helices interacted with r-proteins to progressively form a core which mediated nucleotide interactions, and later served as the center for the coordinated and balanced RNP (ribonucleoprotein) accretion that evolved into our modern ribosomal function. The early existence of smaller functional units of ribosome, capable of carrying out different translational steps such as peptidyl transferase, decoding, and aminoacylation, along with the development of A, P, and E sites for the positioning of tRNA molecules, can be inferred from the phylogeny. These small functional RNA/protein units were incrementally accreted and refined by the incorporation of additional 
rRNA and r-protein molecules. Similarly, Nissen et al. [77] provide the first atomic resolution of the larger of the two subunits of the ribosome. From this structure, they deduce that the RNA components of the large subunit accomplish the key peptidyl transferase reaction. Thus, rRNA does not exist as the framework to organize catalytic proteins. Instead, the proteins are the structural units and they help to organize the key ribozyme. A 'pure' RNA world is incompatible with the existence of the coevolutionary pattern proposed for ribosomal molecules.

Perhaps rRNA, such as noncoding ribozymes, acquired amino acids as cofactors making them more efficient catalysts. By using cofactors, the range and specificity of catalytic activity can be increased. Ribozymes would have been in greater need of cofactors than protein enzymes, because, without them, the range of reactions they can catalyze is much smaller [51].

In our endosymbiotic model, rRNAs and r-proteins were brought into close proximity within the plasma membrane to form the building block of the primordial ribosome. The origin of the ribosome precursor by fusion and the accretion of the key components of these ribosomal RNA and protein molecules is the likely scenario. rRNA and r-protein molecules began to fuse because of a chiral preference and formed the rudimentary ribosomes. Once the core of the ribosome formed, mRNA and tRNA molecules were recruited to help in translation through a trial and error method. Once a true mRNA, and the core small subunit of ribosome were in place, the ribosome would become increasingly complex by adding early conserved rRNA and rproteins. Ribosomal proteins played an important role in supporting the ribosome structure and in promoting translation. With the onset of operational coding, tRNA began to assemble amino acids into long chains of proteins. Here we suggest that a ribosome-like entity was one of the key intermediates between prebiotic and cellular evolution, formed by endosymbiosis and the fusion 
of rRNA and r-protein molecules. Once ribosomes were installed inside the protocell membranes, the translation system was greatly improved.

In vitro constructions of ribosomes can shed new light on the mechanism of protein synthesis and provide deeper insights into the way nature has assembled this complex machine. Working with E. coli cells, Orelle et al. [78] combined natural ribosomal proteins with synthetically made rRNA, which self-assembled in vitro to create semi-synthetic, functional ribosomes. Comprising 57 parts — three strands of rRNAs and 54 proteins — an artificial ribosome (termed Ribo-T) in which two subunits are tethered together by a short length of RNA is able to carry out normal translation and pump out custom-made proteins. The ability to make ribosomes in vitro is a process that mimics nature and opens up new avenues for the study of ribosome synthesis, suggesting the coevolution of ribosomal RNAs and proteins.

\subsection{Protein Synthesis}

We have now reviewed the emergence of all the major components of the translation machinery for protein synthesis. Translation of the mRNA template converts nucleotide-based genetic information into the 'language' of amino acids to create a protein product. Translation requires the input of an mRNA template, tRNAs, aminoacyl-tRNA synthetases, ribosomes, and various enzymatic factors. The tRNAs functions as the adaptor molecules that transport amino acids to ribosomes in response to codons in mRNAs, where peptidyl transferase catalyzes the addition of amino acid residues to the growing protein chain in protein synthesis by means of peptide bonds. The ribosomes serve as the sites for protein synthesis, and link amino acids 
together in the order specified by mRNA. They always translate the mRNA from the $5^{\prime}$ to the $3^{\prime}$ direction like a sliding machine.

Proteins have a modular chemical structure that allows the construction of widely different molecular machines using the same basic set of amino acids, each with a different size and chemical character. Protein synthesis requires the concerted effort of dozens of different enzymes. 20 tRNA molecules, each with their own dedicated synthetase enzyme, are built for 20 amino acids. Modern protein synthesis proceeds with the participation of 20 amino acids, tRNA, mRNA, ribosomes, various enzymes including aminoacyl-tRNA synthetase, ribozymes, peptidyl transferase, and a considerable number of proteinous factors, ATP, GTP, etc. More than 120 species of RNAs and proteins are involved in the process of protein synthesis [47]. These biomolecules in the hydrothermal vent were related, encapsulated, and interacted with each other in complex ways, like an autopoietic machine. Yet the whole series of molecules in the translation process functioned with astounding precision, in a kind of molecular choreography, that gave birth to the universal genetic code.

The translation, the synthesis of proteins from mRNA templates, is well-known, and will be briefly reviewed here [57]. In the ribosome, there are three stages and three operational sites involved in the protein production line, all work in harmony. During the initiation stage, a small ribosome subunit links onto the 'start end' of an mRNA strand. Aminoacyl-tRNA also enters site A of the ribosome. The production of protein has now been initiated. The second stage, elongation, consists of joining amino acids to the growing protein chain, according to the sequence specified by the message. The incorporation of each amino acid occurs by the same mechanism. In the termination stage, the ribosome reaches the end of the mRNA strand, a 
terminal or 'end of the protein code' message. This registers the end of production for the particular protein coded by this strand of mRNA.

Four binding sites are located on the ribosome, one for mRNA and three for tRNA (Fig. 8B). The three operational sites are A (acceptor), P (peptidyl), and E (exit), (reading from the mRNA entry site, conventionally the right-hand site) (Fig. 9B). The translation of mRNA begins with the formation of an initiation complex, which consists of a charged tRNA bearing methionine; a small subunit bound to mRNA triggers the translation process. The next charged tRNA enters the A-site, peptidyl-tRNA is bound to the P-site, while deacylated tRNA exits via the E-site. The ribosome moves along the mRNA one codon at a time in the $5^{\prime}$-to 3 ' direction. An amino acid is added to the protein chain by transferring the protein from peptidyl-tRNA in the P-site to aminoacyl-tRNA in the A-site. Translation takes place in a four-step cycle. In step 1, an oncoming aminoacyl-tRNA with the appropriate anticodon enters the vacant A-site where it hybridizes with the appropriate codon of an mRNA molecule by hydrogen bonding (Fig. 8C). In step 2, its amino acid is then linked to the protein chain held by the tRNA in the neighboring P site (Fig. 9D). Here amino acids are joined together by a peptide bond that links the carboxyl end $(\mathrm{COOH})$ of one amino acid to the amino end $\left(\mathrm{NH}_{2}\right)$ of a second amino acid of the tRNA at the Asite.

At the center of the large subunit of the ribosome lies the enzyme peptidyl transferase, a ribozyme, that catalyzes the addition of amino acids at each successive step of the growing protein assembly in protein synthesis by means of peptide bonds. This reaction is catalyzed in two steps: it breaks the bond between the tRNA in the P-site and its amino acid; it catalyzes the formation of a peptide bond between that amino acid and the one attached to the tRNA in the Asite. The peptide bond formation takes place through reaction between the peptidyl-tRNA in the 
P-site, and the amino acid of the aminoacyl-tRNA in the A-site, where the growing protein chain is transferred. Thus, a ribosome can carry two aminoacyl-tRNAs simultaneously: its P site is occupied by a peptidyl-tRNA, which carries the protein chain so far synthesized, while the A site is used for entry by an aminoacyl-tRNA carrying the next amino acid to be added to the chain. In step 3, the ribosome moves one triplet along the mRNA in the 3 'direction (Fig. 8E). The movement transfers the empty (deacylated) tRNA out of the P-site, while a new peptidyl-tRNA moves into the P-site. In step 4, the next codon to be translated now lies in the A-site, ready for a new aminoacyl-tRNA to enter, where the new cycle would be repeated (Fig. 8F). The presence of a stop codon at the A site of the ribosome terminates translation. Finally, the two subunits of the ribosome separate when the synthesis of the protein is finished (Fig. 8G). The translation machinery is helped along by two elongation factors, known as EF-1 and EF-2. These factors accelerate the process by supplying energy to the ribosome for the many functions it has to perform [57].

$<$ Figure 9 about here $>$

The binding of tRNA to its $\mathrm{A}, \mathrm{P}$, and $\mathrm{E}$ sites in the ribosome allows the positioning of the substrates for the catalytic step for translational accuracy, which was lacking in the mRNA/tRNA moieties. In its function as a decoder of mRNA, the ribosome utilizes the differences in specific binding energy between cognate and noncognate tRNAs. Conformation changes of the ribosome and the tRNA play a crucial role in determining decoding accuracy. For fast and accurate recognition of the appropriate tRNA, the ribosome utilizes large conformational proofreading [79]. The independent but coordinated functions of two subunits of ribosomes, including their ability to associate at initiation, rotate during elongation, and dissociate after protein release, are 
an established model of protein synthesis [72]. Protein synthesis is an expensive process. ATP is used to provide energy at several stages, including the charging of tRNA with amino acid.

Once released from the ribosome, the long chain of amino acids will fold spontaneously in intricate contortions. A protein has been formed. It folds into functional three-dimensional structure and proper characteristic shape: some parts form sheets, while others stack, curl, and twist into spirals. The sequence of amino acids determines the shape and conformation of a protein and, thereby, all its physical and chemical properties. A protein molecule folds spontaneously during or after biosynthesis, but the folding process depends on the solvent, the concentration of salts, the temperature, the possible presence of cofactors, and the molecular chaperons [80]. Proteins must fold in specific ways to function properly.

\section{The Origin and Evolution of the Genetic Code}

The genetic code is the universal language of life. It defines the rules by which information stored in mRNA sequences is translated into the corresponding amino acids sequences to proteins. The genetic code is universal; it is the same for all organisms, from simple bacteria to eukaryotes to animals to humans. The genetic code maps the 3-letter words, in a 4letter alphabet, of the mRNA language $\left(4^{3}=64\right.$ codons $)$ to the protein language alphabet of 20 amino acids. Before focusing on the origin of the code, let us consider its most important properties. The universal genetic code consists of 64 codons that specify 20 amino acids, and start and stop sites. The large number of codons is due to redundancy in the code; that is, several codons may specify the same amino acids. All but two of the amino acids (methionine and tryptophan) have more than one codon, many have two, one has three, several have four, and two 
of them have six codons (Table 1). The amino acids that are used more often in proteins are specified by a greater number of different codons. No codon goes unused. The genetic code has redundancy, but no ambiguity. For example, although codons GAU and GAC both specify aspartic acid (redundancy), neither of them specifies any other amino acid (ambiguity). The genetic code is nonoverlapping, meaning the 'words' follow each other without gaps or overlaps. Each codon in mRNA specifies one amino acid in the protein product. The code is also commafree. There are no commas or other forms of punctuation within the coding regions of mRNA molecules. During the translation, the codons are read consecutively. The code is ordered. Multiple codons for a given amino acid and codons for amino acids with similar chemical properties are closely related, usually differing by a single nucleotide $[46,47]$. The arrangement of the genetic code is distinctly non-random and is such that neighboring codons are assigned to amino acids, with similar physical properties. Hence the effects of translation error are minimized with respect to reshuffled codes. The digital information in the linear sequence of nucleotides in mRNA is translated into analog sequences of amino acids in proteins according to the genetic code [45]. The vast majority of living organisms follow the same universal genetic code. The most important exceptions to the universality of the code occurs in the mitochondria of mammals, yeast, and several other species.

The table of universal (or standard) genetic code, showing the association of each threeletter code to its respective amino acid, is a little dictionary, a Rosetta stone, just as Morse code relates the language of dots and dashes to the twenty-six letters of the alphabet (Table 1). The very existence of two languages (with the code being a translational intermediary) implies a directional course of evolution. The table is not a random accident, it is the result of very specific selection. The mRNA is a linear polymer of four different nucleotides and is read consecutively 
in groups of three nucleotides (codons) to form the 'words' of the message without any comma. This is known as an 'open reading frame.' Every sequence in mRNA can be read in its $5^{\prime} \rightarrow 3^{\prime}$ direction in three reading frames. Each 3-base codon stands for a single amino acid, so there are 64 possible combinations of three nucleotides. The arrangement of the codons in the universal code is highly nonrandom. The code has been confirmed by several experimental methods $[53,80]$.

$<$ Table 1 about here $>$

The origin of the genetic code remains elusive, even though the full codon catalog was deciphered over 50 years ago [81]. It's still not clear why the genetic code might have originated in the prebiotic world leading to the information age. Perhaps critical biomolecules had evolved in the vent environment in the chemical stage and began to attract each other. A stereochemical relation between some amino acids and cognate anticodons/codons is likely to have been an important factor in the origin of the genetic code. The biosynthetic relationships between amino acids and RNAs are closely linked to the organization of the genetic code. We have suggested that the transition from the noncoded to the coded protein might have resulted in the origin of the code. How accurately the genetic code is translated depends on two-steps in protein synthesis: precise decoding of mRNAs and accurate synthesis of aminoacyl-tRNAs. aa-tRNAs are made by aaRSs, which match specific amino acids with the corresponding tRNAs as defined by the genetic code. Thus, the crucial feature of the genetic code is the attachment of particular amino acids to tRNA molecules, a step carried out by assignment enzymes such as aminoacyl-tRNA synthetase. We are suggesting this attachment first occurred between pre-tRNA and specific amino acid, and was carried out by pre-aaRS enzyme. Since various enzymes were available in the RNA/protein world, we propose that the functional enzyme for binding an amino acid to its 
corresponding tRNA was pre-aaRS from the beginning, not ribozyme, as previously suggested by other workers [51].

Although we know which codon encodes which amino acid, we do not know why the specific codon assignments take their actual form. Why are there exactly four nucleobases in mRNA? Why does life use 20 amino acids for making proteins, when 70 amino acids were available in the hydrothermal vents from the cosmic source [49]? If the code evolved at a very early stage in the history of biosynthesis, perhaps during its prebiotic phase, the four nucleotides in mRNA and 20 amino acids in proteins may have been the most promising case for optimization by natural selection for chemical reactions relevant at that stage. Perhaps it is simply a 'frozen accident,' a random choice that just locked itself in, and remained, mostly, unchanged once the optimal design was reached [35]. Any change of codon reassignment may be lethal because it would trigger mutation, which would be dispersed throughout all proteins in the cell. This accounts for the fact that the code is universal in all organisms from bacteria to humans. To account for the sameness in all organisms one must assume that all life evolved from the last universal common ancestor (LUCA). Since then, the universal code remains unchanged for the last four billion years.

\subsection{Origin of the Genetic Code}

Although multiple hypotheses have been proposed to explain why codons are selectively assigned to specific amino acids, empirical data is extremely rare, and difficult to obtain, leaving many theories in the realm of conjecture. Three main concepts on the origin and evolution of the genetic code are: 
(1) stereochemical theory, according to which codon assignments are dictated by physico-chemical affinity between amino acids and the cognate anticodons or codons; perhaps, tRNA molecules matched their corresponding amino acids by their stereochemical affinity [55, 82-84]. Simply put, the hypothesis proposes that symbols in the genetic code (anticodons or codons) may directly bind to the objects (amino acids) they stand for.

(2) coevolutionary theory, which suggests that the code structure coevolved with the amino acid biosynthesis pathways $[67,85]$. This theory suggests that the genetic code is primarily an imprint of the biosynthetic pathways forming amino acids. There are two generations of amino acids: the ten primary or primitive amino acids were formed under prebiotic condition; they serve as a starting point for the synthesis of the remaining ten amino acids which derived from the first set. What happened afterwards, is that some primitive systems evolved the ability to manufacture the secondary amino acids, and eventually also the primary amino acids.

(3) adaptive theory, which postulates that the structure of the code was shaped under selective forces that made the code maximally robust, usually some kind of error minimization [57].

Many other models have emerged as addendums to one of the main models or as some form of hybrid. We believe that these three theories are not mutually exclusive and are compatible with the RNA/protein world because aaRS play a crucial role in translation. Without aaRS, however, tRNA molecules could not be matched with their corresponding amino acids. It has long been conjectured that the universal genetic code (Table 1) evolved from a simpler primordial form that encoded fewer amino acids [35]. The earliest proteins started with 10 amino acids, which have been produced in prebiotic chemistry experiments. Any model for 
the evolution of an early code and translation apparatus in the pre-DNA stage will have to provide conditions that allow tRNA and mRNA for various enzyme factors not only to coexist but also to grow coherently and to evolve optimal function. Our proposed biochemical pathways for the origin of the translation favor three distinct phases for the origin of the genetic code. The early stage of coding might have been initiated in an RNA/protein world by stereochemical interactions between pre-tRNA and amino acids leading to the birth of pre-mRNA molecules for storing genetic information. The activating enzymes were pre-aaRS, precursors to modern aminoacyl-tRNA synthetases, which bound specific amino acid to corresponding pre-tRNA molecules. Subsequently, the code expanded with the involvement of more amino acid-tRNA ligations by aaRS, and the progressive elongation of the mRNA strand for accommodating more and more genetic information. Finally, the code further expanded for redundancy and was optimized through codon reassignment with the emergence of ribosomes, which bound mRNA and tRNA to synthesize proteins (Table 1).

The stereochemical hypothesis postulates that the structure of the code is determined by a physico-chemical affinity between amino acids and cognate anticodons or codons [55, 82-84]. The close linkage between the physical properties of amino acids and tRNA molecules was likely an essential step for the origin of code. A stereochemical relation between some amino acids and cognate anticodons/codons is likely to have been a significant influence in the earliest assignments [87]. It is possible that the chiral D-sugars in RNA attracted the chiral L-amino acids as a stereo pair. Rodin et al. [88] have published an exhaustive analysis of the stereochemical concept and concluded that the genetic code originated before translation. The stereochemical theory is supported by RNA aptamer experiments, in which RNA molecules evolved to bind specific amino acids [84]. Such experiments have provided critical empirical 
data, demonstrating the association of codon triplets with amino acids. Johnson and Wang [89] found that anticodons are selectively enriched near their respective amino acids in ribosomal structure and such enrichment is correlated with the universal genetic code. Ribosomal anticodon-amino acid enrichment reveals that specific codons were reassigned during code evolution. These authors concluded that anticodon-amino acid interactions shaped the evolution of the genetic code.

The nucleobases of mRNA could not make direct bonds with a hydrophobic amino acid side group. tRNA serves as the nucleic acid decoding device that reads the triplet genetic code of mRNA and causes the insertion of codon specific amino acids in a growing protein chain during the process of translation. The specific coding between codon and amino acids takes place in a two-step process via tRNA. For each amino acid, there is a corresponding tRNA molecule for which it has the intrinsic affinity. tRNA molecules function as adaptors by mediating the incorporation of proper amino acids into proteins in response to specific nucleotide sequences in mRNA. The amino acids are attached to the correct tRNA molecules by a set of activating enzymes, aminoacyl-tRNA synthetases. The aaRS recognize, on the one hand, individual amino acids, which they activate via conjunction with AMP, and on the other hand, the cognate tRNA molecules to which aaRS transfer the amino acid residues [89]. The 20 aminoacyl-tRNA synthetases establish the genetic code through aminoacylation reactions that link specific amino acids to tRNAs that bear triplet anticodons. Therefore, at the biochemical level, the genetic code is established by the aaRS, which is one of the oldest protein families, emerging with the advent of the information stage.

Since all tRNAs have similar structures, the identification must take place on a sequence level in combination with subtle structural variations. In most known cases, the anticodon bases 
are part of this set of identity elements. In E. coli, 17 out of 20 amino acids are recognized by anticodons [90]. In a minority of cases, position 73 in the acceptor stem is used to identity amino acids. Its matching codon is also recognized as data, the information of a specific amino acid. tRNA-codon recognition has always been assumed to be result of base-pairing. Anticodon-codon pairing might have initiated the first primitive translation.

It is generally believed that the linking of amino acids to tRNAs played a crucial role for the origin of coding and translation. The original amino acid-binding motifs could have been the actual anticodons of tRNAs. Several authors have proposed that abiotic tRNA molecules could have bound some abiotic amino acids to either improve stability, or to expand their functional capabilities, or both $[70,87,91]$. Without this initial amino acid binding site, it is difficult to see how else tRNA molecules could have become involved with coding specific amino acids. tRNAamino acid pairing interactions were a prelude to the code. Thus, our first clue to the origin of the code is to decipher how primordial tRNAs and amino acids were related by molecular recognition and chemical principles [92].

In contrast to stereochemical hypothesis, the coevolution theory suggests that the original genetic code specified a small number of abiotic simple amino acids, and that, as more complex amino acids were synthesized from these precursors, some codons that encoded a precursor were ceded to its more complex products. The coevolution theory was championed by Wong $[67,68,93]$ and expanded by Di Giulio [95]. It proposes that primordial proteins consisted only of those amino acids readily obtainable from the prebiotic environment, representing about half the twenty amino acids of today, and the missing amino acids entered the system as the code expanded along the pathways of amino acid biosynthesis. The coevolution theory postulates that prebiotic synthesis could not produce 20 modern amino acids, so a subset of the amino acids had 
to be produced through biosynthetic pathways before they could be opted for expanded genetic code and translation $[56,85]$. Wong [86] has defined two types of amino acids depending on whether they were supplied by the hydrothermal vent (Phase 1) or were biosynthetically produced (Phase 2). The first phase of amino acids consists of glycine, alanine, serine, aspartic acid, glutamic acid, valine, leucine, isoleucine, proline, and threonine. Phase 2 amino acids include phenylalanine, tyrosine, arginine, histidine, tryptophan, asparagine, glutamine, lysine, cysteine, and methionine. The first phase of amino acids naturally emerged through prebiotic synthesis in the vent environment, before the emergence of ribosomes. They have been identified in meteorites. The ranks of amino acids in this list strongly correlate with the free energy available in the vent environment for their syntheses: the most thermodynamically efficient are on the top of the list. These 10 amino acids are considered old and were represented in the first stage of protein synthesis. They would play important roles in the primitive GNC-SNS code (Fig. 10). Phase 2, the amino acids entered the code by means of biosynthesis from the Phase 1 amino acids with the emergence of tRNA molecules, aminoacyl transferase enzyme, and ribosomes $[56,67,93]$.

\subsection{Early Stage of Code Evolution: GNC Code}

Eigen and Schuster [94] suggested that in the early phase of the evolution of the genetic code, it is characterized by low fidelities of replication and translation as well as by an initially low abundance of efficiently replicating units. Hypercyclic organization offers multiple advantages over any other kind of structural organization. This hypercycle model can be built to provide realistic precursors such as pre-tRNA and pre-mRNA. The interaction between pre- 
mRNA and pre-tRNA molecules is the beginning of the first stage of the biosynthesis of the templated protein chain, encoded in pre-mRNA. These new generations of amino acids are not only template directed but sequence-directed [65]. Here we propose that the interaction between pre-tRNA and amino acids led to the development of the pre-mRNA strand and the primitive GNC genetic code $[69,95]$.

A primordial code must have a certain frame structure, a grammar of rules, otherwise message cannot be read consistently. The GNC hypothesis refers to the origin of genes. It suggests the universal genetic code originated from a primitive four-amino acid system encoding GADV proteins [69]. The GNC codons include four codons (GGC, GCC, GAC, GUC), which code four GAVD amino acids (glycine, alanine, valine, and aspartic acid). Each letter of GNC represents the following nucleotides: $\mathrm{G}=\mathrm{G} ; \mathrm{N}=\mathrm{A}, \mathrm{U}, \mathrm{C}, \mathrm{G}$; and $\mathrm{C}=\mathrm{C}$. The GADV-protein world is a hypothetical stage of abiogenesis. The GNC code defines the very earliest phases of the genetic code origin, reflecting biosynthetic relationships between four amino acids and four codons. Perhaps GNC code was promoted by the pre-tRNA/pre-mRNA interaction and coevolution [65] (Fig. 7A). As amino acids overtook more and more catalytic duties, the genetic information established so far had to be rewritten, a translation into the language of amino acids by specific interaction was inevitable. The translation required a mini dictionary of nucleotideto-amino acid equivalence hence this was the inevitable moment for the genetic code to emerge. To perform protein translation an elaborate machinery of specialized enzymes is necessary. This machinery must be produced step-by-step before translation can take place at all (Fig. 7D). It seems reasonable to start this process in simplified form using only restricted set of amino acids such as glycine, alanine, aspartic acid, and valine that were of prebiotic origin [54]. 


\subsection{Transitional Stage of Code Evolution: SNS Code}

GNC code evolved into the second generation of the genetic code, called an SNS type where $\mathrm{N}$ arbitrarily denotes any four RNA bases, and S denotes guanine (G) and cytosine (S) $[69,95]$. SNS is composed of 10 amino acids (glycine, alanine, aspartic acid, valine, glutamic acid, leucine, proline, histidine, glutamine and arginine) and 16 codons (GGC, GGG, GCC, GCG, GAC, GAG, GUC, GUG, CUC, GUG, CCC, CGC, CAC, CAG, CGC and CGG). The SNS type code shares similarity with the Phase 1 amino acids generated in prebiotic synthesis [55]. The remaining ten amino acids are derivatives of the first ten primitive amino acids. Support for the GNC-SNS primitive genetic code hypothesis comes from the following six indices: hydropathy, -helix, $\beta$-sheet and $\beta$-turn formabilities, acidic amino acid content and basic amino acid content (Table 1). This early genetic code continued to evolve, maximizing its efficiency, until it arrived at its current state, the universal code. This universal code had the edge over the GNC-SNS primitive code, reliability wise, so natural selection would favor it, and, by process of successive refinement, an optimal code would be reached. The universal code is the optimization of functional efficiency to minimize error during translation.

\subsection{Final Stage of Code Evolution: Universal Genetic Code}

The present genetic code is most probably the outcome of a long selective process in which many different codes were tested against each other. As more and more biotic amino acids were synthesized and available in the vent environments, more complex molecules such as tRNA, mRNA, and ribosomes emerged and produced Phase 2 amino acids. At this stage, the 
universal code began to appear (Table 1). A direct correlation has been found between the hydrophobicity ranking of most amino acids and their anticodons. In this stage, ribosomes emerged to facilitate high-fidelity translation. tRNA assigned more codons to mRNA, this led to the emergence of the universal genetic code with 64 codons specifying 20 amino acids [88]. The driving force during this process is not only to minimize translation error but positive selection for the increased diversity and functionality of proteins which are made with a larger amino acid alphabet. With 64 codons, the strand of mRNA became longer, forming a continuous sequence with the start and stop sites for protein synthesis. In the 'codon capture theory,' the number of encoded amino acids is kept constant and equal to 20 , and the coding codons change in the evolution, a key role played by the anticodon $[84,92]$.

Ikehara [69] hinted that the universal genetic code with 64 codons originated from the SNS code which allowed redundancy. Four codon assignments, corresponding to tyrosine, tryptophan, serine, and isoleucine, were newcomers from the SNS code, suggesting that these amino acids are later additions to the code [68]. This idea is consistent with view that these four amino acids are later additions of code. Undoubtedly there were many experiments with a variety of coding methods before adopting the current system in which 61 codons specify 20 amino acids and 3 additional codons for the start and stop sites.

The code is obviously not the result of a random assignment of codons to amino acids. It has a structure. Synonyms are grouped. The large number of codons is due to redundancy in the code; that is, several codons may specify the same amino acids (Table 1). Some generalizations can be made about redundancy of the code. For example, similar codons specify the same amino acids to reduce harmful effects of mutation. For example, GUU, GUC, GUA, and GUG all specify valine. Similarly, amino acids that are used more often in proteins are specified by a 
greater number of different codons. For example, the commonest amino acid, leucine, is coded by six codons (UUA, UUG, CUU, CUC, CUA, and CUG), and the relatively rare tryptophan by one codon (UGG) [51] (Table 1). The expanded genetic code is so universal that there is strong evidence that all life on Earth had a single origin in the universal code before the last universal common ancestor (LUCA) evolved.

Between the codon and anticodon, there is a paradox in the expanded genetic code. The 20 amino acids found in proteins are specified by 61 different mRNA codons. Instead of containing 61 different tRNAs with 61 different codons, though, most bacterial cells contain some 30-40 different tRNAs. Consequently, many amino acids have more than one tRNA to which they can attach; in addition, many tRNAs can pair with more than one codon [57].

Although some features of the expanded code may reflect the early version, there are others that appear adaptive. The genetic code has certain regularities and structures [97]. There is a strong correlation between the first bases of codons and the biosynthetic pathways of the amino acid they encode. The first letter of the codon is allied to the precursor of the amino acid. The second letter signifies whether an amino acid is soluble or insoluble in water, its hydrophobicity. Amino acids that have $U$ at the second position of the codon are hydrophobic, whereas those that have $\mathrm{A}$ at the second position are hydrophilic. Codons for the same amino acid typically vary only at the third position. The third letter is where redundancy lies with eight amino acids with a fourfold degeneracy, where all four bases are interchangeable. In all cases, $\mathrm{U}$ and $\mathrm{C}$ are interchangeable in the third position. In other words, the third position of the codon is information-free with much flexibility. Many amino acids are specified by more than one codon. Codons for the same amino acid tend to have same nucleotides at the first and second positions, but a different nucleotide in the third position. The relative lack of criticality is related to the fact 
that the pairing between anticodons and codons often enjoy a certain flexibility, so those same anticodons can pair with more than one codon, a phenomenon known as wobble [98]. This is why the number of tRNAs and, therefore, of anticodons is smaller than the number of 64 codons, usually ranging between 35 to 45 [57]. Once the code was born, the need to minimize errors might have refined it. The code has been optimized over the eons and isn't simply the product of chance, but of natural selection.

A key role of the universal genetic code is to maintain integrity and verify the specificity of each mRNA codon to a particular amino acid. There must be an accuracy strategy of cross checking that could reveal that mRNA codons and amino acids will interact directly. Various authors have suggested that the original amino acid-binding motifs could have been the actual codons rather than anticodons [96]. But contrawise, we believe the codon-amino acid pairing system might have evolved for code verification at a later stage of code evolution. Initially, anticodons developed between the interactions of pre-tRNA and pre-aaRS [90]. The anticodons selected the codons of pre-mRNA by base-pairing (Fig. 7). As the genetic code was refined and optimized, verification on the strings of codon on the mRNA strand began, through quality control, to ensure that each tRNA successfully interprets the amino acid information for protein synthesis with a low error rate. Most likely the amino acid-codon interaction, mediated by aptamers, evolved later for keeping the code error free.

\section{Coevolution of Translation Machines and the Genetic Code}

The contemporary genetic code of protein biosynthesis most likely evolved from a simpler code and process. Crick [35] suggested that the present code is a random accident 
forever frozen in time, while others argued that the code, like all other features of organisms, was shaped by natural selection. Both the stereochemical and the coevolutionary hypotheses provide possible mechanisms for the selection of amino acids by RNAs from a large pool of prebiotic soup, which are recruited for protein synthesis. During these processes of selection and recruitment of amino acids, the translation machine and the genetic code evolved. Natural selection has led to codon assignments of the genetic code that minimizes the effects of translation errors and mutations during the evolution of the code. The adaptive hypothesis posits that the genetic code continued to evolve after its initial creation, so that the current code maximizes some of the functions.

We accept all three well-known hypotheses - the stereochemical, the coevolutionary, and the adaptive - for the origin and evolution of the genetic code at different stages. We concur with previous researchers that multi-generations of amino acids were produced sequentially $[69,93$, 95] as the code expanded, along with the pathways of amino acid biosynthesis. The biosynthetic relationships between different generations of amino acids are closely linked to the evolution of the genetic code. We contend that information evolved along with the translation machines, and played a vital role in perfecting the translation process and genetic code. Woese [46] suggested that the genetic code and the translation mechanism evolved together in the prebiotic world. Here we elaborate this concept of information-based coevolution of the translation machine and the genetic code that may provide a new window into the origins of translation and the genetic code. A new model of the evolution of genetic code is proposed here: Information-based Coevolution of Translation Machinery and the Genetic Code (ICTC).

The translation machines are an extremely complicated hierarchy of complex macromolecules that are symbiotically related to one another. Yet the whole functions with 
remarkable precision. Once the translation machinery complex for protein synthesis is installed step-by-step, information enters into the system via symbiotic interactions of mRNA, tRNA, aaRS, and ribosome. This machinery implements the genetic code. We summarize here how such a complex translation machinery would evolve step-by-step into today's protein-synthesizing machinery, starting from the cosmic building blocks in hydrothermal crater-lake environment (Fig. 10).

The origin of biomolecular machinery likely centered around the tRNA-amino acid alliance, both being ancient molecules in the hydrothermal vent environment. Various 'spare parts' of biomolecules for building translation machinery were available in the prebiotic soup during the chemical stage from which some few were selected, based upon the chemical affinity between macromolecules. tRNA is the oldest and most central nucleic acid molecule. Its coevolutionary interactions with aaRSs define the specificities of the genetic code. The biochemical pathway outlined here for the emergence of the genetic code is the simplest and the most straight forward account of the development of RNA-dependent protein synthesis.

The information stage emerged from a reciprocal partnership between small ancestral oligopeptides and oligonucleotides. They both contributed initially to rudimentary information coding and catalytic rate accelerations. It begins with the molecular recognition, attraction, and communication between pre-tRNAs and amino acids, mediated by pre-aaRS. The role of tRNA synthetases in the origin of the genetic code is pivotal. It helps the anticodon of tRNA to pair with the right amino acid. It is the matchmaker between tRNA and its corresponding amino acid. Coevolution, the coordinated succession of structural changes mutually induced by the increasingly interacting and growing protein and nucleic acid molecules, played an important role during the origin of translation machinery and genetic code. aaRS coevolved with tRNA and 
tRNA coevolved with mRNA during the rise of the genetic code specificities. Carter and Wills [21] suggested a novel mechanism of how tRNAs are recognized by certain aaRS. Their results indicate that tRNAs carry two codes: the well-known anticodon, and a second one in the acceptor stem (Fig. 6E). These two codes aren't arbitrary: the nucleic acid sequence of the acceptor stem, and the anticodon code for distinct physical properties of amino acids. In other words, the codon/amino acid pairing reflects the different physical roles the different amino acids play in the structure of full, folded proteins. According to them, the genetic coding of 3D protein structures evolved in distinct stages, based initially on the size of the amino acid and later on its compatibility with globular folding in water.

We concur with Woese [46] that the genetic code and the translation mechanism evolved together in the prebiotic world. Here we discuss a simple but effective biological information system that works as a translation system. We show in Fig. 10 the proposed biochemical pathways and coevolution of translation machinery and the genetic code in three stages. We outline how early RNAs and protein catalysts developed into the universal coding system we have today. Our outline is necessarily speculative, but it suggests a series of transitional stages of symbiotic relationships between tRNAs and proteins that may have led to the origin and evolution of the genetic code. Since molecular evolution did not leave any fossil record, some of the transitional stages of the translation machinery are now erased by evolution as the final stage appeared. Thus, no record of code evolution has so far been detected.

$<$ Figure 10 about here $>$

Among different species of RNAs, tRNA has a very ancient history and is more closely associated with protein during synthesis. Pre-tRNA, the tRNA's ancestor likely played a central role of primitive translation early on. A stereochemical relation between some amino acids and 
cognate anticodons of pre-tRNA must have played an important informational role in the earliest assignments.

The origin of the code follows closely the biosynthetic pathways of refining the translation machinery complex in three successive stages in the RNA/protein world (Fig. 11):

- $\quad$ pre-tRNA/ pre-aaRS/pre-mRNA machine.

- tRNA/ aaRS/mRNA machine; and finally,

- tRNA/aaRS/mRNA/ribosome machine.

Each stage represents the mutualism between polynucleotides and proteins. These symbiotic interactions are creative, intimate, and reciprocal exchanges. Polynucleotide and protein are molecules of mutualism, codependent, and complementary that led to the emergence of the genetic code and translation. Evolutionary change in one partner in mutualism triggers change in the other. This is how pre-tRNA changed to tRNA, pre-mRNA to mRNA, and preaaRS to aaRS as the information system is improved. The creation of the ribosome is the culmination of the symbiotic relationships between RNA and protein in the evolution of translation and the genetic code. RNA synthesizes protein in the ribosome and protein synthesizes RNA in polymerase. Cooperative systems facilitated the emergence and evolution of the universal genetic code four billion years ago.

$<$ Figure 11 about here $>$

\subsection{Biological Information Systems}

Biological systems have embedded information structure for supporting their functions [99-102]. An information system can be defined as a set of related components that work 
together for storing, and processing data and for providing information [103]. This definition of an information system views it as an open system. Like an open system, an information system has a purpose and it interacts with its environment. It differentiates and elaborates itself in dealing with the changing environmental conditions just like biological systems. Terms such as "automata" and "machine" refer to a form of an information system.

Even though the ideas of artificial automata came from the observation and study of natural automata such as biological systems, we tend to use man-made machines and other artifacts as a metaphor to better understand the biological systems. Various metaphors such as nanobot, bio-nanobot, etc. have been used in the literature to refer to different types of information system. Metaphors are useful because they are efficient: they transfer a complex meaning in a few words. Some of the popular metaphors including robot and nanomachine are deeply entrenched in our social life, news, and literature. Figure 13 relates these terms to the biological information system. Biological systems exhibit characteristics that relate to processing and using messages that convey information.

We use metaphors such as nanobots and computers for information systems in cells. This practice may be fine as long as we understand the limitations of these metaphors. It's obvious that a cell is more complex than a computer system. The metaphors and analogies explain only a portion of the activities of the biological systems. We believe that, in most cases, a basic-level metaphor is more useful and discriminatory in explaining difficult concepts by association than a higher-level or system-level metaphor. For example, to say 'a cell is a nanobot' is not very revealing about the complexity of a cell. However, it is more meaningful if we say that a cell is a combination of assembler, transcriptor, translator, adapter, pattern-recognizer, pattern-copier, builder, inventory of materials, etc. Metaphors like assembler, translator, adapter, etc. can be 
called as basic-level metaphors. Taken together, they reveal closely the functions and structure of a cell. Another example, a ribosome can be described metaphorically as an assembler that assembles a protein with the help of charged tRNAs. This points out the fact that a ribosome is part of a cell. It also illustrates the hierarchical nature of relationships between biological systems and between metaphors. To our knowledge, there is no higher-level metaphor that adequately describes a cell or, for that matter, any other biological system. A basic-level metaphor or a combination of basic-level metaphors can better describe a biological system. It is important to note that any of these basic-level metaphors can be viewed (modeled) as an information system.

All macromolecules, organelles and cells, no matter how rudimentary, use information and material to conduct their tasks. Information used by them is in various forms such as attractiveness, proximity, pattern, match, symmetry, sequence, rule, and feedback, etc. These informational terms have the usual meanings. Attractiveness between modules relate to various chemical bonds that form easily between them. Proximity refers to the closeness between molecules. A pattern is a configuration of things in a certain way. Match involves similarity and complementarity between molecular elements and surfaces. Symmetry relates to the shape of molecules and organisms. A sequence is a specific order in which related things follow each other. A biological sequence is a molecule that includes smaller molecules such as nucleotides in RNA or amino acids in proteins. Rules specify conditional information. Feedbacks are information in the form of signals. By the time of DNA-mRNA-Protein synthesis, cells had developed a very advanced, stable, and streamlined biological information system to help carry out the translation. Our discussion here is limited to the emergence of the information system in the RNA/protein world, before the appearance of DNA and the first cells. 
The characteristics of biological systems were identified and recognized by early pioneers such as Turing [104,105], Shannon [106], Von Neumann [28], Wiener [107], Bertalanffy [108], and others. They have made tremendous contributions to our understanding of the biological processes by envisioning and proposing the concepts, frameworks, and models that help imitate the biological processes. Turing [104,105] was instrumental in recognizing organized shapes, patterns, forms, and decision making in biological organisms. Shannon [106] formalized the concepts of message, the transmission of message, and the semantic aspect of communication and information. Von Neumann [28] proposed the idea of natural and artificial automata, and developed detailed models to emulate the behavior and actions of natural automata. Wiener [107] enunciated the concepts of control and feedback in systems. Bertlanffy's general systems theory [108] suggests that all systems share some common organizing principles. All these pioneering works in the form of theory, framework, and model have given rise to many advances in technology and biological knowledge. These advances have allowed us to develop better methods to design information systems for the simulation and visualization of biological information systems.

\subsection{Evolution of Biological Information System}

Life may be defined operationally as an information processing system —a structural hierarchy of various functional units - that has acquired through evolution, the ability to store and process the information necessary for its own accurate reproduction. Here, it is very useful to take a wider meaning of the word 'information' as opposed to just the classical definition of information based upon the information theory of Claude Shannon [106]. There are various 
definitions of the word information over the years. Historically, the word information has represented three different types of meanings [109]:

(1) information as the process of being informed,

(2) information as a state of an object, and

(3) information as the disposition to inform.

Information as a process includes the ideas of message communication, meaning, and error due to a noisy channel [110]; information as a state of an object covers the idea of knowledge; and information as the disposition to inform includes the ideas of a capacity of an object to inform another object [114], and information as a specific thing [111].

Information can be signals, natural patterns (including shape, space, size, etc.), match, proximity, attractiveness (i.e., hydrophobicity and hydrophilicity), symmetry, sequence, rules, feedback, instructions, algorithms, content, and as knowledge, etc. [112,113]. A biological information involves all of the above types of information. The biological information also evolved along with the translational machinery and the genetic code. The evolution of biological information can be broadly categorized as GNC (basic), SNS (intermediate) and Universal Genetic Code (advanced) levels of information. A GNC level of biological information has more of a physical nature and includes things like attractiveness, proximity, and pattern. An SNS level of biological information includes match, symmetry, sequence, and signal, in addition to the information at the basic level. A Universal Genetic Code level of biological information adds rules, instruction, feedback, and algorithm to its repertoire (Fig. 13). As implied above, these levels of biological information are cumulative. In other words, an advanced level of biological information also includes both the basic and the intermediate levels of biological information. As protocells evolved their patterns (structures), by way of environmental necessities, the structure 
changed to handle specialized functions. Their structural components differentiated and elaborated to handle specific roles and functions. Protocells started to have a more modular structure where each module played a specialized role(s). Several authors have found evidence of modular structures in organelles and cells $[114,115]$. A module is composed of one or many types of molecules. A modular structure requires a noise-free communication among its modules, in addition to the communication within a module. This scenario uses more information than a simple non-modular structure. The information system used by a protocell has to coevolve to handle a greater information demand as the modular structure of the protocell becomes more and more elaborate and specialized.

The biological information systems became increasingly sophisticated in order to process more and more advanced levels of biological information. Figure 13 shows the proposed coevolution of the biological information systems in three stages. The GNC biological information system dealt mainly with physical, structural, and spatial type of information whereas the UGC biological information system was a sophisticated system capable of handling rules, feedback and instructions, etc. in order to support the various functions of a translation process.

$<$ Figure 13 about here $>$

It is instructive to view information systems at three levels - basic (GNC), intermediate (SNS), and advanced (UGC) level as shown in Figure 13. The basic biological information system can be compared with early man-made information systems such as the Turing machine, and the computer systems of early 1950s. The intermediate biological information system can be compared to a system having more elaborate parts such as memory, data storage, processor, and logic. The computer systems of the 60 s and 70 's can be used as an illustration of the intermediate biological information systems. The advanced biological information system is very modular, 
distributed, and has a sophisticated memory structure and communication mechanism seen today in our man-made information systems. The pre-tRNA/pre-aaRS/pre-mRNA stage used an basic information system to process basic types of information. The tRNA/ aaRS/mRNA stage used an intermediate information system to process intermediate levels information. The aaRS/tRNA/mRNA/ribosome stage used a more advanced information system that was able to process advanced types of information.

All signal processing devices, both analog and digital, have traits that make them susceptible to noise. Noise reduction is a goal to all communication systems. Biological information system is no exception. Biological processes, such as protein synthesis undergo random fluctuations - 'noise' or errors that are often detrimental to reliable information transfer. With the evolution of the code, denoising methods were implemented through the redundancy of codons. A practical consequence of redundancy is that errors in the third positions of the of the triplet codon caused only silent mutations or an error that would not affect the protein because the hydrophilicity or hydrophobicity was maintained by the equivalent substitution of amino acids [57]. The biological information system model is the process of translating the genetic code into corresponding amino acids as an error-prone information channel [110]. In this scenario, evolution drives the emergence of a genetic code as amino acids map that minimizes the impact error. The codon to amino acid assignment is treated as a noisy information channel, when the mapping of codons to amino acids becomes nonrandom. The inherent noise (i.e., error in translation) in the channel poses a problem: how can a genetic code be constructed to withstand noises while accurately and efficiently translating information? The answer is redundancy: several codons can specify a single amino acid. This redundancy implies either that there is more than one tRNA for many of the amino acids or that some tRNA molecules can base-pair with 
more than one codon. In fact, both situations occur. Redundancy explains why so many alternative codons for an amino acid differ only in their third nucleotide (Table 2). De Duve [66] pointed out that in an RNA genome, genes and messenger are one and the same molecule, usually present in numbers of copies. In such a system, innumerable mutations may take place without lethal effects. If one gene molecule and its translation product are disabled, many other unharmed molecules remain to carry on the function involved. Redundancy has been increased considerably from GNC code to SNS code and has become extreme in the universal code for optimization, perhaps to minimize noise or translation errors. We show the plausible correlation between stepwise modifications in the translation machinery and the evolution of the genetic code.

\subsection{Pre-tRNA/pre-aaRS/pre-mRNA Translation Machine}

Our starting point is a protocell which is packed with amino acids and pre-tRNA molecules (Fig. 11A). The molecular attraction between a pre-tRNA and a specific amino acid occurs inside the protocell by molecular selection and stereochemical relation. A specific amino acid begins to join with its cognate pre-tRNA with the help of an assignment enzyme, such as pre-aaRS. This enzyme catalyzes the activation of a specific amino acid. This pre-aaRS has two binding sites: one for a pre-tRNA, the other for an amino acid situated in the vicinity of a catalytic site in such a way that one amino acid is attached to pre-tRNA. The pre-tRNA, then, would hold an amino acid in the first site and recognize it in the second. The correct ligation of the amino acid with a cognate pre-tRNA depends on the specificity of two binding sites. Once 
the amino acid bond is established, four charged pre-tRNA molecules are available to create custom-made pre-mRNA.

Here we show a piecemeal buildup of pre-mRNA by pre-tRNA. A charged pre-tRNA has created its custom-made pre-mRNA strand as a separate storage device by base pairing between anticodon and codon, so the information of the specific amino acid in the anticodon can be transferred to the corresponding codon (Fig. 7D). A symbiotic relationship is established among three components: pre-tRNA, pre-aaRS, and pre-mRNA to create a short chain of amino acids, which form the biosynthetic protein. The protein chain grew through the addition of further residues of amino acids in the same manner. The result was a synthesis of the first coded protein, through the linking of the amino acids that were carried by the pre-tRNAs. At this stage of the GNC code the translation machine began to form (Table 1, Fig. 11). There are four codons in the GNC code that are assigned to four amino acids: valine, alanine, aspartic acid and glycine, from which the first simple protein chain was created (Figs. 11A, Fig. 12). These earliest proteins were the easiest to biologically synthesize. The primitive GNC code was prone to frequent translation errors because of the adverse effect of point mutations, when pre-tRNA molecules began to read the message of pre-mRNA strand.

\section{4. tRNA/aaRS/mRNA Translation Machine}

In the next stage of translation, pre-tRNA evolved into tRNA through gene duplication. Pre-mRNA evolved into mRNA by linking several strands of pre-mRNA to increase the storage capacity. Pre-aaRS became aaRS through ligation to specific tRNA. These three modifications gave rise to the SNS code (Fig. 11B). The superior information bearing qualities of mRNA, the 
superior catalytic potential of aaRS, and better adaptor capacities of tRNA emerged from such complexes with gradual expansion of the genetic code. At this stage, tRNAs selected and recruited six more amino acids (glutamic acid, leucine, proline, histidine, glutamine and arginine) in addition to the GADV amino acids (Fig. 12). These charged tRNAs then create 12 additional codons through base pairing and linking pre-mRNA strands, so that the newly synthesized mRNA strands were more information-rich for storage. mRNAs now possessed at least $16(4+12)$ codons, or combinations of these codons. The mechanism of creating new strands of pre-mRNA are similar as shown in Figure 7. Now two sets of pre-RNA molecules are joined to form a new generation of mRNA. At this stage, the mRNA strands became longer, containing the digital information of 16 codons representing 10 amino acids, or combination thereof allowing for redundancy. The expanded SNS code was refined through the symbiotic interactions of the tRNA/mRNA/aaRS complex. The translation system was considerably improved from the GNC to the SNS stage, but the code remains only moderately robust, susceptible to errors because of the limitation of redundancy. The primitive GNC code expanded to an SNS code, composed of 16 codons (GGC, GGG, GCC, GCG, GAC, GAG, GUC, GUG, CUC, GUG, CCC, CGC, CAC, CAG, CGC and CGG) and 10 amino acids (glycine, alanine, aspartic acid, valine, glutamic acid, leucine, proline, histidine, glutamine and arginine) [56, 69]. The first 10 amino acids, found in the prebiotic environment, have been identified in carbonaceous chondrites. The SNS genetic code is an imprint of the biosynthetic relationships between amino acids (Table 1). As the code expanded, aaRS began to evolve from the earlier pre-aaRS enzyme, and displaced their less efficient precursors. Primordial class I and class II syntheses evolved from ancestral pre-aaRS. At this point encoded proteins are longer, and possess enough amino acid diversity to take on some of the general features of contemporary 
proteins. The mRNA template provides the specifications for the amino acid sequences of the protein gene products. The recipe for the biogenic protein synthesis was inscribed in the codon sequences of mRNA (Fig. 11B).

$<$ Figure 12 about here $>$

\section{5. tRNA/aaRS/mRNA/ribosome Translation Machine}

The final component of the translation machine, ribosome, is enormous, a hybrid of rRNAs and r-proteins. With the participation of the ribosome, the translation machinery became elaborate with tRNA/aaRS/ mRNA/ribosome complexes; this addition enabled higher specificity in the genetic coding. The ribosome was created through the symbiosis of rRNA and r-protein, which increased the efficiency of translation, leading to the universal genetic code with its 20 amino acids and 64 codons. (Fig. 11C) (Table 1). At this stage, tRNAs selected 10 additional amino acids (isoleucine, methionine, threonine, asparagine, lysine, serine, phenylalanine, tyrosine, cysteine, and tryptophan) (Fig. 12). A variety of charged tRNAs then created the corresponding codons through base pairing, forming longer strands of mRNAs. Each mRNA at this stage has the potential of accommodating 64 codons, or any combination thereof. The expanded universal code was stabilized with the symbiotic interactions of the tRNA/mRNA/aaRS complex. Once the ribosome appears in the scene, the translation is considerably refined to facilitate protein synthesis more efficiently. The key chemical step of protein synthesis on ribosomes is peptidyl transfer, in which the growing nascent peptide is transferred from one tRNA molecule to the amino acid bound to another tRNA. Amino acids are incorporated into the growing protein on the ribosome according to the sequence of the codons 
of the mRNA. When a ribosome finishes reading an mRNA molecule, the two subunits split apart. The structure of the universal code is highly robust against mutational and translational errors because of its large allowance of redundancy. Although many deviations from the universal code exist, they are limited in scope and obviously secondary, and would be introduced later in the evolutionary process. Viruses, bacteria, fungi, plants, animals, primates, and humans all use the same code.

\section{Design of Translation Machines and the Genetic Code: A Model-View-Controller Architecture}

Life is characterized and sustained by a number of information rich biological processes that govern cellular functions, and greatly contribute to its overall complexity. A biological process may involve the use of one or more modules within a cell. This involves communication of different types of information such as signals and connectivity etc. between and within a module. Because of this, any study of the origin of life must address the origin of biological information as well. Although information is fundamental to life today, its origin remains largely speculative and neglected. It is important to keep in mind that life and the information system did not necessarily arise simultaneously. In fact, it seems far more likely that information evolved prior to the onset of life, as cellular life is completely dependent of the flow of information from DNA to RNA to protein. Most likely, information systems evolved from the RNA/protein world prior to the emergence of DNA and the first cells (Fig. 1).

\subsection{The Logic of the Genetic Translation Machines}


Living systems are different from the inanimate world. What enters the system is not the same as what exits. A living system is an open system. Living systems gobble up low-energy entropy, degrade the energy, and expel high-entropy energy into the environment [43]. In our model of a translation machine, life's molecular machine complex is encapsulated by a lipid membrane in a vent environment; its network of information processing components is organized by a feedback loop, to extract order out of chaos. The information-directed synthesis of proteins is the central molecular process that makes the diversity of organisms possible. The biological information is more akin to meaning (synthesis of protein) than to entropy.

The choreography of the translation machines led to the genetic code. We cannot allow molecular machines to move random cargos (information) to random places within the protocells. Specific cargos need to go to specific places at specific times. The same is true for every activity of the translation machine. Looking at the precise activities of molecular machines, we realize that evolution is the only way these machines could have come to exist and function precisely in the right place at the right time. Information guides the coordinated activities of the various translation machineries. Evolution is tinkering - the gradual improvements and better adaptations of translation machines in the vent environment. Various enzymes including both the ribozymes and the protein enzymes play crucial roles in translation. By changing the sequence of amino acids and using the trial and error of evolution, myriads of protein enzymes evolved, each custom-made to catalyze a particular reaction.

Translation machinery needs a supply of free energy. In the hydrothermal vent environment, ATP, the molecular currency of energy for intracellular energy transfer was available [29]. Moreover, the vents provided sulfur, iron, manganese — and other nurturing substances. Living systems are different from machines. In our model of a translation machine, 
life's molecular machine complex is encapsulated by a lipid membrane in a vent environment, and is organized in a network of information processing components by a feedback loop to extract order out of chaos.

Modern cells are amazingly crowded, typically with $25-35 \%$ of the space filled by large molecules such as proteins and nucleic acids, which played crucial roles in the origin of the translation system and the genetic code. Translation is more complicated than transcription, because there isn't a one-to-one correspondence of the nucleotides of mRNA and amino acids in the protein. In the process of developing, storing, and processing biological information, tRNAs created several nanomachines such as mRNAs and ribosomes. The sequences of nucleotides in an mRNA strand is read and used to link amino acids in the proper order to form a new protein chain. Translation requires the concerted effort of over 50 different molecular machines: some made of protein enzyme, some made of RNA, and some made of a combination of both the protein enzyme and RNA [116]. Molecular machines are very specific for the jobs that they do. Nucleic acids are the main information-carrying molecules in the cell, and, by directing the process of protein synthesis, they determine the inherited characteristic of every living thing. As an information system, their main function is to create, encode and store information. A typical enzyme, on the other hand, accelerates chemical reaction, and selects useful biomolecules for creating the information system. It sifts through myriads of biomolecules and grabs the one that it needs. Biological molecules perform this amazing task through molecular recognition.

\subsection{Simulation of Translation Machines and Cells}


The interest of computer scientists regarding the question of origin of life dates back to the origins of computer science. Several attempts have been made to simulate the functions of a molecular translation machine. The field of bio-inspired digital software/hardware was pioneered by Von Neumann [28]. His self-reproducing automata is now regarded as one of the greatest theoretical achievements in the early stages of artificial life research. He found striking parallel between artificial automata (such as a computer) and natural automata such as various nanobots in the cells. He introduced the concept of Universal Constructor (UC), a self-constructing machine, which is capable of building any other machine, provided it can access its description or information tape. This approach was maintained in the design of his cellular automata, which is much more than a self-replicating machine. The UC is more like a Turing machine with a tape control that could store and execute instructions. There are three components of von Neumann's UC machine:

- a memory tape, containing the description (a one-dimensional string of elements;

- the constructor itself, a machine capable of reading the memory tape and interpreting its contents; and

- a constructing arm, directed by the constructor used to build the offspring (the machine described in the memory tape).

A universal constructor with its own description could build a machine like itself. To complete the task, the universal constructor needs to copy its description and insert the copy into an offspring machine. Von Neumann noted that if the copying machine made errors, these mutations would provide inheritable changes in the property, like the evolutionary process. He realized that the biological machine is much more sophisticated than his UC. Unlike mindless automata, which must be told exactly what to do in order to build the correct objects, a biological 
machine plays a dual role: it contains instructions - an algorithm - to make a certain kind of translation machine and related enzymes (e.g., mRNA, tRNA, ribosome, aaRS and other enzymes), but additionally it can be blindly copied as a merely physical structure without reference to the instructions. Another major difference between UC and evolving natural organisms is the lack of feedback in the fitness channel of the former. Cellular automata have been useful artificial models for exploring how relatively simple rules, combined with spatial memory, can give rise to complex emergent patterns; it may be relevant for understanding new questions about the cell division and its relation to information. Subsequently, von Neumann's UC has been modified by several workers to create Artificial Life. However, von Neumann's UC has the information system outside the machine. So, when UC reproduces its offspring, it lacks the information tape. It has to be added each time during reproduction. It is analogous to vesicle division - a mechanical division of an empty protocell, devoid of instruction.

\subsection{Reading the Message in $m R N A$}

Once an mRNA has been produced by tRNA, the information present in its nucleotide sequence is used to synthesize a protein. Each molecule of mRNA encodes the information for one protein. The conversion of the information of mRNA into proteins represents a translation of information into another language that uses considerably different symbols. We begin with the reading of the message encoded in mRNA by a network of three molecules - tRNA, aaRS, and ribosome - the core of the translation machines - to produce protein. The genetic data needed to assemble a protein is stored in the mRNA strand using the four-letter alphabet $\mathrm{U}, \mathrm{C}, \mathrm{A}$, and $\mathrm{G}$ in triplet bases or codons. On the other hand, proteins are made of twenty different kinds of amino 
acids. The codons in the mRNA molecule do not recognize the amino acids they specify: the codons do not, for example, bind directly with the amino acid; the protein and RNA languages seem unrelated. How, then, is the message of mRNA read? We know that tRNA serves as a reading device through base-pairing. The actual step of translation from mRNA into the protein language occurs when amino acids and tRNAs are matched and joined. As in all translations, there must be someone, or something, that is bilingual. Recognition is entirely done in RNA language through the help of the bilingual translator aaRS. This enzyme can recognize both an amino acid and its corresponding tRNA, because both contributed to the birth of aaRS, as discussed in 4.4 section. Finally, the ribosome, a perfect mobile assembler (nanobot), links amino acids together in the order specified in mRNA to make proteins. In decoding the genetic message from mRNA, using a communication metaphor, mRNA plays the role of a channel, which communicates the genetic message to the ribosomes, which use charged tRNAs as the decoder. The genetic message is decoded by the ribosomes from the sixty-four codons of mRNA to the twenty-letter alphabet of the protein. The ribosomal RNAs in ribosomes are programmed to recognize the codon as it appears on the mRNA. In the RNA/protein world, the information flow is simple: mRNA to protein.

But an mRNA strand with strings of codons is a relatively small and simple molecule with limited storage capacity. It can store small amounts of genetic information; the capability of a ribozyme as an enzyme is severely limited. However, this catalytic deficiency is compensated by a variety of enzymes that are available in the hydrothermal vent environment. The short life of mRNA makes the protocell very responsive to changing conditions in the environment. Later, more durable DNA would emerge to become the molecule of choice for the large storage of genetic information for protein synthesis, replacing mRNA from its main function. The new 
generation of mRNA is created by transcription of DNA. mRNA becomes a daughter of DNA to carry out its specific instruction of translation and protein synthesis.

However, our discussion is centered around the RNA/protein world. There was no DNA at that time. It's amazing how these abiotic pioneer polymers such RNA and proteins communicated symbiotically and created the translation system and the genetic code step by step that has been working with exquisite precision for the last four billion years. How did such a complicated and specific system as the translation and the genetic code arise in the first place, and from an utterly random and vast array of building blocks in the primordial vent environment? We don't know. This is the greatest enigma in molecular biology [81]. Here we speculate a plausible scenario for the origin of the translation system, and the genetic code in the RNA/protein world.

There is no doubt that the advent of the coded proteins utterly changed the conditions of emerging life. A fundamental property of coded protein synthesis is that amino acids are not added in haphazard fashion. Their sequence is rigorously imposed by mRNA. In the vent environment noncoded proteins were synthesized by linking available amino acids in a random fashion. Some of these enzymes were useful for their specificity with substrates, others were discarded for biosynthesis that lacked specificity. As we discussed earlier, as more and more specific enzymes were needed for biosynthesis, many of the noncoded proteins became superfluous for complex chemical reactions; the demand for specific, coded enzymes would have been in greater need than noncoded enzymes, so that the range of specificity in the catalytic activity could be increased. But the synthesis of coded proteins was more complex than the noncoded proteins, and it required a repertoire of complex machinery parts. Perhaps it all started with certain RNA molecules reacting with certain amino acids, otherwise it is difficult to see 
how else RNA molecules could have become involved with the linking together of amino acids $[66]$.

The crucial step was the chemical bonding of particular amino acids to small pre-tRNA molecules with specific anticodon sequences to communicate between two different languages. As more and more pre-tRNA molecules began to attract specific amino acids, mediated by preaaRS, the charged pre-tRNA attracted nucleobases in their anticodons by base pairing. These nucleobases were joined in triplet forming pre-mRNA molecule, which functioned as a separate device to store amino acid assignments of the pre-tRNA molecule. Eventually, pre-tRNA molecules began to translate its custom-made pre-mRNA strands, creating rudimentary coded polypeptide. Our suggestion is that the initial pre-tRNA/amino acid attraction happened not as part of a protein-synthesizing apparatus, but to improve the range and efficiency of chemical reactions in the RNA/protein world. The evolution of coded protein synthesis may have been driven by the accumulation of incremental advances of the translation machines which led initially from pre-tRNA/amino acid interaction to eventual production of complex protein machine consisting of tRNA/mRNA/aaRS/ribosome machine. The transition from noncoded to coded proteins was the stimulus for the emergence of the translation and the genetic code; they were developed step by step through the process of natural selection.

\subsection{Genetic Code Vs. Binary Code}

Genetic code is often compared with the binary code used by the computers. Significant similarities and differences exist between the two types of the code. Each system has its advantages and limitations. The primary or source alphabet used in computers and electronic 
communication is the binary digit $(0,1)$, or a bit, a contraction for 'binary digit.' [106]. The bit is the smallest unit of information on a computer. Binary information is grouped into sets of eight bits, called bytes; each byte thus has one of $2^{8}$ or 256 possible configurations of zeroes and ones. A byte is just 8 bits and is the smallest unit of memory that can be addressed in many computer systems.

Shannon [106] understood that a binary source alphabet could be extended by forming ordered pairs, ordered triplets, ordered quadruplets, and so forth to form receiving alphabets larger than two. In molecular biology, these extensions are called codons. The simplest unit of mRNA, on the other hand, is the nucleotide, which can have one of four bases-A, U, C, and G, the quaternary 'bit' [117]. However, we think that the use of bit in quaternary system of mRNA is a misnomer. Here we choose a new name in the genetic code, called qit, or quaternary digit instead of bit. This increased variation means that each nucleotide of mRNA can hold twice as much information as each digit of a binary program. The qit creates more algorithmic randomness than bit and is more information rich. Shannon's great insight in information theory is entropy. Entropy measures the degree of uncertainty or randomness in a system. Entropy is the opposite of information. It destroys information. We can reduce the entropy to the point where stored information becomes maximal and transmission is highly reliable. Genetic information is low in entropy and high in information content. Errors in translation from nucleotides to amino acids are like noises or entropy. This is why the genetic codes is evolved in three stages to minimize the errors or entropy incrementally during translation.

In mRNA, the genetic information comes in triplets of nucleotides or codons, which represent different amino acids, meaning that each codon in mRNA has only $4^{3}$ or 64 possibilities. Each codon is thus an extension, or 'byte' and has exactly as much information as a 
6-bit byte, or in computer terminology a code word, since $2^{6}$ is 64 possible sequences for codons. Here we use a new terminology for representing genetic information, called 'qyte' instead of 'byte.' In our terminology, each qyte is 3 -qit long, giving $4^{3}$ possibilities.

Both binary and genetic codes contain signals that indicate where to begin and end the reading of their messages. Computers use start and stop bits for this purpose, while the genetic code contains one start codon and three stop codons. In a binary code, a single inaccurate bit causes its byte to have a different value, which can cause significant errors. However, mRNA exhibits greater flexibility and is more resilient in comparison, as many nucleotide changes do not result in changes to the value of the amino acids coded by a codon.

However, information contained in life exists in two forms, digital (genetic) and analog (metabolism), and both appeared concurrently in the RNA/protein world [45]. Digital information is encoded in linear polymers such as DNA and RNA in discrete codons, analog information is manifest in the differing concentrations of biomolecules especially proteins that get passed from generation to generation. Analog information systems dominate in the first three hierarchical stages, namely cosmic, geological, and chemical, but in the last two stages, namely information and biological, digital information systems dominate. Recognizing that there are two sequential events, first the origin of an analog chemical system capable of adaptive evolution and then a digital revolution, the origin of life problem becomes much more tractable [118]. Acceptance of this dichotomy and this progression, help resolve the question of dual roles of RNA and proteins in generating information systems.

\subsection{Conversion of Three Letter Codons into Numerical Codons}


The genetic code is obviously not the result of a random assignment of codons to amino acids. It has a structure; synonyms are grouped. The language-based terminology of the genetic code reflects the fact that both genes and proteins are essentially 1-dimensional arrays of chemical letters. The nucleic acid alphabet comprises of four chemical letters, A, U, C, and G, whereas proteins are built from twenty different amino acids, represented by 20 abbreviated letters. In order to better visualize the codon distribution in the universal genetic code table, we substitute nucleobase alphabets of mRNA with numbers as follows: 1 for $\mathrm{U}, 2$ for $\mathrm{C}, 3$ for A, and 4 for G. [In case of DNA codons, 1 represents thymine (T).] We have now created a universal numerical codon matrix in a structural format consisting of 64 numerical codons that specify 20 amino acids, and the start and stop codons (Table 2).

$<$ Table 2 about here $>$

In Table 3, the abbreviation of the universal genetic code table is shown in numerical codons with redundancy. Each matrix cell displays information in numerical codon and its corresponding amino acid. Because of numerical distribution of codons in rows and columns, one can visualize the distribution of codons and their redundancy easily in the matrix cells; it was less obvious in standard genetic code using combinations of four letters. Looking at Table 3, we can say that codons beginning with 4 formed first, followed by codons with 2 . Codons with prefix 1 and 3 were added last at the genetic code table.

$<$ Table 3 about here $>$

In Table 4, we have shown a 1-letter abbreviation of 20 amino acids, and its corresponding numerical codons. We have used 3 additional letters, J, X, and Z (shown in bold font) to signify 3 stop codons namely opal, ochre, and amber respectively.

$<$ Table 4 about here $>$ 
Using these 3 tables as guides, we have developed a software to simulate the translation of the numerical codon sequence of mRNAs to produce its corresponding amino acid sequence. We name this software as 'Codon-Amino Acid-Translator-Imitator' or (CATI) that mimics the process of reading a sequence of codons and translating it into a sequence of the corresponding amino acids and vice versa. The CATI software can handle the reverse process also where a sequence of amino acids is translated into a sequence of corresponding codons. Table 5 shows some sample outputs of CATI. Table 5 is made up of several sections. In the first section, column one shows a given set of numerical codon sequences. Column 2 shows the corresponding amino acid sequences. CATI accepts inputs two ways - from an excel spreadsheet and from a set of randomly generated sequences. A user can create a set of numerical codon sequences using a spreadsheet. CATI can also generate a random sequence of numerical codons of an arbitrary length for translation.

Table 5 shows some sample outputs of CATI. Table 5 is made up of several sections. In the first section, column one shows a given set of numerical codon sequences (read from a spreadsheet). Column 2 shows the corresponding amino acid sequences. Table 5 shows the translation of randomly generated numerical codons also. The second section of table 5 shows the translation of randomly generated numerical codons. Table 5 also shows the output of the reverse process - translating a given sequence of amino acids into the corresponding sequence of numerical codons. The third, fourth, and fifth sections of table 5 show the translation of given amino sequences into the corresponding numerical codon sequences. Since a given aminosequence can form from several possible codon sequences, we show the count of all possible codon sequences and just a few actual codon sequences in the table. The last section in table 5 shows the conversion of DNA codon sequences into the corresponding numerical codon 
sequences. Using the distribution of numerical codons, we can visualize at least some of the steps by which nature might have invented the code.

$<$ Table 5 about here $>$

There is a great potential of application of numerical codons in bioinformatics. For example, it can be used in translating codon sequences in DNA sequencing in numerical forms, which is the process of determining the precise order of nucleotide bases within a DNA molecule. The advent of rapid DNA sequencing methods has greatly accelerated biological and medical research and discovery. The use of numbers instead of letters may expedite similarity searches between two strands of DNA. Thus, numerical codons can be used for DNA barcoding of a species, or DNA profiling of a person as a parallel system. The Genome Sequence Data Base (GSBD), operated by the National Center for Genome Resources (NCGR), is a national database of publicly available nucleotide sequences and associated biological and bibliographic annotation. In a pilot study, the data of a small gene can be converted to a numerical codon sequences by our CATI software, for a feasibility study to see whether it affords better DNA mining, alignment of two DNA sequences, and for searching methods, storage, and data retrieval systems in the future.

The CATI software is based on the Model View Controller (MVC) design pattern [25, 119]. The MVC facilitates the design idea by segregating functional/task responsibilities and assigning them to different components/module of software. This leads to an architecture with components that are relatively independent of each other. The three major components are (Fig. $15 \mathrm{~A})$ :

- The Model is the central component of the pattern. It manages the data (information), its associated logic, and rules of application. 
- The View is a (visual) representation of the model, the user interface. It relates to the logic (code) that produces the output. A view can be a form of any output representation of information.

- The Controller accepts input and acts a monitor to mediate (i.e., coordinate) between the tasks of view and model. It handles events generated by the user and communicates those changes to the Model, which updates its state accordingly and communicates any changes back to the Controller. The Controller then updates the view to reflect those changes.

$<$ Figure 15 about here $>$

These three architectural components of the system enable us to imitate the biological information process in a flexible and modular way. It is important to point out that the MVC design pattern is very similar to the implementation architecture of John von Neumann's Universal Constructor of the self-reproducing automata. Given a description, the Universal Constructor produces theoretically any automata from available parts. The universal constructor has not yet been manufactured physically. However, several attempts have been made to implement the universal constructor computationally. Pesavento [120] describes a very good implementation of John von Neumann's self-reproducing machine. The overall architecture of this implementation is shown in figure 15B. It has four components- the State Control Area, the Reading Loop Area, the Memory Area, and the Writing Loop Area. The State Control Area is the overall coordinator of all the other components. The Reading Loop Area reads the tape information and stores them temporarily in the memory area. The information in the memory area is used by the Writing Loop Area to produce the output. We suggest that there is a very close similarity between the Universal Constructor's architecture as shown in figure 15B and the 
MVC Design Pattern. The Controller of the MVC design pattern corresponds to the State Control Area, the Model corresponds to the combination of the Reading Loop Area and the Memory Area, and the View corresponds to the Wring Loop Area. In fact, the MVC design pattern can be interpreted as a more modern operationalization of John von Neumann's Universal Constructor.

We have found that the MVC design pattern is very good at abstracting the natural protein translation machine into an architecture that is modular, and neatly separates the various aspects of information processing into three roles - model, view, and controller.

\subsection{Algorithmic Design of CATI}

We now present the algorithm used by CATI. For simplicity, the algorithm shows the overall logic without dividing it into the MVC components shown in Figure 16. CATI uses a codon chart in the form of a codon-amino mapping shown in Table 5. CATI is given a sequence of numerical codons. CATI can generate a random sequence numerical codon on its own also. This sequence of numerical codons is then translated into the corresponding sequence of amino acids.

$<$ Figure 16 about here $>$

Figure 17 shows the algorithm. The main step of the algorithm in Fig. 17 is the third step of CATI. It uses the ideas of a pattern recognizer (step 3.a), an adapter (step 3.b), and a sequence builder (step 3.c). CATI, in essence, plays the combined role of ribosome, aaRS, and tRNA taken together.

$<$ Figure 17 about here $>$

\section{Simulation and Visualization of the Translation Pathways}


Computer simulations are useful in evolutionary biology for hypothesis testing, for verifying analytical methods, for analyzing interactions among evolutionary processes, and are widely used in different disciplines. In general, computer simulations allow the study of complex systems, including those analytically intractable [121]. Here we use forward simulation models of primitive machines to advance translation machines, mimicking the biosynthetic processes for the origin of the genetic code, and for testing our hypothesis of the coevolution of the translation machines and the genetic code.

In this section we visualize the early stages of translation machine evolution in three stages. We use a simulation and modeling software called AnyLogic, which is commercially available (www.anylogic.com), to simulate and visualize the translation machines. We simulate translation machines at three levels of evolution:

- pre-tRNA/pre-aaRS/pre-mRNA

- $\quad$ tRNA/aaRS/mRNA

- tRNA/aaRS/mRNA/ribosome

Although the molecular organization of the genetic code is now known in detail, how the code came into being has not been satisfactorily addressed. We have already discussed the coevolution of translation machines and the genetic code in chapter 6 ; this offers a simple relation between the codon reading efficiency and the accuracy of the codon translation machine. Here we highlight some of the features of this coevolution for visualization. The rapid and accurate translation of genetic code into proteins is the hall mark of the information stage; it evolved in three distinct stages through the availability of amino acids, and the improvement of the translation machine. It is now widely accepted that the earliest genetic code did not encode all 20 amino acids found in the universal genetic code, as some amino acids have complex 
biochemical pathways and were probably not available in the prebiotic environment. Therefore, the genetic code evolved as pathways for synthesis of new amino acids became available $[69,85$, 95]. In our view, the code evolved in step with the amino acid biochemistry, and the refinement of the translation machine (Fig. 12).

\subsection{Stage I. Visualization—pre-aaRS-pre-tRNA-pre-mRNA Machinery}

The first information system emerged in the prebiotic world as primordial version of the translation machine and the genetic code. The most primitive translation machine consists of preaaRS/pre-tRNA/Pre-mRNA molecules. Four primitive GADV amino acids specific to four pretRNAs, and four pre-aaRS enzymes began to translate the genetic information from pre-mRNA, and to synthesize short polymer chains of protein (Fig. 18). The code that evolved at this stage was the primitive GNC code [69] involving 4 codons (GGC, GCC, GAC, and GUC), which created 4 GADV amino acids (glycine, alanine, aspartic acid, and valine). Since there was no redundancy at this stage, the translation errors are high.

$<$ Figure 18 about here $>$

The informational associations among these biomolecules are shown in the form of an information structure. This information structure showing macromolecules and their association with each other can be captured in the form of a class diagram (Fig. 19). In a class diagram, a rectangular shape represents an informational object. The solid lines connecting these objects show an associative relationship between the objects. It is important to note that only a few attributes of each object are shown in the class diagrams. This is for illustration purposes only. Here, our focus is mainly on the interaction among the objects and not on providing a comprehensive list of attributes for each object. Figure 19 shows the information structure during 
the first stage of the genetic code, the GNC code. pre-tRNA, pre-AARS, pre-mRNA, amino acid, codon, anticodon, as well as protein, and nucleotide are shown as objects in this figure. The relationships shown in a class diagram are static, structural, and associative. A class diagram does not show any dynamic or temporal relationship.

$<$ Figure 19 about here $>$

A pre-AARS attaches the appropriate amino acid to its pre-RNA with the correct anticodon. A pre-mRNA has a sequence of codons. These are generally a short-length sequences dealing with the GNC genetic codes. An amino acid carrying pre-RNA was able to base pair with codons in a pre-mRNA, and helped produce a protein as per the information in the pre-mRNA. At this stage, the types of information used are generally in the form of attractiveness, proximity, and pattern. The right combination of a catalyst, information, and the material acts as a translation machine to produce a new biological artifact. A pre-aaRS/pre-tRNA/pre-mRNA machine's MVC architecture shows a collaboration among these three machine parts that control the formation of a biosynthetic protein chain (Fig. 20). The controller uses pre-mRNA, amino acid, \& anticodon, etc. as information to translate (convert) a codon into the corresponding amino acid with the help of a charged pre-tRNA which acts as an adaptor. The charged pre-tRNA is shown as a view. It produces the amino acid, as an output, based upon its anticodon matching with the codon in premRNA. These amino acids become part of a sequence in the form of a protein chain.

$<$ Figure 20 about here $>$

A visualization of the stage I translation machine has been created using Anylogic software. Appendix A in the supplemental materials provides instructions on how to run the visualization model in the AnyLogic cloud. The visualization model shows the overall translation process of how various molecules interact dynamically to produce a protein. 


\subsection{Stage II. Visualization—aaRS-tRNA-mRNA Machinery}

The translation machine is refined to the second stage (Fig. 21) with the development of aaRS/tRNA/mRNA machine, which increases efficiency and decreases translation errors. At this stage, the GNC code evolved into transitional SNS code with 16 codons (GGC, GGG, GCC, GCG, GAC, GAG, GUC, GUG, CUC, GUG, CCC, CGC, CAC, CAG, CGC and CGG), which code 10 amino acids (glycine, alanine, aspartic acid, valine, glutamic acid, leucine, proline, histidine, glutamine and arginine) $[69,95]$. Because of the redundancy of codons, the translation error is minimized.

$<$ Figure 21 about here $>$

Figure 22 shows the information structure of the aaRS/tRNA/mRNA translation machine during the second stage of the universal genetic code. At this stage, additional information in the form of match, symmetry, and sequence are also available. A tRNA is transformed into a charged tRNA by the aaRS as shown in figure 22 . The anticodon of the charged tRNA matches with the corresponding codon in mRNA. A protein chain is formed by the decoding of mRNA by tRNA.

$<$ Figure 22 about here $>$

Using the MVC framework, we suggest that, in the case of an aaRS machine, proteins represent the 'output', the corresponding charged tRNAs and amino acid ligation (aa-tRNA) as a 'view', and a combination of aminoacyl tRNA and aaRS as a 'controller,' and mRNA as a 'model' that holds codons as information (Fig. 23). The directional arrows represent the control and 'communication' between the various parts of the machine. For example, an aaRS 
coordinates and facilitates the activities of mRNA, tRNA, and amino acid ligation in the formation of protein chain. The aaRS acts as a facilitator and helps produce (select) the amino acid that matches with the codon in mRNA. Briefly, the overall logic of the second stage machine is as follows: specific tRNA binds with a particular amino acid, tRNA, then incorporates the amino acid into a growing protein at a position determined by the anticodon, the anticodon matches with a codon in mRNA, the codon acts as an information carrier that matches with the specific tRNA. The final result is the release of the linked amino acids, which are the protein chain.

$<$ Figure 23 about here $>$

In the supplementary materials section, we show the instructions on how to run the visualization model for the second stage of translation machine.

\subsection{Stage III. Visualization-aaRS-tRNA-mRNA-Ribosome Machine Complex}

By the third stage, the translation machine has fully evolved, now consisting of the aaRS/tRNA/mRNA/ribosome machine that brings forth the universal genetic code (Fig. 24). Translation of the universal genetic code into protein by ribosomes requires precise mRNA decoding by tRNA. At this stage, ribosomes emerged to facilitate a high-fidelity translation. About 31 tRNAs and 20 aaRS enzymes assigned 64 codons specifying 20 amino acids. Of these 64 codons, 61 represent amino acids, and three are start and stop signals. Although each codon is specific to only one amino acid, the code is degenerate, because a single amino acid may be coded for more than one codon. The redundancy of the universal genetic code optimized translation errors and mutations [88]. Codons for the same amino acids tended to bundle together. Perhaps the organization of the amino acids with particular sequences of the code 
minimized the errors that crept into the proteins. Among the 20 amino acids in the universal code, about half came from the prebiotic soup; as we see in the SNS code, the remaining half of amino acids were derivatives of the first set of 10 primitive amino acids by biosynthesis [67].

$<$ Figure 24 about here $>$

Figure 25 shows the information structure available during the third stage of the genetic code. During this stage, a ribosome uses rules, and feedback types of information, in addition to the other types of information during translation. A ribosome acts like a biological assembly machine in the translation of mRNA into protein. A ribosome performs the protein synthesis with the assistance of two other kinds of molecules - mRNA and tRNA.

$<$ Figure 25 about here $>$

Figure 26 shows an MVC model of the ribosome machine. A ribosome machine is sometimes equated with a factory with several machines. It uses other machines such as an aaRS machine to complete the translation process. A ribosome plays the role of the controller. mRNA is a model containing the information in the form of a sequence of codons. An aa-tRNA machine plays the role of a view that supplies an amino acid. Note that this machine is depicted in figure 23. Here, the ribosome machine uses the aa-tRNA machine as it's submachine (sub part), signifying a functional hierarchy among macromolecules. The ribosome produces the peptide chain (protein) by establishing the proper match (fit) between an aa-tRNA and the corresponding codon in the mRNA.

It is well documented that the translation of mRNA into an equivalent protein goes through the process of initiation, elongation, and termination. A ribosome conducts the translation and aaRS provides charged tRNAs continuously throughout the entire translation process. 
In the translation process of the third stage, the translation machine, with the assistance of a ribosome, is visualized using an AnyLogic model. We show the instructions in the supplementary materials section how to run the third stage of the visualization model.

\section{Discussion and Conclusion}

The origin of life as an extraordinary event is the product of two worlds: interstellar space and Earth. The building blocks of life came from interstellar space via meteorites, but first life was synthesized in the womb of our planet. To paraphrase Carl Sagan, life is made of stardustfrom the debris of supernova explosion, but concentrated by Earth. Life is widely believed to have evolved from a simpler, self-replicating molecular system. However, the origin of the structural and informational biopolymers (i.e. proteins and RNAs), and the evolutionary path that led to early life remains obscure. We suggest that hydrothermal crater lakes in the Eoarchean protocontinents were the most plausible cradle for the origin of life.

The key processes in the evolution of the information systems within the biomolecules, emerged simultaneously and naturally from an RNA/protein partnership, thus eliminating the need for a prior RNA world. During the information stage, there was a spontaneous change of prebiotic synthesis within the hydrothermal vent environment, going from disorder to order. Here disorder means a chaotic assemblage of molecules, out of which order emerged when certain preferred biopolymers began to exchange information [33]. As macromolecular machines evolved, they were aided and abetted by their embedded coevolutionary biological information systems to perform more and more complex functions.

We have reviewed the bottom-up pathways of prebiotic synthesis that address several hallmarks in living systems, such as the encapsulation and protocell division, RNA/protein 
world, information processing, energy transduction, and adaptability. The scenarios for the origin of the translation machinery and the genetic code outlined here are both sketchy and speculative but follow those biosynthetic pathways. It's the informational role of RNAs, aided by a series of enzymes, that is key to transforming nonliving chemistry into translation machines and the genetic code.

There are several novel ideas in this symbiotic view of the origin of life that is presented in this paper:

1. We recognize five stages of the hierarchical origin of life: cosmic, geologic, chemical, information, and biological - as a series of ascending levels with increasing complexity of biomolecules, each stage leading to the next stage. In this scenario, life arose around four billion years ago through five hierarchical stages of increasing molecular complexity.

2. During the Late Heavy Bombardment period, asteroids delivered the fundamental building blocks of life, and water to early Earth.

3. Asteroid collisions on young Earth created innumerable hydrothermal crater lakes in the Eoarchean crust. Small hydrothermal crater lakes, through subterranean vent networks, became the perfect cradle for prebiotic chemistry. Various chemicals and energy sources were available in the vent environment. These cosmic compounds concentrated and simmered like a soup in the hydrothermal crater-caldrons. A biosynthetic variety of organic compounds mixed and matched, and in time attained a propitious recipe of rich biomolecules.

4. A new symbiotic model for the origin of life within the hydrothermal crater lakes is proposed. Through symbiosis, these complex biopolymers gradually assembled into 
membrane-bound protocells. At each stage of the evolutionary progression, through natural selection, they refined with increasing stability and complexity, ultimately leading to the first cells.

5. In our view, the RNA/protein world was more parsimonious in the vent environment than the popular RNA world hypothesis. It is easier to make proteins than RNAs in the vent environment. The duality of replication and metabolism is the intrinsic property of life and must have appeared simultaneously before the origin of the first cells. Both RNAs and proteins worked in tandem to jumpstart the life assembly.

6. Endosymbiosis and encapsulation of amino acids, RNAs, proteins, enzymes, and other biomolecules played critical roles in the prebiotic synthesis in the hydrothermal vent environment.

7. The Information stage is a crucial step in the origin of life prior to the origin of DNA and the first cell. We emphasize that reproduction is not possible without information. Life is information stored in a symbiotic genetic language. Information is an emergent property in the RNA/protein world. The molecular attraction between tRNA and amino acid led to the translation machinery and the genetic code.

8. Supply and demand for specific coded enzymes over noncoded enzymes in the RNA/protein world heralded the age of information. The crucial step was the ligation of a specific amino acid to its corresponding pre-tRNA molecule that created a repertoire of complex machinery parts for translation. tRNA is an ancient molecule that created custom-made mRNA for the storage of amino acid assignment. During this stage, translation and the genetic code coevolved. 
9. New views for the piecemeal buildup of translation machines consisting of tRNAs, mRNAs, aaRS, and ribosomes are proposed.

10. We review three main concepts on the origin and evolution of the genetic code and suggest an additional idea, specifically, the coevolution of the translation machines and the genetic code. We suggest that there were three stages in the evolution of the genetic code- GNC, SNS, and finally the universal genetic code. The code evolved through the progressive refinery of translation machines, from pre-tRNA/pre-aaRS machines, to tRNA/aaRS/mRNA machines, and finally tRNA/aaRS/mRNA/ ribosome machines. The evolution of the translation machine reflects the incremental enrichment of information content in the genetic bank of mRNA. The information systems embedded in the translation machines coevolved and became more and more sophisticated as the translation machines became more and more complex.

11. Using a computer simulation, and a visualization model of the possible biosynthetic pathways that led to the origin of the information system, we show the step-by-step evolution of the translation machines and the genetic code.

12. The information age with the origin of translation and the genetic code was a watershed event in the origin of life about four billion years ago.

We have reviewed some of the hierarchical stages that may have accounted for the early states in the emergence of life on Earth. Repeated natural selection and symbiosis at each stage led from billions of highly random and chaotic molecules in the hydrothermal vent into more ordered and organized systems of limited molecules of increasing complexity during prebiotic synthesis. The information age is the most crucial stage in the origin of life, culminating with the emergence of translation and the genetic code, that specific moment when the biotic processes 
become more deterministic. Although biochemical pathways outlined in this paper are reconstructed from multidisciplinary evidence, we believe it should be possible to test the different parts of our model by careful experiments.

Acknowledgements: We thank Mavis Liang for inviting us to contribute this article in the special issue of The Origin of and Early Evolution of Life volume. We owe an immense debt of gratitude to the many authors, who have helped us with their thoughtful, well-documented, and enlightening expositions in the origin of life research. We thank Oliver McRae for reading the manuscript for clarity and brevity. We thank Volkan Sarigul and Oliver McRae for illustrations. This work was supported by P. W. Horn Professor grant from Texas Tech University. 


\section{References}

1. Marchi, S.; Bottke, W.F.; Elkins-Tanton, L.T.; Bierhaus, M.; Wuennemann, K.;

Morbidelli, A.; Kring, D.A. Widespread mixing and burial of Earth's Hadean crust by asteroid impacts. Nature 2014, 511, 578-582.

2. Mojzsis, S.J.; Harrison, T.M.; Pidgeon, T.T. Oxygen-isotope evidence from ancient zircons for liquid water at the Earth's surface 4,300 Myr ago. Nature 2001, 409, 178-181.

3. Chatterjee, S. The RNA/protein world and the endoprebiotic origin of life In Earth, Life, and System; Clarke, B. Ed; Fordham University Press, New York, 2015; pp. 39-79.

4. Chatterjee, S. A symbiotic view of the origin of life at hydrothermal impact crater lakes. Phys. Chem. Chem. Phys, 2016, 18, 20033-20046.

5. Bernstein, M.P.; Sandford, S.A.; Allamonda, L.J. Life's fur flung raw material. Scient. Amer. 1999, 263, 42-49.

6. Chyba, C.; Sagan, C. Endogenous production, exogenous delivery and impact-shock synthesis of organic molecules: an inventory for the origin of life. Nature, 1992, 355:125132.

7. Deamer, D.W.; Dworkin, J.P.; Sandford, S.A.; Bernstein, M.P. Allamandola, L.J. The first cell membranes. Astrobiol. 2002, 2, 371-381.

8. Grieve, R.A.F.; Cintala, M.J.; Therriault, A.M. Large-scale impacts and the evolution of Earth's crust: the early years. In Processes of the Early Earth Reimold, W.U.; Gibson, R.L., Eds.; Geological Society of America Special Paper, Boulder, 2006; 405, pp. 23-31.

9. Mojzsis, S.J.; Harrison, T.M. Vestiges of a beginning: Clues to the emergent biosphere recorded in the oldest known sedimentary rocks. GSA Today, 2000, 10,1-5. 
10. Baross, J.A.; Hoffman, S.E. Submarine hydrothermal vents and associated gradient environment as sites for the origin and evolution of life. Origins of Life, 1985, 15, 327345.

11. Martin, W.; Baross, J.; Kelley, D.; Russell, M.J. Hydrothermal vents and the origin of life. Nature Rev Microbiol, 2008, 6, 805-814.

12. Mulkidjanian, A.Y.; Bychkov, A.B.; Diprova, D.V.; Galperin, M.Y.; Koonin, E.B. Origin of first cells at terrestrial, anoxic geothermal fields. Proc. Nat. Acad. Sci. USA, 2012,109, E821-E830.

13. Mishteyn, D.; Damer, B.; Havid, J; Deamer, D. Amphiphilic compounds assemble into membranous vesicles in hydrothermal hot spring water but not in seawater. Life, 2018, 8, $11 ; 10.3390 /$ life 8020011 .

14. Pearce, B.K.D; Pudriz, R.E.; Semenov, D.A.; Henning, T.K. Origin of the RNA world; The fate of nucleobases in warm little ponds. Proc. Nat. Acad. Sci. USA, 2017,114, 11327-11332.

15. Tang, M.; Chen, K.; Rudnick, R.L. Archean upper crust transition from mafic to felsic marks the onset of plate tectonics. Science, 2016, 351, 373-375.

16. Chatterjee, S. The hydrothermal impact crater lakes: the crucibles of life's origin. In Handbook of Astrobiology; Kolb, V.M. Ed.; CRC Press; Taylor \& Francis: Boca Raton, FL, USA, 2018; pp.265-295.

17. Chatterjee, S. The origin of life in hydrothermal impact crater lakes: A hierarchical model of the prebiotic synthesis. In Earth and Life II, Talent, J. A. Ed.; Springer, Dordrecht, in press (unpublished). 
18. Cockell, C.S. The origin and emergence of life under impact bombardment. Phil. Trans. R. Soc. 2006, B361, 1845-1856.

19. Kring, D.A. Impact events and their effect on the origin, evolution, and distribution of life. GSA Today, 2000, 10(8), 1-7. '

20. Osiniski, G.R.; Tornabene, L.L.; Banerjee, N.R.; Cockell, C.S.; Flemming, R.; Izawa, M.R.M.; McCutcheon, J.; Parnell, J.; Preston, L.J.; Pickersgill, A.E.; Pontefract, A.; Sapers, H.M.; Southam, G.. Impact-generated hydrothermal systems on Earth and Mars. Icarus, 2013, 224, 347-363.

21. Carter, C.W. Jr.;Wills, P. R. Interdependence, reflexivity, impedance matching, and the evolution of genetic coding. Mol. Biol. Evol. 2017, http://dx.doi.org/10.1093/molbev/msx265.

22. Harish, A.; Caetano-Anolles, G. Ribosomal history reveals origin of modern protein synthesis. PLoS One, 2001, doi:e32776.

23. Bowman, J.C.; Hud, N.V.; Williams, J.D. The ribosome challenge to the RNA world. J. Mol. Evol., 2015, 80, 143-161.

24. Hazen, R.M. Genesis: The Scientific Quest for Life. Joseph Henry Press: Washington, D.C., USA, 2005.

25. Küppers, B.O. Information and the Origin of Life. MIT Press, Cambridge, MA, USA, 1990.

26. Walker, S. I.; Davies, P. C. W. The algorithmic origins of life. J. R. Soc. Interface, 2012. doi: http://dx.doi.org/10.1098/rsif.2012.0869.

27. Reenskaug, T. MODELS - VIEWS - CONTROLLERS. Technical note, Xerox PARC, December, 1979, http://heim.ifi.uio.no/ trygver/mvc/index.html. 
28. Von Neumann, J. Theory of Self-Reproducing Automata. University of Illinois Press: Chicago, IL, USA, 1966. Edited and completed by A.W. Burks.

29. Martin, W.F.; Sousa, F. L.; Lane, N. Energy at life's origin. Science, 2014, 344, 10921093.

30. Deamer, D. W. First Life: Discovering the Connections between Stars, Cells, an How Life Began; University of California Press, Berkeley, CA, USA, 2012.

31. Damer, B.; Deamer, D.W. Coupled phases and combinatorial selection in fluctuating hydrothermal pools: a scenario to guide experimental approaches to the origin of cellular life. Life, 2015, 5, 872-887

32. Shapiro, R. A simpler origin of life. Scient. Amer. 2007, 296, 24-31.

33. Dyson, F. Origins of Life. Cambridge University Press: Cambridge, UK, 2004.

34. Wachterhäuser, G. The cradle of chemistry of life: on the origin of natural products in a pyrite-pulled chemoautotrophic origin of life. Pure Appl. Chem., 65, 1993, 1343-1348.

35. Crick, F.H.C. The origin of genetic code. J. Mol. Biol., 38, 1968, 367-379.

36. Cech, T.R. RNA as an enzyme. Scient. Amer., 1986, 255(5), 64-75.

37. Cech, T.R. Crawling out of the RNA world. Cell, 2009, 136, 599-602.

38. Gilbert, W. The RNA world. Nature, 319, 1986, 618.

39. Orgel, L.E. The origin of life. Scient. Amer., 1994, 271(4), 77-83.

40. Robertson, M.P.; Joyce, G.F. The origins of the RNA world. Cold Spring Harb. Perspect Biol., 2012, 4(5), . a003608.

41. Cech, T.R.. The ribosome is a ribozyme. Science, 2000, 280, 878-879.

42. De Duve, C. The beginnings of life on earth. Amer. Scient., 83, 1995, 428-437.

43. Davies, P. The Fifth Miracle. Simon \& Schuster, New York, USA, 1999. 
44. Crick, F.H.C. Central dogma in molecular biology. Nature, 1970, 227, 561-563.

45. Koonin, E.V. Why the central dogma: on the nature of biological exclusion principle. Biology Direct, 10, 2015, 52, DOI: 10.1186/s13062-015-0084-3

46. Woese, C. Evolution of the genetic code. Naturwissen, 1973, 60, 447-459.

47. Osawa, S. Evolution of the Genetic Code; Oxford University Press: Oxford, UK, 1995.

48. Woese, C.R. The Genetic Code: The Molecular Basis for Genetic Expression; Harper \& Row: New York, USA, 1967.

49. Pizzarello, J.R.; Cronin, J.R. Non-racemic amino acids in the Murchison and Murray meteorites. Geochem. Cosmochem. Acta, 64, 2000, 329-338.

50. Bada, J.L. New insights into prebiotic chemistry from Stanley Miller's spark discharge experiments. Chem. Soc. Rev., 2013, 42, 2186-2196h.

51. Maynard Smith, J.; Szathmary, E. The Origins of Life; Oxford University Press: New York, USA, 1999.

52. Chen, I.A. Prebiotic chemistry: replicating towards complexity. Nature Chem. 2015, 7, 101-102.

53. Svoboda, P.; Cara, A. Hairpin RNA: a secondary structure of primary importance. Cell. Mol. Life Sci., 2006, 63, 901-908.

54. Eigen, M.; Winkler-Oswatitsch, R. Transfer-RNA, an early gene. Naturwissen, 1981, 68, 282-292.

55. Woese, C.R. On the evolution of the genetic code. Proc Nat Aca Sci USA, 54, 1965, $1546-1552$

56. Di Giulio, M. The origin of tRNA molecule: implications for the origin of protein synthesis. J. Theor. Biol., 2004, 226, 89-93. 
57. Freeman, S. Biological Science, $2^{\text {nd }}$ edition, Pearson Prentice Hall: Upper Saddle River, New Jersey, USA, 2005.

58. Szathmary, E. The origin of the genetic code: amino acids as cofactors in an RNA world. Trends Genet. 15, 1999, 223-229.

59. Di Giulio, M. On the origin of transfer RNA molecule. J Theor Biol., 1992, 159,199-209.

60. Di Giulio, M. Was it an ancient gene codifying for a hairpin RNA that, by means of direct duplication, gave rise to the primitive tRNA molecule? J. Theor. Biol., 1995, 177, 95-101.

61. Tamura, K. Origins and early evolution of the tRNA molecule. Life, 5, 2015, 1687-1699.

62. Tanaka, T.; Kikuchi, Y. Origin of cloverleaf shape of transfer RNA-the double hairpin model: implication for the role of tRNA intro and long extra loop. Viva Orig. 2001, 29, $134-142$

63. Widmann, J.; Di Giulio, M.; Yarus, M.; Knight, R. tRNA creation by hairpin duplication. J. Morph. Evol. 2005, 61, 524-530.

64. Nagaswamy, U.; Fox, G.F. RNA ligation and the origin of tRNA. Orig. Life. Evol. Biosph. 2003, 33, 199-209.

65. Lahav, N. Prebiotic co-evolution of self-replication and translation in RNA world? $J$. Theor. Biol., 2001, 151, 531-539.

66. De Duve, C. Singularities: Landmarks on the Pathways of Life: Cambridge University Press, New York, USA, 2005.

67. Wong, J.T.F. A co-evolution theory of the genetic code. Proc. Nat. Acad. Sci. USA, 1975, 72, 1909-1912. 
68. Wong, J.T.F. Coevolution of genetic code and amino acid biosynthesis. Trends Biochem. Sci. 1981, 6, 33-36.

69. Ikehara, K. Origins of gene, genetic code, protein and life: comprehensive view of life systems from a GNC-SNS primitive code hypothesis. J. Biosc., 2002, 27, 165-186.

70. Noller, H.F. The driving force for molecular evolution of translation. $R N A, \mathbf{2 0 0 4}, 10$, $1833-1837$.

71. Noller, H.F. Evolution of protein synthesis from an RNA world. Cold Spring Harb. Special Biol., 2012, 2012, 4:a003681.

72. Ramakrishnan, V.. The ribosome: some hard facts about its structure and hot air about its evolution. Cold Spring Harb. Symp. Quant. Biol., 2010, 74, 155-179.

73. Altstein, A.D. The progene hypothesis: the nucleoprotein world and how life began. Biol. Dir., 2015, 10, 67 doi: 10.1186/s13062-015-0096-z.

74. Ban, N.; Nissen, P.; Hansen, J.; Moore, P.B.; Steitz, T.A. The complete atomic structure of the large ribosomal unit at 2.4 A resolution. Science, 2000, 289, 905-920.

75. Noller, H.F. On the origin of ribosome: coevolution of subdomains of tRNA and rRNA. In The RNA World: Cold Spring Harbor Laboratory Press, Plainview, NY, USA, pp. 137$156,1993$.

76. Schimmel, P.; Alexander, R.W. All you need is RNA. Science, 1998, 281, 658-659.

77. Nissen, P.; Hansen, J.; Ban, N.; Moore, P.B.; Steitz, T. A. The structural basis of ribosome activity in peptide bond synthesis. Science, 2000, 289, 920-929.

78. Orelle, C.; Carlson, E.D.; Szal, T.; Florin, T.; Jewett, M.C.; Mankin. A.S. Protein synthesis by ribosomes with tethered subunits. Nature, 2015, 524, 119-124. 
79. Savir, Y.; Tlusty, T. The ribosome as an optimal decoder: a lesson in molecular recognition. Cell, 153, 2013, 471-479.

80. Alberts, B.; Johnson, A.; Lewis, J.; Raff, M.; Roberts, K.; Walter, P. Molecular Biology of the Cell, $4^{\text {th }}$ edition. Garland Science, New York, USA, 2002.

81. Koonin EV; Novozhilov, A.S. Origin and evolution of the genetic code: the universal enigma. lubmb Life 61, 2008, 99-111.

82. Woese, C.R. On the evolution of cells. Proc. Nat. Acad. Sci. USA, 2002, 99, 8742-8747.

83. Dunhill, P. Triplet nucleotide-amino acid pairing: a stereochemical division between protein and non-protein amino acids. Nature, 1966, 210, 25-26.

84. Yarus, M.; Widmann J.J.; Knight, R. RNA-amino acid binding: a stereochemical era for the genetic code. J. Mol. Evol., 2009, 69, 406-429.

85. Wong, J.T.F. Emergence of life: from functional RNA selection to natural selection and beyond. Front. Biosci. 2014, 19, 1117-1150.

86. Freeland, S.J.; Knight, R.D.; Landweber, L.F.; Hurst, L.D.. Early fixation of an optimal genetic code. Mol. Biol. Evol. 2000, 17, 511-518.

87. Szathmary, E.. The origin of the genetic code: amino acids as cofactors in an RNA world. Trends Genet. 1999, 15, 223-229.

88. Rodin, A.S.; Szathmary, E.; Rodin, S.N. On the origin of genetic code and tRNA before translation. Biol. Dir. 2011, 6, 14, doi: http://www.biology-direct.com/content/6/1/14.

89. Ling, J.; Reynolds, N.; Ibba, M. Aminoacyl-tRNA synthesis and translation quality control. Ann. Rev. Microbiol., 2009, 63, 61-78.

90. Giege,' R.; Sissler, M.; Florentz, C. Universal rules and idiosyncratic features in tRNA identity. Nucleic Acid Res., 1998, 16, 5017-5035. 
91. Poole, A.M.; Jeffares, D.C.; Penny, D. The path from the RNA worlds. J. Mol. Evol. 1998, $46,1-17$.

92. Yarus M. RNA-ligand chemistry: a testable source of genetic code. $R N A \quad \mathbf{2 0 0 0}, 6,475-$ 484.

93. Wong, J.T.F.; Ng, S.K.; Mat, W.K.; Hu, T.; Xue, H. Coevolution theory of the genetic code at age forty: pathway to translation and synthetic life. Life, 2016, 6, 12, doi:10.3390/life6010012

94. Eigen, M.; Schuster, P. The hypercycle: a principle of natural self-organization. Part a: emergence of the hypercycle. Naturwissens, 64, 1997, 541-565.

95. Di Giulio, M.. An extension of the coevolution theory of the origin of the genetic code. Biol. Dir., 2008, 3, 37 doi.org/10.1186/1745-6150-3-37.

96. Knight, R.D.; Landweber, L.F. Rhyme or reason: RNA-arginine interactions and the genetic code. Chem. Biol., 1998, 5, R215-220.

97. Copley, S.D.; Smith, E.; Morowitz, H.J. A mechanism for the association of amino acids with their codons and the origin of genetic code. Proc. Nat. Acad. Sci. USA, 2005, 102, $4442-4447$.

98. Crick, F.H.C. Codon-anticodon pairing: the wobble hypothesis. J. Mol. Biol. 1966, 19, $548-555$.

99. Kampfner, R. R. Biological information processing: the use of information for the support of function. Biosystems, 1989, 22, 223-230.

100. Moreno, A.; Ruiz-Mirazo, K. 2011. The Informational nature of biological causality. In Information and Living Systems: Philosophical and Scientific Perspectives, Terzis, G., Arp, G.R.Eds.; MIT Press: Cambridge, MA, USA, pp.157-175. 
101. Shanks, N.; Pyles, R. A. Problem solving in the life cycles of multicellular organisms: Immunology and Cancer. In Information and Living Systems: Philosophical and Scientific Perspectives, Terzis, G., Arp, G. R., Eds; MIT Press: Cambridge, MA, USA, 2011; pp.157-175.

102. Miller, W. B. Biological information systems: Evolution as cognition-based information management. Prog. Biophys. Mol. Biol., 2018, 134, 1-26.

103. Zwass, V. Information System, 2016, https://www.britannica.com/topic/informationsystem.

104. Turing, A. M. On computable numbers, with an application to the Entscheidungsproblem: A correction. Proc. Lond. Math. Soc., 1937, 43 (2), 544-546.

105. Turing, A.M. The chemical basis of morphogenesis. Phil. Trans. R. Soc. Lond., B 237, $1952,37-72$.

106. Shanon, C. E. A mathematical theory of communication. Bell Sys. Tech. J., 1948, 27, $379-423 \& 623-656$.

107. Wiener, Norbert (1965). Cybernetics, Second Edition: or the Control and Communication in the Animal and the Machine, The MIT Press, 1965.

108. Bertalanffy, L. von. General System Theory: Foundations, Development, Applications. George Braziller Publisher: New York, USA, 1968.

109. Adriaans, P. Information. In The Stanford Encyclopedia of Philosophy (Fall 2018 Edition), E. N. Zalta, Ed.; doi: https://plato.stanford.edu/archives/fall2018/entries/information.

110. Tlusty. T. A. A model for the emergence of the genetic code as a transition in the noisy information channel. J. Theor. Biol. 2007, 292: 331-342. 
111. Buckland, M. Information as a thing. J. Amer. Soc. Inform. Sci. 1991, 42, 351-360.

112. Floridi, L. The logic of being informed. L. Anal. 2006, 49, 433-460.

113. Price, J. R. An Introduction to Information Theory: Symbols, Signals, and Noise. Dover, New York, 1980.

114. Hartwell, L. H.; Hopfield, J. J.; Leibler, S.; Murray, A. W. From molecular to modular cell biology. Nature, 1991, 402, C47-C52.

115. Dagley, M. J.; Lithgow, T. 12 - TOM and SAM machineries in mitochondrial import and outer membrane biogenesis. In The Enzymes, R. E. Dalbey; C. M. Koehler, F. Tumanoi, Eds., 25, 309-343. Academic Press: New York.

116. Goodsell, D. S. The Machinery of Life. Springer: New York, US A, 2010.

117. Yockey, H. P. Information Theory, Evolution, and the Origin of Life. Cambridge University Press: Cambridge, UK, 2005.

118. Baum, D. A.; Lehman, N. Life's late digital revolution and why it matters for the study of the origins of life. Life, 7, 2017, 34, doi: 10.3390/life7030034.

119. Reenskaug, T. The Model-View-Controller (MVC) Its Past and Present. 2003, http:/heim.ifi.uio.no/ trygver/2003/javazone-jaoo/MVC_pattern.pdf.

120. Pesavento, U. An Implementation of von Neumann's Self-Reproducing Machine. Artificial Life, 1995, 2, 337-354.

121. Arenas, M. Computer programs and methodologies for the simulation of DNA sequence data with recombination. Front. Gen. 2013, 4, 9, doi: 10.3389/fgene.2013.00009. 


\section{Figure captions}

Figure 1. The hierarchical origin of life, viewed as four ascending stages of increasing complexity showing biomolecules in the prebiotic world that led to the development of the first cells. These stages are: cosmic, geologic, chemical, information, and finally biological. Each higher level provides novel emergent properties not found in any of the lower level. These sequential steps took place in the dark, hot environments of the hydrothermal crater-lake basins, which may well represent the Earth's oldest ecosystem.

Figure 2. The crucible of life. During the Early Archean period ( $\sim \mathrm{Ga})$, freshwater crater basins with hydrothermal vent system at their central peak, would have offered a protective sanctuary for the origin of life. The boiling water was rich with organic molecules brought by meteorites. On the water's surface, primitive lipid membranes and hydrocarbons float like an oil slick. The minerals along the floor of the basin acted as a catalytic surface for the concentration and polymerization of monomers. The bubbling, biotic soup was thoroughly mixed by convection currents. These same currents also circulated some of the lipid membranes down to the basin floor where they attached to the porous mineral layers, encapsulating biopolymers such as RNA and amino acids. Heat, gases, and chemical energy such as ATP released from the hydrothermal vent brewed and condensed the prebiotic soup, which began to collect at the mineral substrate, at the bottom of the basin (after Chatterjee, 2016).

Figure 3. Hydrothermal crater lakes become the cradle for the origin of life. The highly-cratered surface of Earth's first supercontinent Vaalbara is pockmarked with hydrothermal crater lakes for biosynthesis. Moonrise on the Vaalbara supercontinent (after Chatterjee, 2018).

Figure 4. Six main steps represent the early evolution of non-coding RNA in the hydrothermal crater vent environment. In the first stage, there is an assortment of different nucleotides (including some not found in RNA). In the second stage, these nucleotides randomly assemble into polynucleotides by polymerization with the removal of water molecules. In the third stage, four nucleotides, A, U, G, and C were selected out during replication by the Watson-Crick base pairing. In the fourth stage, nucleotides undergo polymerization to create a mixture of polynucleotides that are random in length and sequence. In the fifth stage, a variety of 
biomolecules from the vent environment such as amino acids, mononucleotides, oligonucleotides, and peptides are randomly encapsulated, creating molecular crowding. Because of crowding, the single-stranded RNA begins to fold, forming the double-stranded stem and single stranded loop that make the hairpin. In the sixth stage, this secondary structure of RNA is shown separately: it forms a ribozyme and begins to act as an enzyme. Stems are created by hydrogen bonding between complementary base pairs. The ribozyme acquires amino acids, at the CCA sequence of the stem, as "cofactors" increasing its catalytic efficiency. The opposite end of the loop consists of 3 , unpaired bases facing outward, forming a binding site for the attaching of three corresponding mononucleotides. This is the beginning of the emergence of the proto-tRNA.

Figure 5. The origin of hairpin ribozyme and its chemical bonding with appropriate amino acid. A single-stranded RNA can develop secondary structure by infolding with double-stranded stem and single-stranded loop forming a hairpin ribozyme. The ribozyme acquired amino acid as cofactor to form a more efficient catalyst [51]. The amino acid is bound to an oligonucleotide (RNA molecule containing only 3 nucleotides) by an activation enzyme such as pre-aaRS (preaaRS 1 in figure), and the oligonucleotide is bound to the surface of the ribozyme by base pairing (ribozyme 1). The activating enzyme 2 would bind the next batch of amino acid and oligonucleotide is attached to ribozyme 2 , forming the peptide bond.

Figure 6. The double-hairpin model of the tRNA formation, showing its evolutionary transitions (after Giulio 1992, 2004; Tanaka and Kikuchi 2001). A-B, shows a secondary hairpin structure of two RNA molecules (such as ribozymes), each with a stem and a loop: the CCA sequence at the $3^{\prime}$ end of the stem offers a binding site for an amino acid, whereas the 5 ' end offers a binding site for phosphorous; $\mathrm{C}$, the direct duplication or ligation of the hairpin structure may have generated a double hairpin structure, creating a D-hairpin and a T-hairpin. An anticodon (ANT) site forms between the two stems. In this newly configured pre-tRNA molecule, the acceptor site and anticodon site are now closer together, enabling it to decode a pre-mRNA molecule for protein synthesis (see Fig. 20); D, a schematic diagram showing the salient features of the pretRNA molecule, with the anticodon site; E the contemporary full-length tRNA molecule could have been formed by the ligation of two half-sized pre-tRNA structures. Its acceptor stem bases and anticodon stem/loop bases, at the tRNA 5 '-half and the 3 '-half, fit the double-hairpin 
folding. This suggests that the primordial double-hairpin RNA molecules could have evolved to modern tRNA. This new secondary structure of tRNA resembles a cloverleaf, its anticodon end forms a complementary base pair with the mRNA codon; F, a cloverleaf from nature illustrates the structural similarity with the new tRNA molecule; $G$ a schematic diagram showing the salient features of the tRNA molecule, emphasizing the anticodon. The tRNA serves a crucial role in matching an amino acid with a specific codon. When tRNA is bound to an amino acid it is called an aminoacyl tRNA. There is now a corresponding tRNA, with an appropriate anticodon, for each amino acid.; $\mathrm{H}$, the cloverleaf secondary structure of tRNA then folds to the L-shaped tertiary structure. At the CCA minihelix end, the aminoacylation site interacts with a large ribosomal unit for a peptide bond formation. The opposite end interacts with the small ribosomal subunit, to decode mRNA triplets through codon-anticodon interactions.

Figure 7. Primitive translation process began with interaction between pre-mRNA and pre-tRNA before the appearance of ribosomes. Pre-tRNA molecule serves as a crucial role in matching a prebiotic amino acid to a specific codon. A, a pre-tRNA molecule with two hairpin loops of $3^{\prime}$ and $5^{\prime}$ terminals and an anticodon (ANT); the acceptor stem at the $3^{\prime}$ end forms a covalent attachment to a specific amino acid that corresponds to the anticodon sequence; $\mathrm{B}$, schematic representation of pre-tRNA emphasizing the 3' end and corresponding anticodon; $\mathrm{C}$, encapsulated pre-tRNA and pre-mRNA molecule with codon-anticodon interaction; the inner cell membrane acts as a substrate to hold the pre-mRNA molecule in place. $\mathrm{D}$, the anticodon of a pre-tRNA molecule began to hybridize with corresponding nucleotide by base pairing; the triplet nucleotides were kinked to form a codon; In the abiotic stage, the primitive GNC code appeared, which code four amino acids: valine, alanine, aspartic acid, and glycine (Di Giulio 2008); E, codons thus produced by pre-tRNAs began to link in a strand to form a pre-mRNA with coding sequence; F, pre-tRNA and pre-mRNA interactions to form rudimentary translation; the 3' acceptor end of pre-tRNA gathers appropriate amino acid from the pool and binds it by activation enzyme; an aminoacetyl pre-tRNA with appropriate anticodon hybridizes with codon, ejecting the pre-tRNA; the next aminoacyl pre-tRNA then moves down another codon and repeats the process; amino acid released from the old pre-tRNA begins to join to form a protein chain. 
Figure 8. The origin of the ribosome. The ribosome consists of two subunits each with specific roles in protein synthesis. The basic form of the ribosome has been conserved in evolution. Perhaps, the early ribosome was similar to that of modern prokaryotes, which is a large ribonucleoprotein complex of 3 rRNAs and 52 r-protein molecules. Although ribosomal proteins greatly outnumber ribosomal RNAs, the rRNAs pervade both subunits. There is now evidence that rRNA interacts with mRNA or tRNA at each stage of translation, and that ribosomal proteins are necessary to maintain rRNA in a structure in which it can perform the catalytic functions. Most likely, the symbiotic interactions of ribosomal RNAs and ribosomal proteins gave rise to ribosomes, which grew by accretion. There is some controversy however whether the small or large subunit appeared first. In our view, both units coevolved by accretion of ribosomal RNAs and ribosomal proteins.

Figure 9. The translation machinery of the ribosome, where the mRNA message is decoded. The ribosome provides the substrate for controlling the interaction between mRNA and aminoacyltRNA. A, an aminoacyl tRNA, with appropriate anticodon. B, each ribosome has a binding site for mRNA, and three binding sites for tRNA. The tRNA binding sites are designated E-, P-, and A-sites (for exit, peptidyl-tRNA, aminoacyl-tRNA, respectively). The small subunit contains the binding site for mRNA. Translation takes place in a four-step cycle (C-F) that is repeated over and over during the synthesis of protein. C, in step 1, an aminoacyl-tRNA, with appropriate anticodon, enters the vacant A-site on the ribosome where it hybridizes with a codon. D, in step 2, the carboxyl end of the protein chain is uncoupled from the tRNA at the P-site, then joined by a peptide bond to the free amino group of the amino acid linked to the tRNA at the A-site. This reaction is catalyzed by an enzymatic site in the large subunit, called peptidyl transferase (PT). E, in step 3, a shift in the large subunit (shown by arrow) relative to the small subunit in the 3' direction, moves the two tRNAs into the E- and P-sites of the large unit, and ejects the empty tRNA from E-site. F, in step 4, the small subunit moves exactly three nucleotides along the mRNA molecule, bringing it back to its original position relative to the large subunit. This movement resets the ribosome with an empty A-site so that the next aminoacyl-tRNA molecule can bind. The cycle repeats when the incoming aminoacyl-tRNA binds to the codon of the A-site (modified from Panno 2010). G, summarizes the life cycle of the ribosome during its translation. 
Figure 10. The inferred biochemical pathways for the origin of translation and the genetic code in the RNA/peptide world. The hydrothermal crater vent was crowded with several monomers such as amino acids and nucleotides, which were polymerized on the mineral substrate to form various proteins and RNAs. As ribozymes evolved into pre-tRNAs, each pre-tRNA molecule captures specific amino acid, assisted by pre-aaRS enzyme. Eventually, anticodons of pre-tRNAs created custom-made pre-mRNAs for storage of genetic information. The interaction between pre-tRNA and pre-mRNA generated small protein chain by rudimentary translation and GNC primitive code with four codon and four amino acids. With the refinement of translation, pretRNA evolved in tRNA and pre-mRNA to mRNA with the expansion SNS code with 16 codons, and 10 amino acids. Finally, as ribosome appeared by fusion of ribosomal proteins and RNA, it facilitates high-fidelity translation, leading to universal genetic code with 64 codons and 20 amino acids.

Figure 11. The inferred temporal order of evolution of translation machinery systems showing coevolution of translation machines and the genetic code. In our model, there are three stages of translation machinery systems: (1) pre-tRNA/pre-aaRS/pre-mRNA stage when GNC code evolved with the beginning of translation system; (2) tRNA/aaRS/mRNA stage when SNS code appeared; and finally, (3) tRNA/aaRS/mRNA/ ribosome stage when universal code evolved.

Figure 12. Three stages of the evolution of the genetic code corresponding to the evolution of the translation machines and the progressive addition of amino acids. Pre-tRNA molecule creates its custom-made pre-mRNA for storage of limited amino acid information in the beginning. Primitive translation process began with interaction between pre-mRNA and pre-tRNA. PretRNA molecule in collaboration with pre-aaRS enzyme serve as crucial role in selecting and matching prebiotic amino acids from the prebiotic soup. At this stage, translation machine is simple consisting of pre-tRNA/pre-aaRS/pre-mRNA. In the abiotic stage, the primitive GNC code appeared, which code four amino acids: valine, alanine, aspartic acid, and glycine (Di Giulio 2008) [95]. In the next stage, translation machine becomes modified and efficient with the evolution of the tRNA/aaRS/mRNA translation machine, when six new amino acids-glutamic acid, leucine, proline, histidine were created. mRNA strand becomes more elongated and containing 16 codons and combination thereof with assignments of 10 amino acids with the 
emergence of the SNS code [95]. These 10 amino acids were readily available from the prebiotic environment [93]. Here we see the beginning of degeneracy, where some the amino acids have more than one codon assignment. With the appearance of ribosome, the SNS code is modified to universal genetic code with 64 codons and 20 amino acids. The translation machine containing tRNA/aaRS/mRNA/ribosome becomes more robust with extensive degeneracy minimizing translation errors and mutation. Furthermore, amino acids with similar chemical properties seem to share similar codons. Ten more new amino acids were recruited at this stage from SNS stage: isoleucine, methionine, threonine, asparagine, lysine, serine, tryptophan, phenylalanine, tyrosine, and cysteine, totaling 20 amino acids. These new amino acids are derivatives of the first set of 10 primitive amino acids [93]. mRNA becomes independent storage device, and can create its own strand by replication without the assistance of tRNA. mRNA strand becomes more elongated, containing information of 20 amino acids using 64 codons or combination thereof.

Figure 13. Evolution of Biological Information Systems. The basic biological system during the inception of the GNC code mainly processes the stereochemical properties of tRNA anticodons and GADV amino acids. The intermediate biological system during the origin of SNS code is able to process matching signals and signals etc. The advanced biological system during the origin of the universal genetic code is able to process rules, feedback, and instructions.

Figure 14. A hierarchy of Information Systems and Nanobots. This diagram shows a unified definition of various terms used for molecular systems. It related the idea of an information system with terms like 'nanomachines,' etc.

Figure 15. A, a Model-View-Controller (MVC) Architecture pattern, and B, an implementation Architecture of von Neumann's Universal Constructor (UC). The solid arrows in the figure show the flow of control among components. For example, the solid arrow between the controller and model implies that the controller directs the actions of the model. A dotted arrow indicates a flow of data (information).

Figure 16. An overall architecture of CATI based on the MVC pattern. It shows the controller, model, and view aspects of the logic. 
Figure 17. The overall Logic (Algorithm) of CATI. The logic combines the role of ribosomes, aaRS, tRNA, and mRNA (?) into a single overall process.

Figure 18. The pre-aaRS/pre-tRNA/pre-mRNA translation machine. Pre-aaRS is the matchmaker between pre-tRNA and amino acid. Four primitive amino acids and their cognate four pre-tRNAs and pre-aaRS molecules were selected from the prebiotic soup. Each amino acid with its specific pre-tRNA molecules were catalyzed by pre-aaRS enzyme in presence of ATP to create a charged pre-tRNA molecule. In a similar way, four charged molecules were available to decode the short string mRNA one at a time. During hybridization of anticodon of pre-tRNA with codon of pre-mRNA, each pre-tRNA delivers the appropriate amino acid, which is linked to form a chain of biosynthetic protein for the first time, containing four amino acids. This is the first stage of translation, when primitive GNC code evolves.

Figure 19. A class diagram showing an information structure during the first stage of translation system. This diagram shows relationships among various parts of the primitive translation machine. Pre-tRNA attaches to a specific amino acid with help of pre-aaRS molecules. The charged pre-tRNA molecule has an anticodon that hybridizes with the corresponding codon of pre-mRNA. As pre-tRNA begins to decode pre-mRNA molecules, short chain of protein is synthesized for the first time in prebiotic environment. The linkage of an amino acid to a pretRNA established the primitive GNC genetic code.

Figure 20. An MVC architecture of a pre-aaRS/pre-tRNA/pre-mRNA machine. Pre-aaRS and pre-tRNA direct charged pre-tRNA that will decode pre-mRNA to a growing protein.

Figure 21. The aaRS/tRNA/mRNA translation machine. Ten primitive amino acids joined with specific tRNA molecules by aaRS enzymes to form a pool of 10 charged tRNA molecules. These charged tRNA molecules begin to decode mRNA, creating a chain of longer, biosynthesized protein molecule. At this stage, SNC code appears with 10 amino acids for 16 codons. The translation is moderately efficient with the appearance of redundancy to minimize the translation errors. 
Figure 22. A class diagram showing the interactions of aaRS/tRNA/mRNA translation machine showing the information structure during the origin of the SNS code. At this stage, 10 primitive amino acids are available to create 10 or more charged tRNAs for decoding mRNA. An amino acid in the charged tRNA will be incorporated into a growing protein chain, at a position dictated by the anticodon of the tRNA.

Figure 23. An MVC architecture of aaRS/tRNA/mRNA translation machine. aaRS and tRNA facilitate the interaction between a charged tRNA and a mRNA. A charged tRNA is an adaptor that acts as a view and help release the amino acid to form a chain of protein.

Figure 24. The aaRS/tRNA/mRNA/ribosome translation machine. tRNA delivers amino acid to ribosome that serves as the site of protein synthesis. Each ribosome has a large 50S subunit and a small 30S subunit that join together at the beginning of decoding of mRNA to synthesize a protein chain from amino acids carried by a tRNA. The correct tRNA enters the A site of the ribosome and appropriate amino acid is incorporated into the growing peptide chain, which transfers from tRNA in the P site to the tRNA of A site. As ribosome moves, both tRNAs, and mRNA, then shits to the E site. Each newly translated amino acid is then added to a growing protein chain until ribosome completes the protein synthesis. At this stage, universal genetic code is optimized with 20 amino acids for 64 codons including start and stop codons. The translation is highly efficient with start and stop codons; redundancy minimizes the translation errors and mutations.

Figure 25. Class diagram showing the interactions of aaRS/tRNA/mRNA/ribosome translation machine. The diagram is similar to that of figure 22. In addition, it shows the introduction of a ribosome which decodes mRNA with the help of charged tRNA.

Figure 26. An MVC structure of aaRS/tRNA/mRNA/ribosome translation machine. A ribosome is a part of a bigger machine which uses aaRS machine to decode the mRNA information into a corresponding sequence of amino acids to link a protein chain. 
Table 1. The evolution of the Universal Genetic Code is seen in three distinct stages. The nucleotide sequence of an mRNA (in a digital format) is translated into the amino acid sequence of a protein (in an analog format) via the genetic code. Genetic information is encoded in mRNA using codons, comprised of four bases: uracil (U), cytosine (C), adenine (A), and guanine (G). The figure shows the evolutionary pathway going from a GNC code (4 codons) through a SNS code (16 codons), to the Universal Genetic Code. To decode a codon, the first letter is matched in the left column, the second letter on the top row, and the third letter on the right column. The 64 codons, along with the amino acid or stop signal they specify, are shown in the boxes. All but two of the amino acids (methionine and tryptophan) have more than one codon. Note that in the mRNA, uracil replaces the thymine found in DNA. A (after Di Giulio 2008), B (after Ikehara 2002); C, the Universal Genetic Code. Instead of a conventional representation, the modern genetic code is shown reflecting the order of codon occurrence from GNC, to SNS, to a modern code (columns G and U inverted) (modified from Mace and Gillet 2016).

Table 2. Universal Genetic Code and its method of conversion to numerical codons.

Table 3. Universal Genetic code showing numerical codons.

Table 4. 20 Primary Amino Acids in the Genetic Code and their corresponding numerical codons.

Table 5. Conversion of numerical codons into corresponding amino acids and vice versa using CATI software. 
Supplementary Materials for

\section{The Origin of Information System in the RNA/Protein World: Simulation Model of the Evolution of Translation and the Genetic Code}

By Sankar Chatterjee* and Surya Yadav

*Correspondence to: sankar.chatterjee@ttu.edu

Appendix A

This appendix is a user guide for the visualization model developed in the AnyLogic software. It describes as to how to run the visualization model for the each stage of the translation machines. The visualization models are hosted on the AnyLogic cloud, a service that allows simulation models to be available online on Internet. These hosted models can be run under a browser such as Chrome, Internet Explorer, and Fire Fox, etc. In the following sections, it is assumed that an Internet browser is up and running either on a laptop or on a PC.

Instructions for Stage I Visualization

This section shows the steps to run the stage I model. Please follow these steps:

Step 1. Paste the following URL link in your browser's address line and go to that link:

https://cloud.anylogic.com/model/5a297e73-af36-4af5-a92c$\underline{\text { a416021a9bda? mode }=\text { DASHBOARD\&experiment }=82 \mathrm{e} 7 \mathrm{de} 3 \mathrm{a}-\mathrm{b} 6 \mathrm{df}-4 \mathrm{ab} 2-9304-e e b a 402 \mathrm{cc} 447}$

Step 2. You should see a web page that looks like the one shown in figure A1. 


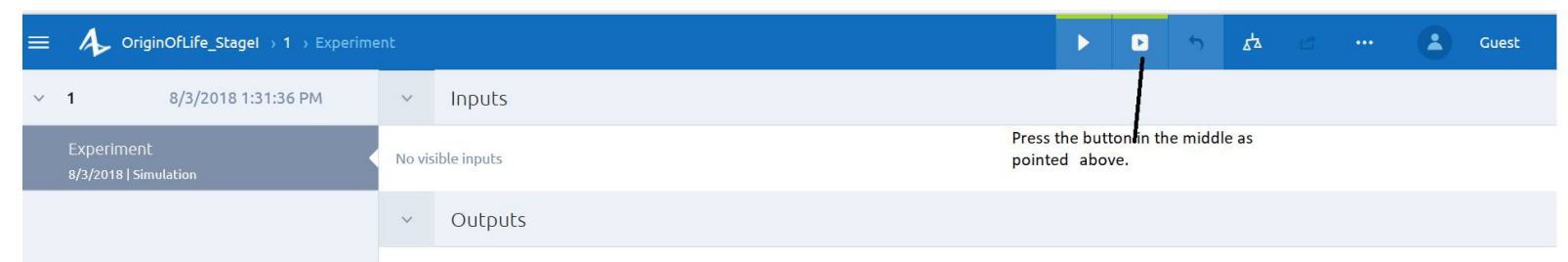

Figure A1. A screen shot of the web page showing the button to be pushed.

Step 3. Press the button as indicated in figure A1. The visualization model should start running in a few seconds.

Step 4 . The model can be stopped any time by closing the webpage.

\section{Instructions for Stage II Visualization}

This section shows the steps to run the stage II model. Please follow these steps:

Step 1. Paste the following URL link in your browser's address line and go to that link:

https://cloud.anylogic.com/model/c19bfa43-d338-4a1b-93c34071d738add7?mode $=$ DASHBOARD

Please follow the steps 2 to 4 as described above in the stage I visualization.

Instructions for Stage III Visualization

This section shows the steps to run the stage III model. Please follow these steps:

Step 1. Paste the following URL link in your browser's address line and go to that link: 
Please follow the steps 2 to 4 as described above in the stage I visualization.

\section{Note:}

Please note that the visualization models are hosted in AnyLogic Cloud. After running the model, the screen can be closed by clicking on the " $X$ " button on top right-hand side. These models have an 'Animated Run Time Limit' in the cloud. The model can be closed and re-run if you get a message as shown below:

"Animated run time limit

The model has run for the maximum time allowed..."

Simply close the message box, then close the model by pressing on the "X" button and run the model by pressing on the play button as shown in Stage I instructions. 